Programa de Doctorado de Inteligencia Artificial Escuela Técnica Superior de Ingenieros Informáticos

PhD Thesis

\title{
Týr: Storage-Based HPC and Big Data Convergence Using Transactional Blobs
}

\author{
Author: Pierre Matri \\ Supervisors: Prof. Dra. María S. Pérez Hernández \\ Prof. Dr. Gabriel Antoniu
}


Tribunal nombrado por el Sr. Rector Magfco. de la Universidad Politécnica de Madrid, el día 11 de Junio de 2018.
Presidente: Dr. Antonio Pérez Ambite
Vocal: $\quad$ Dr. Jesús Carretero Pérez
Vocal: $\quad$ Dr. Antonio Cortés Roselló
Vocal: $\quad$ Dr. Christian Pérez
Secretario: Dr. Alberto Sánchez Campos
Suplente: Dr. Antonio García Dopico
Suplente: Dr. Alejandro Calderón Mateos

Realizado el acto de defensa y lectura de la Tesis el día 11 de Junio de 2018 en la Escuela Técnica Superior de Ingenieros Informáticos

Calificación:

EL PRESIDENTE

VOCAL 1

VOCAL 2

VOCAL 3

EL SECRETARIO 
To Dimitris.

To my family, who make it all worthwile. 


\section{Agradecimientos}

My very first comments will be directed to my three tremendous advisors, María, Alexandru and Gabriel. You have set an example of excellence as researchers, mentors, instructors, and tactful moderators of my wildest ideas. It is needless to say that this thesis would not have been possible without your continuous support, guidance and wisdom.

I could not thank enough my fellow Ph.D students from the BigStorage project. I cannot express how greatful I am for having been awesome colleagues, and above all awesome friends. Thank you all. Álvaro, Danilo, Dimitris, Eugene, Georgios, Fotis(es), Linh, Michał, Nafiseh, Ovidiu, Thanos, Umar, Yacine, you all helped in your own way making these three years an unforgettable experience, hence significantly contributing to this thesis.

Many thanks to the members of the Ontology Engineering Group, who were here to welcome me, share support, bring tasty cakes, and force me to learn Spanish all along the way. For all this, thank you Ahmad, Daniel, Freddy, Idafen, Julia, María, Miguel Ángel, Nelson, Pablo, Raúl, and everybody I don't have the space to name. A special thanks goes to Jose Ángel and Ana for helping me through the endless admistrative dedalus.

Similar comments goes to the tremendous KerData team I first started research with in Rennes, and with whom I finish writing this manuscript. Luis, Hadi, Nathanaël, Matthieu, Radu, Luc. You did definitely contribute to this thesis, all in your own way, around lunches, coffee and team meetings. I hereby devise my beloved coffee machine to the team. Thanks to Aurélie for her administrative support that prevented precocious hair loss.

I want to also thanks Rob Ross and Phil Carns for warmly welcoming me at Argonne National Laboratory during three summer months. Besides being a fantastic research environment, I could also share precious time with awesome people I simply cannot forget acknowledging in here.

Let me switch to french for one paragraph. Je tiens bien évidemment à remercier ma famille, pour leur soutien inconditionnel qui m'a toujours 


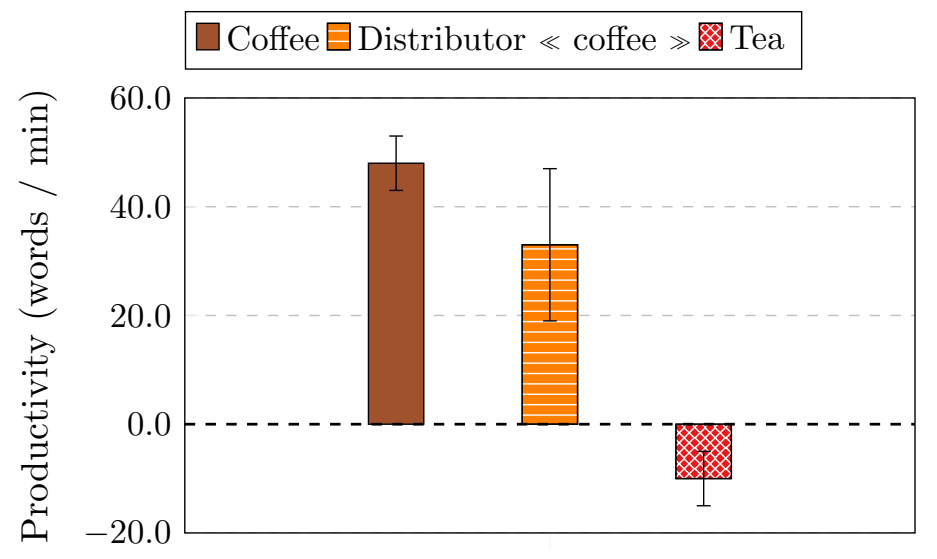

Figure

Empirically-

determined pro-

ductivity estimation relative to the available coffee supply around the office, with $95 \%$ confidence intervals.

permis de me consacrer à la réalisation de mes rêves. Parce-que même au loin vous êtes toujours là, vous me supportez depuis toujours, et vous avez toujours cru en moi. Merci maman, papa. Merci Céline, François et Caroline. Merci Valéry, Marie et Arthur. Merci Sarah. Merci Gabriel.

I need to dedicate a paragraph for all those who willingly or unwillingly contributed to this thesis. In no particular order, thanks to the reviewers of VLDB, CCGrid and Cluster that, by rejecting my contributions, led me to seek for the better. Thanks to Iberia, whose incalculable delays developed my ninja writing skills in airports around the world. Thanks to all the worldwide coffee supply chain, whose hard work and effective caffeine supported my productivity all along the way, as plotted in Figure 1.

I want to finish with a very special mention for Dimitris, who left us way too soon, way too young, and to whom I dedicate this thesis. Dimitris, I could not thank you enough for being one of the most inspiring and cheerful souls I have ever known. I could not thank you enough for your courage and inspiration. I could not thank you enough for the laughter and the beers. I could not thank you enough for being you. I simply could not thank you enough. May you rest in peace, with all my love, my wishes and my thoughts. 


\begin{abstract}
The increasingly growing data sets processed on HPC platforms raise major challenges for the underlying storage layer. A promising alternative to traditional file-based storage systems are simpler blobs (binary large objects). They offer lower overhead and better performance at the cost of largely unused features such as file hierarchies or permissions. In a similar fashion, blobs are increasingly considered for replacing distributed file systems for Big Data Analytics (BDA) or as a base for storage abstractions like keyvalue stores or time-series databases. From these observations we advocate that blobs provide a solid storage model for convergence between HPC and BDA platforms. We identify data consistency as a hard problem to solve in this context because of the different choices made by both communities: while BDA developers typically rely on the storage system to provide data access coordination, the lack of such semantics on HPC platforms requires developers to use application-level tools for this task. In this thesis we propose the key design principles of Týr, a converging storage system designed to answer the needs of both HPC and BDA applications, natively offering data access coordination in the form of transactions. We demonstrate the relevance and efficiency of its design in the light of convergence in multiple applicative contexts from both communities. These experiments validate that Týr delivers its promise of high-throughput and versatility, hence fueling storage-based convergence between HPC and BDA.
\end{abstract}




\section{Resumen}

La creciente cantidad de datos procesados en plataformas HPC supone un reto para el sistema de almacenamiento subyacente. Una alternativa prometedora a los sistemas de almacenamiento basado en ficheros es el uso de BLOBs (Binary Large OBjects). Esta alternativa ofrece menor sobrecarga y mejor rendimiento a cambio de eliminar características típicas de los sistemas de ficheros, como la jerarquía en forma de directorios o los permisos. De manera análoga, los blobs pueden utilizarse para reemplazar sistemas de ficheros en el área de Big Data Analytics (BDA) o como base para otras abstracciones de almacenamiento, tales como bases de datos clave-valor o de series de tiempo. A partir de estas observaciones, podemos concluir que los blobs son un modelo de almacenamiento sólido para lograr la convergencia entre plataformas HPC y BDA. En este contexto, uno de los problemas críticos que hay que resolver es la consistencia de los datos, debido a los diferentes elecciones de cada una de las comunidades: mientras que los desarrolladores de BDA delegan habitualmente la responsabilidad de coordinar el acceso a los datos al sistema de almacenamiento, la falta de dicha capacidad en las plataformas HPC requiere que los desarrolladores tengan que utilizar herramientas a nivel de aplicación para realizar esta tarea. Esta tesis propone los principios de diseño principales de Týr, un sistema de almacenamiento convergente diseñado para responder a las necesidades de aplicaciones HPC y BDA, ofreciendo de forma nativa la coordinación en el acceso a los datos en forma de transacciones. La tesis demuestra la relevancia y eficiencia de este diseño aplicado a múltiples escenarios de ambos campos. Los experimentos implementados muestran las características de rendimiento y versatilidad ofrecidas por Týr, lo que supone un importante impulso para lograr la deseada convergencia entre HPC y BDA. 


\section{Contents}

1 Introduction 1

1.1 Objectives ............................

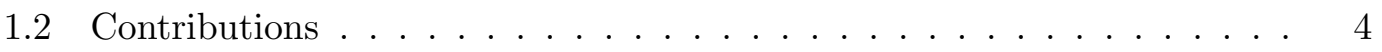

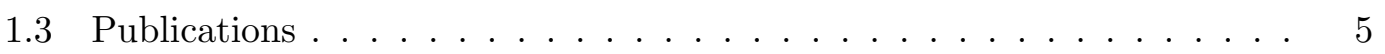

1.4 Organization of the manuscript $\ldots \ldots \ldots \ldots \ldots$

I Context: distributed storage, consistency for HPC and Big Data 9

2 HPC and BDA: Divergent stacks, convergent storage needs 11

2.1 Comparative overview of HPC and BDA stacks . . . . . . . . . . 12

2.2 HPC: Centralized, file-based storage . . . . . . . . . . . 14

2.3 BDA: Modular, application-purpose storage . . . . . . . . . . 15

2.4 Conclusions: challenges of storage convergence between HPC and BDA 16

3 Distributed storage paradigms

3.1 Distributed file systems . . . . . . . . . . . . . . 18

3.2 Key-value stores $\ldots \ldots \ldots \ldots \ldots \ldots$

3.3 Document, columnar, graph databases . . . . . . . . . . 24 24

3.4 Blob storage . . . . . . . . . . . . . . . . . . . . 27

3.5 Conclusions: which storage paradigm for converging storage? . . . . . 30

4 Consistency management 31

4.1 Storage consistency models . . . . . . . . . . . . . . . . 32

4.2 Application-specific consistency management . . . . . . . . . . . 34

4.3 Transactional semantics . . . . . . . . . . . . . . . 36 
4.4 CAP: Consistency, Availability, Partition tolerance . . . . . . . . . 40

4.5 Conclusions: which consistency model for converging storage? . . . . . . 41

II Týr: a transactional, scalable data storage system 43

5 Blobs as storage model for convergence

5.1 General overview, intuition and methodology . . . . . . . . . . . . 45

5.2 Storage call distribution for HPC and BDA applications . . . . . . . . 49

5.3 Replacing file-based by blob-based storage . . . . . . . . . . 52

5.4 Conclusion: blob is the right storage paradigm for convergence . . . . 58

6 Týr design principles and architecture 61

6.1 Key design principles . . . . . . . . . . . . . . . . . . 62 62

6.2 Predictable data distribution . . . . . . . . . . . . 63

6.3 Transparent multi-version concurrency control . . . . . . . . . . . . . 64 64

6.4 ACID transactional semantics . . . . . . . . . . . . . . . . . 66 66

6.5 Atomic transform operations . . . . . . . . . . . . . . 67

7 Týr protocols

7.1 Lightweight transaction protocol . . . . . . . . . . . . 72

7.2 Handling reads: direct, multi-chunk and transactional protocols . . . . 74

7.3 Handling writes: transactional protocol, atomic transforms . . . . . . 78

7.4 Bookkeeping: purging expired versions . . . . . . . . . . . . . 80 80

8 Implementation details 83

8.1 Multiple, configurable keyspaces . . . . . . . . . . . . . . 84

8.2 Persistent data storage . . . . . . . . . . . . . . . 85

8.3 Fault-tolerance . . . . . . . . . . . . . . . . 85

8.4 Event-driven server design . . . . . . . . . . . . . . . 88

III Evaluation: Týr in support of HPC and BDA convergence 91

9 Real-time, transactional data aggregation in support of system monitoring for BDA 93

9.1 ALICE and MonALISA . . . . . . . . . . . . . . . . 94 
9.2 Experimental setup . . . . . . . . . . . . . . . . . 95

9.3 Transactional write performance $\ldots \ldots \ldots$. . . . . . . . . 96

9.4 Read performance . . . . . . . . . . . . . . . . . 99

9.5 Reader / writer isolation . . . . . . . . . . . . . . . . . . 100

9.6 Horizontal scalability . . . . . . . . . . . . . . . . . . . . . 101

9.7 Conclusions . . . . . . . . . . . . . . . . . . . . . . 101

10 Large-scale logging in support of computational steering for HPC 103

10.1 Distributed logging for computational steering . . . . . . . . . . . 104

10.2 Experimental setup . . . . . . . . . . . . . . . . . 106

10.3 Baseline performance . . . . . . . . . . . . . . . . . . . 109

10.4 Further increasing append performance by increasing parallelism . . . . 110

10.5 Scaling distributed logging up to 120,000 cores . . . . . . . . . . . 112

10.6 Conclusions . . . . . . . . . . . . . . . . . . . . . 113

11 Týr for HPC and BDA convergence 115

11.1 Týr as a storage backend for HPC applications . . . . . . . . . . . 115

11.2 Týr as a storage backend for BDA applications . . . . . . . . . . . . 119

11.3 Conclusions . . . . . . . . . . . . . . . . . . . . . 120

12 TýrFS: Building a file system abstraction atop Týr 123

12.1 Motivation: the overhead of reading small files . . . . . . . . . . 124

12.2 TýrFS design . . . . . . . . . . . . . . . . . . . . . 126

12.3 TýrFS implementation . . . . . . . . . . . . . . . . . 130

12.4 Experimental setup . . . . . . . . . . . . . . . . . . . 134

12.5 Performance in support of HPC applications . . . . . . . . . . . . 135

12.6 Performance in support of BDA applications . . . . . . . . . . 137

12.7 Conclusions . . . . . . . . . . . . . . . . . . . . . . 140

IV Conclusions: Achievements and Perspectives 143

13 Conclusions

13.1 Achievements . . . . . . . . . . . . . . . . . . 146

13.2 Perspectives . . . . . . . . . . . . . . . . . . . . 148 


\section{List of Figures}

1 Productivity estimation relative to the available coffee supply around the office. . . . . . . . . . . . . . . . . . . . . viii

2.1 Typical HPC and BDA software stacks. . . . . . . . . . . . . 12

5.1 Measured relative amount of different storage calls to the persistent file system for HPC applications . . . . . . . . . . . . . . 5 50

5.2 Measured relative amount of different storage calls to the persistent file system for BDA applications . . . . . . . . . . . . 51

5.3 Average agreggate throughput across all HPC applications varying the compute-to-storage ratio, with $95 \%$ confidence intervals. . . . . . . . . 54

5.4 Comparison of read throughput for each HPC application with Lustre, BlobSeer and Rados, with $95 \%$ confidence intervals. . . . . . . . . 54

5.5 Comparison of write throughput for each HPC application with Lustre, BlobSeer, and Rados, with 95\% confidence. . . . . . . . . . . 55

5.6 Average performance improvement relative to Lustre for HPC applications using blob-based storage, with $95 \%$ confidence. . . . . . . . 55

5.7 Comparison of read throughput for each Big Data application with HDFS, BlobSeer, Rados and CephFS, with 95\% confidence intervals. . . . . . 58

5.8 Comparison of write throughput for each Big Data application with HDFS, BlobSeer, Rados and CephFS, with 95\% confidence intervals. . . 58

5.9 Average performance improvement relative to HDFS for Big Data applications using blob-based storage, with $95 \%$ confidence intervals. . . . . . 59

6.1 Týr versioning model . . . . . . . . . . . . . . . . . . . . 65

6.2 Týr version management example . . . . . . . . . . . . . 65 65 
7.1 Warp lightweight transaction protocol overview. In this example, the directionality of the edge $\left(t_{1}, t_{2}\right)$ will be decided by $s_{4}$, last common server in the transaction chains, during the backwards pass. Similarly, the directionality of $\left(t_{2}, t_{3}\right)$ will be decided by $s_{4} \ldots \ldots \ldots \ldots$

7.2 Týr read protocol for a read spanning multiple chunks. The client sends a read query for chunks $c_{1}$ and $c_{2}$ to the version manager, which relays the query to the servers holding the chunks with the correct version information. . . . . . . . . . . . . . . . 76

7.3 Týr read protocol inside a transaction. The client sends a read query for chunks $c_{1}$ and $c_{2}$ to the version manager, which relays the query to the servers holding the chunks with the correct version information, and responds to the client with a snapshot of the latest chunk versions. Subsequent read on $c_{3}$ is addressed directly to the server holding the chunk data using the aforementioned chunk version information. . . . . 777

8.1 High-level internal architecture of Týr server . . . . . . . . . . 84

9.1 Simplified MonALISA data storage layout, showing five generators on two different clusters, and three levels of aggregation. Solid arrows indicate events written, dotted arrows represent event aggregation. Each rectangle indicates a different blob. Dotted rectangles denotes aggregate blobs. . . . . . . . . . . . . . . . . . 95

9.2 Baseline performance of Týr, Rados, BlobSeer and Azure Storage, varying the number of clients, with $95 \%$ confidence intervals. . . . . . . . 97

9.3 Synchronized write performance of Týr, Rados, BlobSeer and Azure Storage, varying the number of clients, with $95 \%$ confidence intervals. . 98

9.4 Read performance of Týr, Rados, BlobSeer and Azure Storage, varying the number of clients, with $95 \%$ confidence intervals. . . . . . . . . . 99

9.5 Read throughput of Týr, Rados, BlobSeer and Azure Storage for workloads with varying read to write ratio. Each bar represents the average read throughput of 200 concurrent clients averaged over one minute. . . 100

9.6 Týr horizontal scalability. Each point shows the average throughput of the cluster over a one-minute window with a $65 \%$ read / $35 \%$ write workload, and $95 \%$ confidence intervals. . . . . . . . . . . . . . 101 
10.1 Illustration of telemetry event collection. Simulation ranks generate events to a central log (a). Such events are used directly for online tasks such as visualization or computational steering (b), or are stored for further offline processing (c). Computational steering in turn controls the simulation $(\mathrm{d}) \ldots \ldots \ldots \ldots$. . . . . . . . . . . . . . . . . . . . . .

10.2 Append performance of Lustre and GPFS on Theta to a single file, varying the number of clients. The performance axis uses a logarithmic scale. 108

10.3 Append log architecture using write proxys for parallel file systems. . . . 109

10.4 Baseline append performance, using up to 8,192 append client cores, with $1 \mathrm{~KB}$ appends. . . . . . . . . . . . . . . . . . . . . . . 110

10.5 Illustration of the multiple independent files method, where each output $\log$ is independently mapped to a group of nodes. . . . . . . . . . . . 1111

10.6 Baseline append performance, using up to 8,192 append client cores, with $1 \mathrm{~KB}$ appends. . . . . . . . . . . . . . . . . . . . . 112

10.7 Aggregate append performance of Týr at large scale using up to 100,000 writer and 20,000 storage cores. . . . . . . . . . . . . . . 113

11.1 Comparison of read throughput for each HPC application with Lustre, BlobSeer and Rados, with $95 \%$ confidence intervals. . . . . . . . . . . 116

11.2 Comparison of write throughput for each HPC application with Lustre, BlobSeer, and Rados, with $95 \%$ confidence. . . . . . . . . . . . . . . 117

11.3 Average performance improvement relative to Lustre for HPC applications using blob-based storage, with $95 \%$ confidence. . . . . . . . . . 117

11.4 Average performance improvement relative to Lustre for HPC applications using blob storage, with $95 \%$ confidence on Theta. . . . . . . . . 118

11.5 Average performance improvement at scale relative to 32 nodes setup for HPC applications using blob-based storage on Theta. . . . . . . . . . 119

11.6 Comparison of read throughput for each Big Data application with HDFS, BlobSeer, Týr, Rados and CephFS, with 95\% confidence intervals. . . . 119

11.7 Comparison of write throughput for each Big Data application with HDFS, BlobSeer, Týr, Rados and CephFS, with 95\% confidence intervals.120

11.8 Average performance improvement relative to HDFS for Big Data applications using blob-based storage, with $95 \%$ confidence intervals. . . . . . 121 
12.1 Lustre and OrangeFS read throughput for a fixed-size data set when varying the size of the individual files it is composed of. . . . . . . . . 125

12.2 Breakdown of reads between metadata (M) and data (D) access for a fixed-size data set varying the size of the individual files it is composed of.125

12.3 Typical read protocol for a small file with centralized metadata management (e.g. Lustre or HDFS). . . . . . . . . . . . . . . 126

12.4 Typical read protocol for a small file with decentralized metadata management on three storage nodes $s_{1}, s_{2}$ and $s_{3}$ (e.g. OrangeFS). . . . 127

12.5 High-level overview of the proposed TýrFS architecture. Each node integrates both a data and a metadata service. The metadata service itself is composed of a local metadata store and the dynamic metadata replication service. . . . . . . . . . . . . . . . . . 130

12.6 TýrFS, Lustre and OrangeFS read throughput for a fixed-size data set, varying the size of the individual files it is composed of. . . . . . . . . 136

12.7 Breakdown of reads between metadata (M) and data (D) access for a fixed-size data set varying the size of the individual files it is composed of.136

12.8 TýrFS horizontal scalability, varying the number of clients and the number of cluster nodes with $32 \mathrm{~KB}$ input files. . . . . . . . . . . . . 137

12.9 TýrFS, Ceph and HDFS read throughput for a fixed-size data set, varying the size of the individual files it is composed of. . . . . . . . . . . 138

12.10TýrFS, Ceph and HDFS write throughput with Sort (balanced I/O) and Tokenizer (write-intensive) applications. . . . . . . . . . . . . . 139

12.11Application completion time difference with $32 \mathrm{MB}$ input files relative to that obtained with $32 \mathrm{~KB}$ input files, with TýrFS, Ceph and HDFS. $100 \%$ represents the baseline performance for 32 MB files. . . . . . . . . 140 


\section{List of Tables}

5.1 HPC and BDA application summary . . . . . . . . . . 49

5.2 Spark directory operation breakdown. . . . . . . . . . . . . 51

5.3 HPC storage call translation rules on a flat namespace . . . . . . . 53

5.4 BDA storage call translation rules on a flat namespace . . . . . . . 57 


\section{List of Algorithms}

1 High-level algorithm of the metadata resolver. . . . . . . . . . . . . 133 


\section{Chapter 1}

\section{Introduction}

Among the major trends in computer science are the growth of High-Performance Computing (HPC) and the Big Data phenomenon. Both lead to a dramatic increase of the size, sophistication and complexity of HPC and Cloud platforms. One of the challenges associated with this movement is data storage and management, which is widely recognized as critical to the overall performance of a broad class of applications. This challenge is increasingly relevant as the data size and throughput requirements of largescale applications grow. Both HPC and Big Data Analytics (BDA) communities have paved the way to significant increases in storage performance by optimizing existing systems or inventing new data models able to cope with this new demand. Yet, the astonishing growth rate of current extreme scale platforms and the predicted storage requirements of tomorrow's systems make the quest for increased performance more critical than ever.

Researchers in the fields of distributed computing and data storage are spending considerable resources to tackle these issues. Interestingly, while the challenges are similar between cloud computing and extreme computing, both communities have diverged significantly in terms of proposed solutions and research orientation. As a result, HPC and BDA stacks remain completely separated today. Indeed, cloud computing platforms exhibit desirable features such as scalability, elasticity and usability, which represents for its users a cost effective platform for large-scale data warehousing and analytics. The gap between both platforms tends to reduce thanks to the addition of HPC-oriented features such as GPU computing or high-speed interconnects in new cloud computing platforms (118). However, running HPC applications on this type of 
platforms remains difficult due to the high overhead of cloud techniques such as virtualization, or because of the lower performance and higher latency of the interconnection between compute nodes (18). Although these challenges are the focus of an abundant scientific literature, the divergences remain significant.

On the contrary, while little studied in practice, the research orientations for the data management layer for HPC and BDA are similar: trading versatility for performance. As such, we advocate that storage offers a high potential for convergence despite its current divergences. Indeed, one side, extreme computing relies on optimizing existing file systems such as Lustre (142), GPFS (140) or PVFS (137). One of the main rationales behind this choice is the required support for legacy applications, some of which being close to 3 decades old (62). On the other side, the fast-evolving stack adopted by the cloud community embraces and combines small, workload-specific storage systems that achieve high performance by dropping a significant portion of distributed file systems semantics.

Yet, we witness in the last years a trend to apply strikingly similar techniques in support of these divergent orientations. In particular, object storage has proven to be a strong storage model both for extreme and cloud computing. In the former, it serves as support for Lustre (32) or DeltaFS (175) while in the latter it is the base building block for the Ceph (167) file system, or is exposed directly to the user in the form of key-value or document stores (45; 55; $92 ;$, 148).

Despite this similarity in approaches leveraged, several critical divergences remain between the two communities. Among the most important ones are the choices made with respect to storage consistency. This can include synchronizing storage operations or ensuring that the storage system remains in a consistent state in case of failures, and is critical for a range of applications that include real-time indexing or data aggregation systems. While the HPC community tends to control such consistency at the application level, the Big Data community typically delegates this task to the storage system through the use of storage transactions (70). Transactions essentially group multiple storage operations as a single task that is applied consistently and atomically to the storage system. Yet, no blob storage system today offer transaction support, which is difficult due to the challenges associated with the potentially large size of the objects. 


\section{$1.1 \quad$ Objectives}

In this thesis, we hypothesize that despite important divergences, storagebased convergence between HPC and big data is not only possible, but also leads to substantial performance improvements over the state of the

art. Considering the common trend towards object storage in both HPC and BDA communities, we assert that such convergence can be achieved through a variety of use-cases while retaining support for legacy applications.

In order to prove this hypothesis, this thesis fulfills a series of sub-objectives summarized as follows:

1. Investigate a series of existing data storage paradigms for distributed computing and to understand their limitations in respect to HPC and Big Data convergence,

2. Detail the main approaches to data consistency as leveraged in both communities, understand their limitations and respective applicability for convergence,

3. Determine through empirical evaluation whether such convergence is possible by studying the storage I/O patterns performed by a series of I/O-intensive applications from both HPC and Big Data communities,

4. Formulate a series of design principles that lay the foundation of an efficient data storage system aimed at HPC and Big Data convergence,

5. Formally describe the protocols and algorithms that can be leveraged by a storage system based on these design principles,

6. Provide a prototype implementation of a storage system based on the aforementioned design principles, protocols and algorithms,

7. Evaluate this prototype in multiple contexts that factually prove its relevance for both HPC and Big Data platforms with real-life use-cases and applications, while retaining compatibility with legacy applications. 


\subsection{Contributions}

The main contributions of this thesis can be summarized as follows:

An empirical proof that object-based storage is suitable for achieving storagebased convergence between HPC and BDA. We leverage a series of real-world applications from the HPC community as well as various industry-standard benchmarks from the Big Data community and analyze the distribution of their storage calls. We conclude that the vast majority of the storage calls performed by these applications are simple file reads and writes that are easily covered by object storage systems. We also demonstrate that the rest of the calls can be mapped atop object storage. In this case, we prove that the performance decrease caused by such mapping is largely compensated by the performance gains permitted by the use of a storage system highly optimized for simple object reads and writes. This work was carried out in collaboration with Yevhen Alforov, Álvaro Brandon, Michael Kuhn, Philip Carns, Thomas Ludwig, Alexandru Costan, María S. Pérez and Gabriel Antoniu. It was published in (110).

Týr: a transactional storage system aimed at storage-based convergence between HPC and Big Data. We propose a series of design principles for building a converging storage system based on the observations mentioned in the previous paragraph. In particular, we defend that this system should provide transactions as a way to enforce strong consistency at the storage level in order to offer support for a variety of use-cases such as real-time data aggregation. We describe the algorithms and protocols that enable it to guarantee such consistency at high speed. Based on this design we introduce Týr, a transactional file system built with convergence in mind. We present the techniques we used to convert these contributions to a practical system that we evaluate extensively using the applications mentioned in the previous paragraph. This work was carried out in collaboration with Alexandru Costan, Gabriel Antoniu, Jesús Montes and María S. Pérez. It was published in (113; 114).

A Týr-based distributed logging architecture for HPC platforms. We leverage Týr transactional capabilities at high velocity to offer fast and scalable distributed logging for HPC platforms. We prove on up to 120,000 cores of a leadership-class supercomputer that this architecture enables computational steering services to scale 
well beyond what was possible using file-based storage. We additionally demonstrate how transactions could be used in this context to provide efficient real-time monitoring. This work was carried out in collaboration with Philip Carns and Robert Ross at Argonne National Laboratory, Chicago, Illinois, USA, as well as María S. Pérez, Alexandru Costan and Gabriel Antoniu. It was published in (111).

\title{
A Týr-based, large-scale system monitoring and data aggregation service for
} BDA. We build a storage backend for the MonALISA monitoring service (101; 102) of the CERN LHC Alice experiment (14). This backend enables MonALISA to scale beyond what was previously possible with the previous relational data storage. It leverages Týr built-in transactions to perform data aggregation in near real-time at high speed. This work was carried out in collaboration with Alexandru Costan, Gabriel Antoniu, Jesús Montes and María S. Pérez. It was published in (113; 114).

\begin{abstract}
A POSIX-compliant, transactional, distributed file system implementation built atop Týr. We detail the design and implementation of a complete, POSIXcompliant distributed file system built as a thin layer atop Týr. By doing so, we prove that Týr effectively retains compatibility with legacy applications that expect such filebased storage to be provided. This file system leverages dynamic metadata replication to provide fast access to files, that we prove to benefit significantly to applications processing large amounts of small files. We demonstrate that this file system performs well and shows near-linear scalability properties on both HPC and Big Data platforms. This work was carried out in collaboration with María S. Pérez, Alexandru Costan, Luc Bougé and Gabriel Antoniu. TýrFS was published in (115); its underlying metadata management principles were published in $(112$; 116$)$.
\end{abstract}

\subsection{Publications}

The work presented in this manuscript was published in several peer-reviewed journals, conferences and research reports listed below:

\section{Journal articles}

- Matri, P., Pérez, M. S., Costan, A., Bougé, L., and Antoniu, G. Keeping up with storage: Decentralized, write-enabled dynamic geo-replication. Future 
Generation Computer Systems (2017) - JCR Q1 (116)

\section{Conferences}

- Matri, P., Costan, A., Antoniu, G., Montes, J., And Pérez, M. S. Towards efficient location and placement of dynamic replicas for geo-distributed data stores. In Proceedings of the ACM 7th Workshop on Scientific Cloud Computing (2016), ACM, pp. 3-9 (112)

- Matri, P., Costan, A., Antoniu, G., Montes, J., and Pérez, M. S. Týr: blob storage meets built-in transactions. In High Performance Computing, Networking, Storage and Analysis, SC16: International Conference for (2016), IEEE, pp. 573-584 - CORE A, Best student paper award finalist (113)

- Matri, P., Alforov, Y., Brandon, A., Kuhn, M., Carns, P., and Ludwig, T. Could blobs fuel storage-based convergence between HPC and Big Data? In Cluster Computing (Cluster), 2017 IEEE International Conference on (2017), IEEE, pp. 81-86 - CORE A (110)

- Matri, P., Costan, A., Antoniu, G., and Pérez, M. S. TýrFs: Increasing small files access performance with dynamic metadata replication. In Cluster, Cloud and Grid Computing (CCGrid), IEEE/ACM 18th International Symposium on (2018), IEEE - CORE A (115)

- Matri, P., Carns, P., Ross, R., Costan, A., Pérez, M. S., and Antoniu, G. SLoG: A large-scale logging middleware for HPC and Big Data convergence. In Distributed Computing Systems (ICDCS), 2018 IEEE 38th International Conference on (2018), IEEE - CORE A (111)

\section{Technical reports}

- Matri, P., Costan, A., Antoniu, G., Montes, J., and Pérez, M. S. Týr: Efficient Transactional Storage for Data-Intensive Applications. Technical Report RT-0473, Inria Rennes Bretagne-Atlantique ; Universidad Politécnica de Madrid, Jan. 2016 (114) 


\subsection{Organization of the manuscript}

The rest of the manuscript is organized in four parts:

The first part details the context of our work, presenting the state of the art in related research areas. It consists of three chapters. Chapter 2 provides an overview of the applicative stacks for BDA and HPC. It identifies the key divergences as well as the convergence opportunities, including the storage layer. Chapter 3 zooms on that layer, describing the diverse storage paradigms that are common in BDA and HPC platforms. We describe their applicability for storage-based convergence and the related challenges in this context. In Chapter 4 we focus on one of these challenges: data consistency and write coordination. We review the different storage consistency models and semantics in the current scientific literature in the light of HPC and BDA convergence. We conclude with the limitations of the existing approaches and the new challenges that need to be tackled for such convergence to meet the ever-growing needs of large-scale data analytics and simulation applications.

The second part motivates the choice of blobs as a storage model to address the challenges highlighted in the first part. Based on this choice, it introduces the core contribution of this thesis: Týr, a transactional blob storage system aimed at supporting HPC and BDA use-cases at large scale. It consists of four chapters. Chapter 5 details the main reasons making blobs an ideal storage model candidate for HPC and BDA convergence. We leverage a series of preliminary experiments that prove that the data model and API provided by blob storage systems cover the needs of a series of representative applications from both communities. We show with two state-ofthe-art blob storage systems from the scientific literature the performance benefits compared to file-based storage. Chapter 6 builds from these observations a set of key design principles that drive the design of Týr. These principles answer at the same time the needs of legacy applications while considering the additional challenges of convergence identified in the first part. Chapter 7 details the protocols implementing these principles. In particular, we present the read protocols able to guarantee read consistency for large objects at high velocity, the lightweight transaction protocol which forms the base of Týr as well as the bookkeeping protocol for managing expired data at scale. Finally, Chapter 8 discusses the key engineering aspects we follow when 
developing a software artifact implementing the aforementioned design principles and protocols.

The third part evaluates the relevance of Týr for HPC and BDA convergence in various applicative contexts. It contains four chapters. First, Chapter 9 details the BDA-oriented storage requirements of a real-life monitoring platform for a large-scale experiment from the CERN. In particular, it motivates the pertinence of transactions for real-time data aggregation and experimentally shows how the design of Týr provides an efficient storage solution for this application. Chapter 10 details how the design of Týr is relevant to large-scale data logging in the context of computational steering on HPC platforms. We highlight the limitations of the current solutions proposed in this context, and demonstrate on a leadership-class supercomputer that Týr provides an efficient answer to these challenges at very large scale. Chapter 11 demonstrates using the same applications as in Chapter 5 that the design of Týr effectively delivers its promises for existing applications. Finally, we present in Chapter 12 the design of TýrFS, a lightweight file-based interface implemented atop Týr that permits retaining support for legacy applications expecting file system semantics. We show how such interface can be optimized for the unique challenges of managing large numbers of small files.

The fourth part wraps up our work. Chapter 13 summarizes the aforementioned contributions, discusses the limitations of our work and presents a series of perspectives that are interesting to explore in the context of HPC and BDA convergence. 


\section{Part I}

\section{Context: distributed storage, consistency for HPC and Big \\ Data}





\section{Chapter 2}

\section{HPC and BDA: Divergent stacks, convergent storage needs}

In the decades since the invention of the computer, HPC has contributed significantly to our quality of life - driving scientific innovation, enhancing engineering design and consumer goods manufacturing, as well as strengthening national and international security. This has been recognized and emphasized by both government and industry, with major ongoing investments in areas encompassing weather forecasting, scientific research and development as well as drug design and healthcare outcomes. More recently, with the explosion of data available from the development of the internet, industry and other organizations have recognized that data represents a gold mine of competitive possibility. Companies like Google, Uber, Airbnb and Facebook may be the best known users of BDA, but at the same time numerous traditional organizations from advertising to weather forecasting have started to look at BDA solutions as they try to find new ways to interact with customers and create new business models and opportunities.

HPC applications are increasingly facing very large amounts of data as well. The key difference to be seen in that context between BDA and HPC applications is the type of data both systems are dealing with. While the focus of HPC is mainly on structured data, BDA often need to handle new types of data originating from a wide variety of sources, with very diverse structures if any. Hence the challenges associated with the processing of data is mainly a software challenge. Furthermore, most tasks, whether they are deemed HPC or BDA, are part of a wider workflow. For example, to 

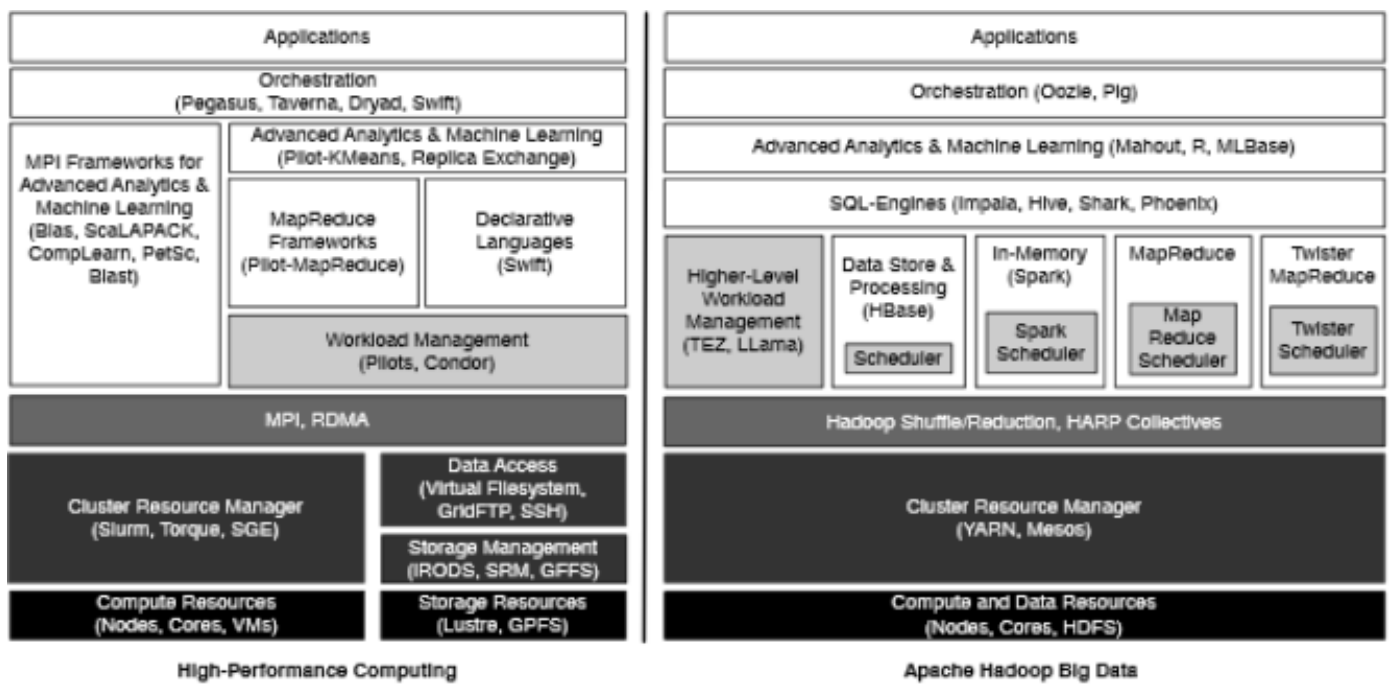

Figure 2.1: Typical HPC and BDA software stacks.

get value from HPC, it is often not enough to run a model. One has to collect the data, transform it into a form the model can accept, run the model, interpret the results, and finally present them in a form that people can use.

In this chapter we compare the typical HPC and BDA stacks, and identify storage as a strong candidate for HPC and BDA convergence. Indeed, the challenges faced on both sides and directions taken are strikingly similar: trading versatility for performance. As such, we advocate that the storage layer offers strong opportunities for convergence.

\subsection{Comparative overview of HPC and BDA stacks}

Significant differences exist between HPC and BDA platforms. While HPC mostly focuses on large computational loads, BDA targets applications that need to handle very large and complex data sets; these data sets are typically of the order of multiple petabytes or exabytes in size. BDA applications are thus very demanding in terms of storage, to accommodate such a massive amount of data, while HPC is usually thought more in terms of sheer computational needs. On Figure 2.1 we highlight the stacks that are typical for HPC and BDA applications.

These key differences between both paradigms result in very different sets of key requirements. A traditional cloud-based BDA platform offers features that are attractive to the general public. These services comprise single, loosely coupled instances (an 
instance of an OS running in a virtual environment) and storage systems backed by service level agreements (SLAs) that provide the end user guaranteed levels of service. These clouds are generally designed to offer the following features:

Instant availability: Cloud offers almost instant availability of resources.

Large capacity: Users can instantly scale the number of applications within the cloud. Software choice: Users can design instances to suit their needs from the OS up. Virtualized: Instances can be easily moved to and from similar clouds.

Service-level performance: Users are guaranteed a minimal level of performance.

Although these features serve much of the market, HPC users generally have a different set of requirements:

Close to the hardware: HPC libraries and applications are often designed to work closely with the hardware, requiring specific OS drivers and hardware support.

Tuned hardware: HPC hardware is often selected on the basis of communication, memory, and processor speed for a given application set.

Tuned storage: Storage is often designed for a specific application set and user base. Userspace communication: HPC user applications often need to bypass the OS kernel and communicate directly with remote user processes.

Batch scheduling: All HPC systems use a batch scheduler to share limited resources.

These different sets of requirements have a significant impact on the data storage layer for HPC and BDA platforms, which is largely divergent at the same time in terms of architecture and design principles. Many reasons explain storage divergence between HPC and BDA. This includes the different software development models, virtualization, scheduling, resource allocation, stateful networks vs. stateful services. Another key difference is the specifics of the data, which has a significant impact in the underlying software and hardware infrastructure, or stack. A stack can be seen as a set of different components, including operating system, execution framework, provisioning, remote console or power management, cluster monitoring, parallel file system and scheduling, development and performance monitoring tools enabling the final users to interact with the cluster, as well as the underlying hardware parts such as CPU, memory and networking. 
In the following sections we zoom on the key differences between HPC and BDA storage stacks.

\subsection{HPC: Centralized, file-based storage}

HPC applications tend to be used for computational analysis, data-intensive research, rich media, three-dimensional computer modelling, seismic processing, data mining and large-scale simulation. Driven by CPU-intensive processing, such applications handle large volumes of data over short periods of time while also in some cases permitting simultaneous access from multiple servers. The need to process large volumes of data quickly has huge repercussions for HPC storage, given that storage I/O capabilities are typically much lower than those of processors. An HPC storage system needs large capacity accessible at high speed and to be highly expandable, while offering a single global namespace accessible to all users involved in the project.

State-of-the-art storage systems for high-performance computing (HPC) are mainly comprised of the parallel file systems deployed on remote storage servers. That is, the compute and storage resources are segregated, and interconnected through a shared high-performance network. This architecture is explained by two main factors. First, many legacy scientific applications are compute-intensive, and barely touch the persistent storage except for initial input, occasional checkpointing, and final output. Therefore the shared network between compute and storage nodes does not become a performance bottleneck or single point of failure. Second, a parallel file system proves to be

highly effective for the concurrent I/O workload commonly seen in scientific computing. In essence, a parallel file system splits a (large) file into smaller subsets so that multiple clients can access the file in parallel.

While large-scale applications are becoming more data-intensive, moving very large amounts of data in and out of the remote file system is becoming a significant performance bottleneck, greatly hindering time to results. Hence researchers spend significant efforts on improving the I/O throughput of the aforementioned architecture by means of various techniques, such as burst buffers (105). 


\subsection{BDA: Modular, application-purpose storage}

On the other hand, BDA analytics architectures have been built from the ground up to be able to grind through enormous amounts of data with the highest possible performance. The data motion issue that arises in HPC systems is solved by bringing the computation to the storage nodes rather than the opposite. This principle has driven the design of the underlying storage stack.

While multiple BDA frameworks exist, Hadoop (145) is arguably the one that impacted most the architecture of BDA platforms today. Hadoop is a software solution that was developed to solve the challenge of doing a rapid analysis of vast, often disparate data sets. The results of these analytics, especially when produced quickly, can significantly improve an organization's ability to extract knowledge from the aforementioned data.

Hadoop embraces bringing the computation to the data. Essentially, Hadoop was originally designed to leverage multiple compute nodes. Each of these nodes has its direct attached storage (DAS) which has to be large enough to store the data of any assigned analytics job. One of the nodes in a Hadoop cluster, called the master node, is in charge of coordinating all these nodes. It also maintains a large amount of metadata so that it understands which data is where on the cluster and what node is the most available. The master node assigns the analysis of an analytics request to a node within its cluster that has the appropriate data on its internal storage. When the job is complete, the result is sent back to the master node and given to the user. The concept behind this architecture is it can be assembled inexpensively, with standard off-the-shelf hard disk and flash drives and no expensive networking requirements.

Hadoop abstracts the storage layer access by proposing an extendable set of data access strategies that essentially separate the computation from the storage. This brings various benefits such as transparent application portability across platforms and independence of the underlying storage system.

File-based storage are arguably the de facto standard in the industry. The Hadoop Distributed File System (HDFS) (145), for example, is an integral part of the Hadoop stack. However, most data warehousing solutions today tend to also offer connectors enabling users to analyze vast amounts of data originating from a wide range of storage systems and paradigms. These systems include key-value and columnar 
stores (Riak (86), Cassandra (92), Aerospike (148)), object stores (Amazon S3 (20), Ceph (167)) or time series databases (OpenTSDB (16)).

\subsection{Conclusions: challenges of storage convergence be- tween HPC and BDA}

While designing a common storage solution for HPC and BDA could improve portability of application between platforms, the challenges of fitting the requirements of both communities are hard (134). Indeed, the broad variety of storage systems available for HPC and BDA exhibit different characteristics or deployment models targeted at a specific range of applications. Defining a storage solution able to combine the requirements of both HPC and BDA applications is challenging. For example, it is unclear which storage paradigm is a natural fit for handling data in a broad range of scientific application codes, nor the extent of the code modification required to do so. In particular, there is a tension between the colocation of data and computation that is embraced by cloud platforms and the contradictory dynamic task scheduling and execution that is the core to HPC storage architectures (63). Also, while file-based storage is common between HPC and BDA stacks, many differences exist in their design principles and implementation. Additionally, BDA embraces a wider variety of storage options such as key-value stores or object stores.

Consequently, in order to achieve storage-based convergence between HPC and $\mathrm{BDA}$, it is critical to evaluate which storage paradigm is better suited to answering the precise requirements of both communities and applications. 


\section{Chapter 3}

\section{Distributed storage paradigms}

Distributed storage services are generally classified by the way they are consumed and interfaced with on the client side. Although file storage and key-value systems have been used for decades, the explosion of unstructured data, the proliferation of globally distributed cloud based architectures, and the need for more simplistic and low cost storage solutions gave momentum to a new type of storage called object storage.

Common to all these paradigms are two key notions for most persistent storage systems: fault-tolerance and scalability. Let us define these notions:

Fault-tolerance: A fault tolerant system should be immune to transient or partial failures. These faults include: network and server failures causing data or service unavailability, data consistency and integrity under concurrent user access.

Scalability: A scalable system is able to harness extra resources added to increase network storage capacity, increasing performance and addressing the need for supplementary storage. When these additional resources are provided by upgrading existing hardware, the system is deemed to be vertically scalable. In contrast, a horizontally-scalable system, also referred to as a scale-out system, these new resources are provided by connecting additional machines to the resource pool.

In this chapter we discuss the differences between file storage, key-value stores, and object storage in order to understand the benefits and limitations of these approaches in the light of HPC and BDA convergence. 


\subsection{Distributed file systems}

A distributed file system organizes data as files in folders as a hierarchy of directories, subdirectories and files that use naming conventions based on characteristics such as extensions, categories or applications. It provides a permanent storage in which a set of objects that exist from their explicit creation until their explicit destruction are immune to systems failures. This permanent storage consists of several federated storage resources in which clients can create, delete, read or write files. Unlike local file systems, storage resources and clients are dispersed in a network. Files are shared between users in a hierarchical and unified view: files are stored on different storage resources, but appear to users as they are put on a single location.

The distributed nature of distributed file systems usually remains transparent to the user, hiding the complexity of the underlying system. They should access the system regardless where they log in from, be able to perform the same operations on distributed and local file systems, and should not care about faults due to distributed nature of the file system thanks to fault-tolerance mechanisms. Transparency can also be seen in terms of performance: operations on a distributed file systems are expected to show at least similar performance as the same operations executed on a local file system.

\subsubsection{A common standard: POSIX-IO}

The transparency of distributed file systems is largely due to them implementing part of all of the POSIX I/O standard, which has defined the expected API and behavior of local systems for decades. At a high level, POSIX I/O is composed of two distinct components that are often conflated: POSIX I/O API and POSIX I/O semantics.

The POSIX I/O API essentially defines how applications read and write data. It encompasses many of the calls any scientific programmer should be familiar with, including open, close, read, write, and 1seek, and as such, are an integral part of today's applications and libraries. The API defines key features provided by the file system, such as hierarchical namespace or permissions.

In contrast, the POSIX I/O semantics define what is and is not guaranteed to happen when a POSIX I/O API call is made. For example, the guarantee that data has been committed to somewhere durable when a write call returns without an error 
is a semantic aspect of the POSIX write API. While this notion of write being honest about whether it actually wrote data may seem trivial, these semantics can incur a significant performance penalty at scale despite not being strictly necessary for many applications.

While most distributed file systems strictly adhere to the POSIX I/O API, the compliance to the associated semantics greatly varies from system to system. Key differences between various systems are in their consistency semantics and in their caching and locking policies. For example, PVFS (137) does not support POSIX I/O atomicity semantics. Instead, it guarantees sequential consistency only when concurrent clients access non-overlapping regions of a file (referred to as non-conflicting writes semantics). On the other hand, Lustre (142) guarantees consistency in the presence of concurrent conflicting requests.

It is worth to note that some file systems choose to offer only a subset of the POSIX I/O API. This is most notably in the case of the Hadoop Distributed File System (HDFS) (145). Indeed, the Hadoop data analytics framework is purpose-built for Hadoop workloads. Hence, it does not need random file update semantics that are core to POSIX I/O. This significantly eases the design of the file system by eliminating the synchronization required for handling potential conflicting write operations.

\subsubsection{Various data and metadata management architectures}

Although most of the existing file systems adhere to part or all of the POSIX I/O standard, the underlying architecture permitting them to do so greatly varies. This architecture also significantly impacts the performance and the level of POSIX I/O compliance of the system. Five main distributed file system categories can be identified (158):

Client-Server File Systems: A client-server file system exports a standardized view of its local file system to remote clients. The associated communication protocol enables a variety of heterogeneous clients to share the same namespace to store and retrieve information. Because of its simplicity, it remains widely used today despite its weaknesses. Those include solely relying on local mechanisms such as RAID (129) to provide fault-tolerance, offering constrained performance under high load or only permitting vertical scalability because of the single-server nature 
of these file systems. Common examples of client-server file systems are NFS (139) or CIFS (98).

Cluster File Systems: In a cluster file system, the namespace is the aggregation of resources from multiple federated servers connected over the network. This resource aggregation is kept hidden from the user. Compared to client-server file systems, the multiple-server nature enables relying on additional mechanisms to provide a higher level fault-tolerance, such as data replication. A key feature offered by most cluster file systems is their horizontal scalability achieved by connecting additional servers to the federation. Most distributed systems in use today are cluster file systems. Examples include PVFS (137), Lustre (142), GPFS (140), GlusterFS (68) or Ceph (167).

Asymmetric File Systems: An asymmetric file system is a file system in which there are one or more dedicated metadata managers that maintain the file system and its associated disk structures. This architecture greatly eases providing POSIX I/O semantics, but may present and additional bottlenecks and scalability challenges because of the need to separately scale metadata and data management systems. This is the case of the vast majority of distributed file systems, including HDFS (145), Lustre (142) or GPFS (140).

Symmetric File Systems: A symmetric file system distributes data and metadata over all servers nodes of the system in such a way that all nodes are able to understand the disk structure. This metadata distribution and the associated look-ups are implemented using peer-to-peer (P2P) protocols. Such architecture enables horizontal scalability, but substantially complexifies the underlying design of the system. Notable examples of symmetric file systems include PVFS (137) or GlusterFS (68).

Parallel File Systems: In a parallel file system, the data blocks are striped, in parallel, across multiple storage devices on multiple storage servers. This architecture enables distributing the concurrent load from multiple clients across multiple servers, consequently resulting in improved multi-user capabilities. In addition, this parallelism permits storing files whose size exceeds the storage capabilities of a single server. However, parallel file systems raise additional challenges to 
guarantee that the stripes are synchronized and consistent. The vast majority of cluster file systems in use today are parallel (136).

Each distributed file system can be defined as a combination of these generic types. Each showing different characteristics with respect to performance, scalability or faulttolerance, the choice of the appropriate file system for a specific target use-case is key to designing large-scale data storage platforms.

\subsubsection{Limitations}

While file systems exhibit strong benefits for managing and organizing data, these benefits are usually shaded by significant performance and scalability limitations as the data size grows. These limits are the result of their design.

First, POSIX I/O is almost universally agreed to be one of the most significant limitations standing in the way of I/O performance for large exascale systems. This is due to various constraints it poses relative to the behavior of the file system when shared by multiple concurrent users. This includes its stateful nature, prescriptive metadata or its often-unneeded strong consistency when facing with concurrent, conflicting writes. While these features are not problematic by themselves, they imply a substantial performance cost that the users have to pay no matter their actual requirements. For that reason, several file system choose to relax several parts of the POSIX I/O standard to offer increased performance for specific use-cases where these features limit performance for no tangible benefit (137). Also, various extentions to the POSIX I/O standard have been proposed in the literature to achieve similar results (19; 90; 163).

Yet, the most significant limitation with respect to performance can be seen as the hierarchical nature of file systems itself, which is at the very root of their design. Indeed, when accessing any piece of data, its associated metadata must also be fetched. In a hierarchical design, this implies fetching multiple layers of metadata. In asymmetric file systems, metadata operations tend to become a bottleneck at scale because of the concentration of requests to a small number of machines. In symmetric file systems, this bottleneck effect is reduced at the cost of a higher access latency due to the distribution of the metadata across the whole system, which only increases with the size of the system and the hierarchical depth of the files that are accessed. 
These two factors inherently limit the scalability potential of distributed file systems, either making them unsustainable for exascale applications or requiring significant changes to the very design of these systems. This ultimately negates the very reason they were initially built for: providing a simple and efficient way for users to manage shared data.

\subsection{Key-value stores}

Key-value stores (or key-value databases) propose a fundamentally simpler data representation model compared to distributed file systems. They emerged as an efficient replacement for relational databases which often fail to meet the velocity requirements of BDA for simple use-cases where relationality is not critical. Key-value stores are the simplest of the NoSQL (Not Only SQL) database paradigms (99).

At a high level, key-value stores propose an associative array as the fundamental data model, in which each key is associated with one and only one value in a collection. This relationship is referred to as a key-value pair. In each key-value pair the key is represented by an arbitrary string. The value can be any kind of data like an image, user preference file or document. The value is opaque to the key-value database requiring no upfront data modeling or schema definition. Some popular key-value stores are Dynamo (55), Cassandra (92), Riak (86) or Aerospike (148).

Two fundamental differences exist with respect to distributed file systems. First, the key-value pairs are organized in a flat namespace, hence avoiding the performance penalty of managing large, deep hierarchies altogether. Second, while the basic set of operations is roughly similar across systems, no fixed standard exists that would constrain the exact set of features and semantics offered by each system. Consequently, a wide variety of key-value stores exists, each exhibiting potentially a different set of features and semantics suited to different ranges of applications.

\subsubsection{Minimal API and semantics}

As a fundamental data representation or data structure, the API and semantics offered by key-value stores are generally very simple. They provide a way to store, retrieve and update data using simple commands; the path to retrieve data is a direct request to the object in memory or on disk. The minimal API usually consists of these operations: 
Add: Add a key-value pair to the collection of data,

Reassign: Change or reassign the value associated with a key; in practice this operation is often the same as add,

Remove: Remove a key-value pair from the collection of data,

Get: Get the value associated with a key.

This API is often associated with simple semantics: all operations are atomic, stateless and idempotent. Consequently, the challenges associated with sharing a single key-value store with multiple users and coordinating concurrent requests is minimal. The simplicity of this model also makes a key-value store fast, easy to use and flexible. Yet, the variety of available systems with no defined standard may intuitively come at the expense of portability. However, in practice, the inherent simplicity of the data model makes the portability of an application to a different key-value store simple, or even transparent with an appropriate thin abstraction layer.

\subsubsection{Scalability}

A key-value store distributes the key-value pairs across the whole system, enabling them to be retrieved using $\mathrm{P} 2 \mathrm{P}$ protocols. This data distribution is very similar to the metadata storage architecture of symmetric file systems. Associated with their flat namespace, this enables key-value stores to exhibit nearly infinite horizontal scalability (76).

Another important aspect of the simplicity of the API and semantics offered is the ability to shift part of the work associated to data operations to the user. Indeed, a client usually communicates with all server instances instead of a single entry point, and is given sufficient knowledge about the data distribution across the cluster to pinpoint the server hosting any piece of data it wants to read or delete independently of any dedicated metadata server. This enables key-value stores to reduce the cost and the latency associated with most storage operations, and further improves their horizontal scalability.

\subsubsection{Limitations}

The main limitation associated with key-value stores is the result of this very simple $A P I$. Specifically, modifying a key-value pair is only possible by replacing the key as a 
whole. In practice, this limits key value stores to two usage scenarios: small data pairs or static content. Most key-value store are indeed designed to handle relatively small data objects, with a typical size ranging from a few bytes to a few megabytes.

Some key-value stores are specifically designed to handle large data objects by leveraging data striping, such as Amazon S3 (20). Given the lack of update operation, they often represent static content such as pictures or videos. Such key-value stores are usually referred to as object stores.

This makes key-value stores unfit for a wide range of use-cases. This is a significant issue for HPC applications that frequently need to update potentially large output files generated, for example, by large-scale simulations or event generators.

\subsection{Document, columnar, graph databases}

A wide variety of storage models exist between the complex schemas of relational databases and the extreme simplicity of key-value stores. This is the case for applications that require structure in the stored data. Among the most popular of these models are document databases, wide column databases or graph databases. The main design objective of these stores is to provide a "best of both worlds" approach between relational databases and key-value stores. Indeed, a common characteristic shared by these data models is the shift from an opaque value to a structured value understandable by the storage system. The storage system is hence able to use this structure to extract metadata, perform optimization or provide the user with advanced query functionality.

\subsubsection{Document databases}

Document databases extend key-value stores by enabling users to store documents instead of values. A document is a data object whose structure can be understood by the storage system, usually JSON (33) or XML (34). The structure of these documents often permits richer semantics than relational databases, offering for instance nested documents or data structures such as associative arrays or lists. This rich object definition enables complex documents to be represented as a single object instead of being spread across multiple tables, hence eliminating relational mapping. Documents do not 
have to adhere to a pre-defined schema, nor are they expected to contain the same set of keys, or structure.

Document databases are able to offer more features than key-value stores by leveraging the structure of the data, contributing to a richer experience. Such features include the possibility to query or update only parts of a document, to filter results or to execute complex queries using database-managed secondary indexes. In practice, the set of query API or query language features available, as well as the expected performance of the queries, vary significantly from one system to another. Likewise, the specific set of indexing options and configurations that are available vary greatly by implementation.

Among the most popular document databases are MongoDB (45), CouchDB (21) or RethinkDB (161). Document databases are also available as cloud software-as-a-service (SaaS) offerings with Amazon SimpleDB (133) or Azure Cosmos DB (130).

\subsubsection{Wide column databases}

Wide column databases take document databases one step closer to relational databases, or specifically to column-oriented relational databases. The data model offered by this type of storage system is very similar to that of relational databases, being composed of a set of tables containing columns. Wide column stores embrace a hybrid approach between document and relational databases mixing the key-value pair based and variables schema of the former with the declarative characteristics of the latter. Wide column databases adopt a storage model similar to column-oriented relational databases. Indeed, wide column databases store data tables as sections of columns of data rather than as rows of data. This data layout yields performance advantages for large-scale data analytics, where only a subset of the columns has to be scanned.

Because of its performance for large-scale analytics, this type of data stores is extremely popular in the context of BDA (38). The first wide column database, Big Table (44) was introduced by Google to complement its proprietary Map-Reduce implementation. Yahoo! later introduced the Hbase (65) data store for the Hadoop framework, while Facebook developed Cassandra (92) to serve their own data analytics needs. These databases retain the high scalability of key-value stores. For example, Cassandra has been deployed by Apple on clusters composed of up to 75,000 nodes and hosting more than $10 \mathrm{~PB}$ of data (159). 
Other databases of this type include Apache Accumulo (143) or ScyllaDB (109), as well as SaaS offerings, such as Azure Tables (66).

\subsubsection{Graph databases}

Compared to other types of storage systems, graph databases make the relations (or edges) between individual documents a first-class citizen. Such relations allow to assemble individual documents (or nodes) as a complex graph. This obviates the need for manually inferring relations between documents, using special properties such as foreign keys, joins or out-of-band processing such as Map-Reduce. Graph databases are often schema-less - allowing for the flexibility of a document database - but supporting relationships in a similar way to that of a traditional relational database. Graph databases offer a specific query language dedicated to traversing the data structure. Hence they excel for a range of applications such as social networking, pattern discovery or deep data traversal.

This query power however comes at the cost of scalability. Indeed, graph databases are notoriously hard to scale horizontally for the same reason relational databases are. To ensure the best performance, the graph should be distributed for the frequently co-accessed data to be co-located. Yet, partitioning a data set composed of connected entities is a NP-hard problem (84). The partitioning method impacts the final query performance, and needs to be fine-tuned for the expected workload (170). For that reason, Neo4j (165) or Amazon Neptune (7) do not partition the graph, hence targeting vertical scalability. Distributed graph databases such as Titan (121) offer horizontal scalability at the cost of performance for deep traversals.

\subsubsection{Limitations}

The main limitation of document, wide column and graph databases lies in the very reason they are popular for web application development: the data structure they expect makes them unfit for handling large, unstructured data. These databases trade versatility for expressiveness to various extents, which in practice limits their scope of application. Yet, interestingly, these NoSQL paradigms can be implemented as thin layers over a simpler, opaque storage layer. This is for example the case of the multimodel OrientDB storage system (155), offering both a document database and a graph database over a common storage layer, or HBase, which offers a wide column store over 
HDFS. The Titan graph database is in turn implemented as a layer above a pluggable backend such as HBase or Cassandra.

Furthermore, similarly to key-value stores, these storage models are typically targeted at storing relatively small data objects, with a size ranging from a couple of bytes to a few megabytes. This is well below the data object size that is typical for both HPC and BDA storage systems (138).

\subsection{Blob storage}

A key observation that can be made from the limitations of distributed file systems and key-value stores is that they are relative to different aspects of the systems. Indeed, distributed file systems are mostly limited by their hierarchical nature and semantics, that is, the way they manage metadata. In contrast, key-value stores limitations derive from the insufficient set of operations permitted on the data. As such, it is possible to design a storage system combining most of the advantages of both distributed file systems and key-value stores. Such storage systems are named blob stores (Binary Large Objects), or object stores. They combine the flat namespace and small API that make key-value stores efficient and highly-scalable with the update operations that make distributed file systems adaptable to a wide range of use-cases. They are usually referred to as blob storage systems.

Typical examples of such blob storage systems include RADOS (167), or hosted services available on cloud platforms such as Microsoft Azure Storage Blobs (39) or Amazon S3 (20).

\subsubsection{Data model}

The data model and the API of blob storage systems are largely similar to that of keyvalue stores. The two major changes relate to the way a piece of data can be read from or written to. Indeed, a blob storage system offers the possibility to overwrite only a part of the data associated with a key. Symmetrically, the read operation is updated to allow retrieving only a part of the data. Associated with the data striping found in parallel file systems, this API change effectively enables blob storage systems to be used with large, mutable data objects. Blob storage systems retain the two features of keyvalue stores explaining their scalability: flat namespace and decentralized architecture. 


\subsubsection{Semantics and concurrency control}

State-of-the-art blob storage systems such as RADOS generally retain the consistency guarantees of key-value stores, enabling writes on an object to be performed atomically. There exists one significant exception to this rule: striping large data objects across multiple servers raises additional challenges when a write operation spans multiple data chunks. Indeed, as two data chunks are functionally separate objects potentially located on different servers, strict consistency control is necessary to guarantee the correctness of this operation when processing concurrent, conflicting requests. This consistency management generally comes at the cost of performance. In practice, most object storage systems make no guarantee regarding the correctness of operations spanning multiple chunks (13), requiring application developers to take special care when using such storage systems in a multi-user, shared context.

Some blob storage systems strive to offer higher consistency guarantees by embracing a version-based metadata management, enabling multiple versions of a blob to co-exist on the system at any given time. This is notably the case of BlobSeer (127), which embraces Multi-Version Concurrency Control (MVCC) (26) to cope with concurrent, conflicting requests. BlobSeer distributes the version tree across the whole cluster in order to achieve high horizontal-scalability.

\subsubsection{Modular storage}

The simple yet versatile data model and API offered by blob storage systems enables them to be used already in a variety of applicative contexts. Most notably, they can serve as building blocks for different storage abstractions, enabling developers to focus on high-level design rather than on low-level storage.

Additionally, blob storage systems are increasingly used to serve as the underlying data storage layer for distributed file systems. For example, Ceph (167) relies on the purpose-designed RADOS distributed blob storage system to manage the data distribution and persistence across the cluster. Although not distributed, the Object Storage Devices (OSDs) components of Lustre (142) serve a similar purpose. RADOS offers additional storage abstractions such as the Ceph Object Gateway (object storage) or Ceph Block Device. 


\subsubsection{Limitations}

Blob storage systems combine positive aspects of both distributed file systems and keyvalue stores, but to some extent, they also combine their drawbacks. Most notably, performance-wise, the features that are added on top of key-value stores invariably impact the performance of the system. Indeed, some form of concurrency control is necessary to provide ordering between concurrent requests. In BlobSeer, the distributed metadata management used to guarantee the correctness of operation spanning multiple chunks is inherently incompatible with the client-side data location. This significantly increases the overall system latency because of the necessary metadata fetch for each operation. This metadata access cost is in practice comparable to that experienced with symmetric distributed file systems. When such correctness guarantees are not provided as in the case of RADOS, this requires application developers to take special care regarding request coordination to prevent data to be corrupted by concurrent, conflicting write operations.

Overall, flat namespaces are friendly to applications by offering low data-management overhead. Yet, this comes at the expense of user experience by not providing efficient ways for humans to manage the data using an intuitive interface such as a simple hierarchical file explorer for file systems. This may quickly become an issue when the number of objects in a namespace grows, calling for some external metadata management system to be implemented.

When a storage system is deployed in a multi-user context, for instance on a supercomputer, access control and security need to be addressed. The lack of folder hierarchy implies access control to be implemented at the object or at the namespace level. Considering that separately managing permissions for potentially large amounts of objects is challenging by itself, namespace-level permissions will generally be considered. This effectively requires blob storage systems to handle multiple namespaces concurrently. While not a technical challenge by itself, to the best of our knowledge, none of the state-of-the-art storage systems available do implement this feature. 


\subsection{Conclusions: which storage paradigm for converging storage?}

Most HPC and BDA platforms today still rely on distributed file systems, which used to provide a good balance between performance and versatility. However, the fast growth of the data volumes and throughput requirements of data-instensive applications running on a new generation of extreme scale platforms pushes this storage paradigm to its limits. As the research investments necessary for these systems to cope with such challenging requirements grow, there is a clear trend in both HPC and BDA communities to move away from distributed file systems altogether.

While key-value or other NoSQL data stores provide a highly efficient data model able to cope with more than a billion I/O operations per second on a single server (104), they do lack support for the large, mutable data objects required by a wide range of extreme scale HPC simulations and industry standard I/O frameworks.

Such support for large objects is precisely the main target of blob storage systems. In this context, they would provide a middle-ground approach combining the data model required for HPC applications, exhibiting a horizontal scalability on par with that of keyvalue stores and a virtually infinite capacity critical for BDA applications. Furthermore, the deployment of blob storage does not conflict with legacy applications due to their capability to serve as a building block for highly efficient file system interfaces. As such, blob storage systems constitute a strong candidate for achieving storage-based convergence between HPC and BDA.

However, despite being a very promising alternative to distributed file systems, blob storage systems are not exempt for limitations. Specifically, we have seen that significant challenges lie in their consistency guarantees when faced with concurrent, conflicting writes. While some systems choose not to provide any such guarantees, some do so at the expense of a substantial storage operation latency increase compared to key-value stores. For blob storage systems to be proposed as an alternative to file systems for both HPC and BDA, we believe that a middle-ground approach is here also required: providing configurable consistency guarantees while only exhibiting minimal overhead when no such guarantees are required. 


\section{Chapter 4}

\section{Consistency management}

One of the storage guarantees that is required by virtually any application is data consistency. Despite being a common requirement for storage systems, providing this guarantee has always posed hard challenges to system developers because of the wide range of conditions that can potentially result in data corruption. Besides system and network failures common to all systems, concurrent, conflicting storage requests can also yield incorrect results. This is the case when the storage system purposely relaxes these guarantees, such as Ceph (167) for writes operations crossing a chunk boundary (13). Data corruption may also occur when a series of storage requests is incorrectly expected to be executed atomically by the system, as with concurrent readupdate-write requests (23). The latter case requires the user to prevent two conflicting requests from ever executing simultaneously. This can be ensured either by relying on applicative locks or by leveraging synchronization primitives provided by a layer down the storage stack. Defining at which layer to implement consistency management is a hard question. In this chapter we discuss the solutions generally adopted by HPC and BDA developers, which significantly diverge.

As in any storage stack, ensuring data consistency in a convergent HPC and BDA storage system is critical. Integrating consistency management however is not an easy task. Indeed, HPC and BDA rely on opposite principles with respect to data consistency. The former will typically leverage a storage system offering POSIX I/O strong consistency while leave up to the user to use high-level synchronization tools defined in the framework or directly inside the application when required. The latter usually relies on a wide variety of storage systems offering low-level consistency depending on 
the application requirements. The systems offering strong consistency often take care of request synchronization based on user-provided semantics when required.

In this chapter we provide an overview of the mechanisms used on each side to ensure data consistency to understand their advantages and inherent limitation in the light of HPC and BDA convergence.

\subsection{Storage consistency models}

Central to the consistency guarantees made by any storage system is the consistency model it provides. A consistency model is a contract between a distributed data store and a set of processes, which specifies what the results of read/write operations are in the presence of concurrency (124). It defines the general guarantees made by the storage system regarding the consistency a client can expect when dealing with concurrent, conflicting requests. Such conflicting requests can be either read-write conflicts (concurrent read and write operations) or write-write conflicts (two concurrent write operations) on the same data object.

Consistency models are split in two general categories: strong consistency and weak consistency. The former guarantees that all accesses are seen by all clients processes in the same order (sequentially). The latter relaxes this guarantee by allowing different clients to perceive the same variables in different states (58).

\subsubsection{Strong consistency models}

Ideally, a storage system can hide the fact that it is replicated from clients and act indistinguishably from a storage system running on a single computer. The replication would increase the system's fault-tolerance but would not cause it to expose any abnormal behavior to clients. Strong consistency allows different behavior regarding the ordering of operations:

Strict consistency: A read operation must return the result of the latest write operation which occurred on the data item. This is the strongest possible consistency model. It is only possible with a global, perfectly synchronized clock, with all writes instantaneously visible to all clients. It is therefore not applicable to distributed systems; it is the consistency model of uniprocessor systems. 
Linearizability: Writes should appear to be instantaneous. Imprecisely, once a write completes, all later reads (where "later" is defined by wall-clock start time) should return the value of that write or the value of a later write. Once a read returns a particular value, all later reads should return that value or the value of a later write (79). It is the consistency models of transactional systems such as Spanner (50) or HyperDex (60).

Sequential consistency: The result of any execution is the same as if the operations of all the processors were executed in some sequential order, and the operations of each individual processor appear in this sequence in the order specified by its program (93). Compared to linearizability, sequential consistency does not make guarantees regarding the relative ordering of requests originating from different clients. This consistency model is that of the ZooKeeper distributed key-value store (80). It is also the consistency model imposed by the POSIX I/O standard (88) and therefore that of Lustre (142), GPFS (140) or PVFS (137).

\subsubsection{Weak consistency models}

The weak consistency models differ in the precise details of which operation reorderings are allowed within the activity of a client, and in whether there are any constraints at all on the information provided to different clients. These relaxed consistency models enable in some cases to overcome the performance penalty incurred by the strong consistency guarantees.

Eventual consistency: If no updates take place for a long time, all replicas holding the same data will gradually converge to the same value. It is the consistency model of epidemic protocols, and is very common in NoSQL stores such as Dynamo (55), Cassandra (92) or Riak (86).

Causal consistency: All writes that are (potentially) causally related must be seen by every process in causal order (81). This is for example the case with a read followed by a write on the same value from the same client, or with a write followed by a read of the same value from different clients. Causal consistency is the foundation of the COPS key-value store (106). 


\subsection{Application-specific consistency management}

Any application requiring higher storage consistency guarantees than those provided by the underlying storage system needs to incorporate the mechanisms targeted at achieving this level of consistency. For example, read-update-write operations require the atomic execution of the read and write operations as a single unit. Should the storage system not provide the tools to express such consistency requirement, the application developer needs to ensure at the application level that this atomic execution is enforced. To the best of our knowledge, no POSIX I/O-compliant distributed file system, weakly-consistent object or blob storage do provide such semantics today. Two main techniques are used to ensure such atomicity: either locking, or avoiding concurrent requests altogether.

\subsubsection{Application-Level Locking}

Application-level locking is the most intuitive way of ensuring that multiple storage requests are executed atomically. Essentially, locks create a protected region in the application code that cannot be executed by more than one process at the same time. When accessing this protected region, a process atomically acquires a lock on this region. Any concurrent process trying to access the region while it is locked is paused until the region is unlocked by the process holding the lock.

Application-level locking is an intuitive way to express consistency requirements, allowing the developer to fine-tune the locking mechanisms according to his knowledge of the application internals and requirements. Yet, besides its negative performance impact, application-level locking is not exempt from weaknesses. First, for non-trivial applications, designing lock-based consistency management is difficult and error-prone. Indeed, one needs to ensure the highest-possible lock granularity to not hinder application performance beyond the necessary, and at the same time handling all potential failure conditions leading to deadlocks.

Furthermore, locks do only provide atomicity from the point of view of the application; the storage system is oblivious to the semantics expressed by application-level locks. Hence, the data can become corrupted by only partially-executed protected regions during failure scenarios of the storage system, of the application or of the network. 


\subsubsection{Deferred consistency}

In some cases, an application only needs to ensure consistency of the storage at a specific point in time. This is for example the case when multiple ranks of an HPC application are able to work independently from each other, only merging the data after the data processing finishes. This is usually referred to as deferred consistency.

This form of consistency intuitively yields optimal I/O performance. Yet, in many cases, it requires designing the application in a way that is not intuitive or natural to the application developer. Not all applications can be expressed in such a way, limiting its application to a few specific use-cases.

\subsubsection{I/O proxying}

When an application needs to collaborate on writing to a single object in a way that could result in data inconsistency, one can use one or multiple processes to perform $\mathrm{I} / \mathrm{O}$ operations on behalf of the other processes. Each process is assigned an exclusive region of the data objects, so that only one node can write to any given region at the same time. Such technique is often implemented in data management frameworks such as in MPI I/O collective operations (157).

The main drawback of this technique is its substantial performance cost resulting from the additional network requests required to perform I/O operations, or if a single I/O node becomes overloaded. Special care needs to be taken when implementing I/O proxying in order to ensure the fault-tolerance of the application.

\subsubsection{Limitations}

Overall, application-level locking offer the highest potential for optimization by the application developer. Indeed, all three techniques can be fine-tuned to the exact requirements of the application. In most cases however, this will require a substantial development and debugging effort that is only valuable for very large, highly throughputsensitive applications. In particular, such investment is rarely adapted to fast-evolving BDA applications, and in which scalability is favored over bare performance. 


\subsection{Transactional semantics}

In a transactional model, the role of enforcing data consistency rules is left to the storage system. The user specifies these rules using a simple API that informs the storage system of the expected semantics for any given set of request. Essentially, a transaction is a set of storage operations that are to be processed and executed as an atomic unit, as a single logical operation of the data.

Two major sets of properties exist to define the execution guarantees for any storage operation provided by transactional systems: ACID and BASE.

\subsubsection{Strong consistency with the ACID model}

The strongest of the two models is known as ACID (75). It states that the four following properties must be satisfied for every operation:

Atomicity: All of the operations in the transaction will complete, or none will. If one part of the transaction fails, the entire transaction fails.

Internal Consistency: The storage will be in a consistent state when the transaction begins and ends. This property ensures that any transaction will bring the storage from one valid state to another. This rule must be satisfied for all nodes in a cluster. Internal consistency relates to the preservation of invariant relations that exist among items within a database, and is consequently largely dependent on the application semantics (160).

Isolation: The transaction will behave as if it is the only operation being performed on the data. Each transaction has to execute in total isolation from concurrently running operations.

Durability: Upon completion of the transaction, the operation will not be reversed.

ACID has been the cornerstone of relational databases for decades. It is increasingly being used in a variety of non-relational systems such as key-value stores (153; 61; 141) or even file systems (59). 


\subsubsection{Relaxing consistency with the BASE model}

A key observation leading to the BASE model (131) is that not every application truly needs ACID-compliant transactions. Relaxing on certain ACID properties has opened up a wealth of possibilities, which have enabled some data stores to achieve massive scalability and performance for their applications. Whereas ACID defines the key characteristics required for reliable transaction processing, they inherently require the storage system to satisfy the offer strong consistency and hence to sacrify availability. These opposing characteristics are captured in the acronym BASE:

Basically Available: The system is guaranteed to be available for querying by all users. (No isolation.)

Soft State: The values stored in the system may change because over time per the eventual consistency model described below.

Eventually Consistent: As data is added to the system, the system's state is gradually replicated across all nodes. For example, when a file is written to HDFS, the replicas of the data blocks are created in different data nodes after the original data blocks have been written. For the short period before the blocks are replicated, the state of the file system is not consistent.

In practise, BASE has allowed for more efficient horizontal scaling at cost effective levels for systems that do not need strong consistency. The requirement to check the consistency of every single transaction at every moment of every change incurs substantial processing costs for the storage system. Eventual consistency allowed for the effective development of systems that could deal with the exponential increase of data for a variety of storage systems.

\subsubsection{Optimistic or pessimistic concurrency control}

Common to every system enabling users to execute conflicting transactions is the need to ensure that conflicting transactions are sequentially ordered to preserve storage consistency. Concurrency control mechanisms are targeted at ensuring this ordering. The two main strategies are optimistic and pessimistic concurrency control (119): 
Pessimistic concurrency control prevents the possibility of conflicting operations being applied on a data object by using locks. This strategy guarantees the changes are made safely and consistently, avoiding conflicts at all times during the processing of the transaction. The disadvantage of pessimistic locking is that a object is locked from the time it is first accessed in a transaction until the transaction is finished, making it inaccessible to other transactions during that time. If most transactions simply read from the object and never change it, an exclusive lock may be overkill as it may cause lock contention.

Optimistic concurrency control assumes that although conflicts are possible, they will be very rare. Instead of locking every object every time that it is used, the system merely looks for indications that two users actually did try to update the same object at the same time. This strategy allows conflicts to happen while a transaction is in progress, but prevents the transactions from successfully being applied should such conflict be detected upon commit.

Optimistic concurrency control is more efficient and offers higher performance when the probability of conflicts is low by avoiding unnecessary locking. Doing so, it also provides additional isolation between readers and writers. Despite these advantages, in high contention scenarios or when the duration of a transaction is high, optimistic concurrency control may cause significantly higher abort rate that ultimately hinder the performance of the application.

\subsubsection{Consensus protocols}

A common requirement for distributed transactional systems is for the sequential order between transactions to be consistent across all nodes. This requires all nodes participating in any given set of transaction to reach a common consensus on the relative ordering of these transactions. Consensus protocols have been the focus of extensive research in the past decades, leading to four main protocols:

Two-phase commit (2PC) (47) coordinates all processes that participate in a distributed transaction on if to commit or abort the transaction. It consists of two phases: vote and commit. In the first phase, each node participating in the transaction vote for the transaction to be applied or rejected. In the commit phase, 
after all nodes have voted, the transaction is applied if and only if all notes voted accordingly. A single negative vote causes the transaction to abort. In this protocol, it is critical for the node initiating the transaction to remain available. In case this node fails, some participants in a distributed system will not be able to resolve their transactions and will block.

Three-phase commit (3PC) (147) eliminates system blocking problem at the cost of latency by introducing a third phase between vote and commit: pre-commit. In this phase, the initiating node will acknowledge the results of the votes. If the initiating node fails before sending such acknowledgement, the other nodes will unanimously agree that the operation was aborted. This protocol is however not resilient to network partition. Should such partition occur during the pre-commit phase, it is possible for some nodes not to be able to converge on the outcome of the transaction.

Paxos (29) solves the partition tolerance issue by only requiring a majority of nodes to agree on the outcome of a transaction for a decision to be made. This ensures that in a given vote, if the majority of nodes has agreed upon a certain outcome, any node trying to propose a value subsequently would learn that outcome from other nodes and would agree to that outcome only. This also means that Paxos does not block even if half the nodes fail to reply. The inner complexity and generality of Paxos lead to multiple derivations being proposed in the literature (54; 94; 95 ; 96; 128). Paxos is the core of Google's Spanner (50) ACID guarantees.

Transaction chains (174) provide a faster alternative to Paxos for the case when consensus is used for storage transactions. It relies on all nodes involved in a transaction being known ahead of time by the transaction initiator and being logically ordered in a consistent way. The protocol is based on two phases: the vote and commit phases. During these phases, which are similar to those of $2 \mathrm{PC}$, a commit message is passed from node to node according to the chain order, ensuring that the commit messages of two conflicting transactions will pass through their common nodes in the same order. When the number of nodes involved in a transaction is low, this protocol yields significant performance improvement over Paxos and $3 \mathrm{PC}$ while not sacrificing partition tolerance. It is the core of the Hyperdex key-value store (61). 


\subsubsection{Limitations}

In contrast with application-specific consistency management, the transactional model transfers to the storage system the responsibility to ensure the consistency of the operations. This undoubtedly yields significant advantages. First, it provides users with a simple API enabling them to express the interdependency relation between operations without having to deal with the details behind actually enforcing this interdependency. Second, it provides consistency guarantees even in case of failures of the client application. Third, it enables storage system developers to optimize and integrate commit protocols deep in the design of the system.

Yet, transactions do not necessarily deal with all aspects of concurrency, and may not be sufficient to express highly complex relations different requests. Furthermore, although relatively fast protocols such as transaction chains exist, they incur a tangible performance penalty that has to be paid even when transaction semantics are not needed by the application. Finally, transactions take some control over the fine-grained optimization of concurrency management out of the hands of the developer, offering fewer potential for optimization.

\subsection{CAP: Consistency, Availability, Partition tolerance}

The consistency model adopted by the storage system itself has implications that go beyond the sole data consistency guarantees. Indeed, the CAP theorem (35) conjectures that in any networked shared-data system there is a fundamental trade-off between consistency, availability, and partition tolerance. The theorem states that such systems can only guarantee two of the following three properties:

Mutual Consistency: Every node in a distributed cluster returns the same, most recent, successful write. The returned results are always mutually consistent, i.e. they represent a logical state of the system. The consistency model used in the proof of the CAP theorem is sequential consistency.

Availability: Every non-failing node returns a response for all read and write requests in a reasonable amount of time. 
Partition Tolerance: The system continues to function and upholds its consistency guarantees in spite of network partitions. Distributed systems guaranteeing partition tolerance can gracefully recover from partitions once the partition heals.

In this big picture, most distributed file systems are usually CP (they combine Strong consistency with Partition Tolerance). In contrast, key-value stores and object stores are either CP or AP (Availability with Partition Tolerance) depending on whether they offer strong or weak consistency.

It is worth to note that CA (Availability with Strong consistency) is often not a meaningful choice for distributed systems. Indeed, the potential to have partitions is one of the definitions of a distributed system. In case of a CA system, ignoring network partitions automatically forfeits $\mathrm{C}, \mathrm{A}$, or both depending on whether the system corrupts data or crashes when experiencing an unexpected partition.

However, the failure model used in the CAP theorem is unconstrained; it only considers an omnipotent adversary that can induce any number of faults into the system. Under such an assumption, no system can remain available or consistent. By making a stronger assumption that limits failures to affect at most a threshold of servers, it is possible to develop systems offering both consistency, availability and partition tolerance as long as this failure threshold is not reached. This is most notably the case of HyperDex Warp (60; 61) and Google's Spanner (50).

In the context of HPC and BDA convergence, neither availability nor partition tolerance can be forfeited. Thus the question is how to adjust the consistency setting to answer the precise needs of the application without sacrificing performance.

\subsection{Conclusions: which consistency model for converging storage?}

We can observe that the storage systems used for HPC tend to offer strong consistency. In contrast, BDA relies on a variety of systems targeted at different use-cases, each offering different consistency models ranging from BASE to full ACID. While both application-specific and transactional concurrency management each have advantages of their own, it is clear that an application that does not require transactions can be executed on a transactional system, while the opposite is not possible or would require 
significant amount of work. In the end, transactional systems offer a versatile and generic solution to handling consistency in a wide range of application. Indeed, it can at the same time meet the needs of legacy HPC and BDA applications expecting strong consistency and those of BDA applications accepting either strong consistency level or lesser guarantees. 


\section{Part II}

\section{Týr: a transactional, scalable data storage system}





\section{Chapter 5}

\section{Blobs as storage model for convergence}

State-of-the-art storage systems for both HPC and BDA show a common trend towards relaxing many of the concurrent file access semantics, trading such strong guarantees for increased performance. Nevertheless, some differences remain. Specifically, while the BDA community increasingly relies on systems dropping the POSIX I/O API altogether, the HPC community tends to provide this relaxed set of semantics behind the same API. Although this choice increases backwards compatibility with legacy applications, it also has significant performance implications due to the design constraints this standard imposes, i.e. hierarchical namespace or permission management.

In this chapter, we propose and discuss blobs as an alternative to traditional distributed file systems. Inspired by the API similarity with distributed file systems, allowing for random reads and writes in binary objects, we analyze the benefits and limitations of blobs as a storage model for HPC and BDA convergence.

\subsection{General overview, intuition and methodology}

Very few HPC applications actually rely on the full POSIX I/O API. The MPI-IO standard does not only relax many of the associated semantics but also drops many of its operations altogether (97; 132). For example, it does not expose the file hierarchy or permissions to the end user. Therefore, applications leveraging these libraries are not supposed to expect these features to be provided. 
The same observation can be drawn for BDA applications. Hadoop (145), Spark (173) or Flink (41), the three most popular BDA frameworks, are all able to leverage the HDFS file system. Yet, neither these frameworks do expose the hierarchical nature of the underlying storage to the application. Furthermore, these frameworks are also able to leverage wide column stores which lack a hierarchical structure entirely (56, 164).

In this chapter we show experimentally that blobs can indeed substitute traditional file systems for both HPC and BDA workloads. We demonstrate that the hierarchical nature of distributed file systems is only provided for convenience to the end-user, and is only seldom exploited by the applications, if ever. The consequence would be the majority of storage operations to be file operations (open, close, read, write, create, delete). These operations are very close to the ones proposed by blob storage systems. Directory-level operations (opedir, mkdir, rmdir) do not have their blob counterpart because of the flat nature of the blob namespace. Should applications really need them (e.g., legacy applications), such operations can be emulated using a full database scan. This implementation would intuitively not be optimized compared to its counterpart on current file systems. Yet, since we expect these calls to be vastly outnumbered by blob-level operations, this performance penalty is likely to be compensated by the gains permitted by using a flat namespace and simpler semantics.

\subsubsection{Applications}

The challenges posed by convergence between HPC and BDA applications have raised many discussions in the community (6). One of the emerging ideas from these discussions is that the applications cannot be considered separately from the underlying software stack; the fuel for convergence could be a wide variety of HPC and BDA applications leveraging converged services and underlying infrastructure. Although designing a full converging stack is not the objective of this thesis, we focus on storage as one of the critical milestones that could make such convergence possible. We choose I/O-intensive applications, which could benefit most from such converged storage.

We base our experiments on a number of I/O-intensive applications extracted from the literature (64; 103; 166) that cover the diversity of I/O workloads commonly encountered on both HPC and BDA platforms. 


\subsubsection{HPC Applications}

The HPC applications we use are based on MPI. They all leverage large input or output datasets associated with large-scale computations atop centralized storage usually provided by a distributed, POSIX-IO-compliant file system such as Lustre (142).

mpiBLAST (51) is a parallel MPI implementation of NCBI BLAST (171). It is a readintensive biomolecular tool targeted at searching for regions of similarity between biological sequences. The program compares nucleotide or protein sequences to sequence databases and calculates the statistical significance.

MOM (Modular Ocean Model) (71) is a read-intensive three-dimensional ocean circulation model targeted at understanding the ocean climate system. It is developed at the NOAA's Geophysical Fluid Dynamics Laboratory.

ECOHAM5 (ECOlogical model, HAMburg, version 5) (149, 150) is a write-intensive three-dimensional biogeochemical ecosystem model with the focus on the North Sea (73; 108). It modelizes the pelagic and benthic cycles of carbon, nitrogen, phosphorus, silicon and oxygen on the northwest European continental shelf. It was developed at the Hamburg Institute of Oceanography.

Ray Tracing is a balanced read-write workload extracted from the BigDataBench (166), itself derived from (48; 152). It uses scene description files to generates images by tracing the path of light and simulating the effects of its encounters with virtual objects.

\subsubsection{BDA Applications}

As the leading open-source Big Data processing and analytics framework, Apache Spark (173) appears as an ideal candidate for this research. SparkBench (17; 103) is a widely-recognized, industry-standard benchmarking suite for Spark. It comprises a representative set of workloads belonging to four application types: machine learning, graph processing, streaming, and SQL queries. We extract the test applications from this tool, focusing on data-intensive ones:

Sort is a widely used benchmark that reads input data and sorts it based on a given key. It is I/O-intensive since all the data read will be processed and written back 
to the file system. For example, it can be used to sort a series of readings from sensors by date.

Grep is a filtering workload that searches in the input data for lines containing a given word and saves these lines into HDFS. In contrast to Sort, the size of the input and output will not be equal, and some data will be filtered out. It can be used to extract all the documents that contain a word of interest in a huge corpus.

Decision Tree is a machine learning workload that reads a dataset containing rows with a series of features and a class they belong to. This dataset is then split into a training and a test set. The workload creates a predictive model with the training set that is able to predict the class of the elements in the test set. These predictions are written back to disk. An example could be classifying data packets into malicious or nonmalicious activity.

Connected Component is an algorithm that finds the subgraphs in a graph in which any two vertices are connected by a path but are not connected to any other node on the supergraph. This can be seen as a way of finding clusters of nodes. For example, in a social network it can be used to find communities of users. The implementation in the benchmark will read a dataset and write the labels of each component back to disk.

Tokenizer is a Spark application we speci

cally developed to add. It reads a text file, tokenizes each line into words, and calculates the NGrams=2 for each line. These Ngrams are saved in a text file. This is a common preprocessing step in topic modeling for documents where word patterns have to be extracted as an input to a machine learning model.

\subsubsection{Summary}

In Table 5.1 we provide a summary of the applications we leverage for our experiments in this chapter. For each application we provide the read and written data sizes, as well as the resulting application profile. 


\begin{tabular}{|l|l|l|l|l|l|}
\hline & Application & Usage & Reads & Writes & Profile \\
\hline \hline \multirow{4}{*}{ HPC } & mpiBLAST (BLAST) & Protein docking & $27.7 \mathrm{~GB}$ & $12.8 \mathrm{MB}$ & Read-intensive \\
\cline { 2 - 6 } & MOM & Oceanic model & $19.5 \mathrm{~GB}$ & $3.2 \mathrm{~GB}$ & Read-intensive \\
\cline { 2 - 6 } & ECOHAM (EH) & Oceanic model & $0.4 \mathrm{~GB}$ & $9.7 \mathrm{~GB}$ & Write-intensive \\
\cline { 2 - 6 } & Ray Tracing (RT) & Video processing & $67.4 \mathrm{~GB}$ & $71.2 \mathrm{~GB}$ & Balanced \\
\hline \hline \multirow{3}{*}{ BDA } & Sort & Text Processing & $5.8 \mathrm{~GB}$ & $5.8 \mathrm{~GB}$ & Balanced \\
\cline { 2 - 6 } & Connected Component (CC) & Graph Processing & $13.1 \mathrm{~GB}$ & $71.2 \mathrm{MB}$ & Read-intensive \\
\cline { 2 - 6 } & Grep & Text Processing & $55.8 \mathrm{~GB}$ & $863.8 \mathrm{MB}$ & Read-intensive \\
\cline { 2 - 6 } & Decision Tree (DT) & Machine Learning & $59.1 \mathrm{~GB}$ & $4.7 \mathrm{~GB}$ & Read-intensive \\
\cline { 2 - 6 } & Tokenizer & Text Processing & $55.8 \mathrm{~GB}$ & $235.7 \mathrm{~GB}$ & Write-intensive \\
\hline
\end{tabular}

Table 5.1: HPC and BDA application summary

\subsubsection{Experimental platform}

We run experiments on the Grid'5000 (25) experimental testbed, which spans 11 sites in France and Luxembourg. For these experiments, the parapluie cluster of Rennes was used. Each node embeds 2 x 12-core $1.7 \mathrm{Ghz} 6164 \mathrm{HE}, 48 \mathrm{~GB}$ of RAM, and 250 GB HDD. Network connectivity is supported either with Gigabit Ethernet connectivity (MTU $=1500 \mathrm{~B}$ ) or by $4 \times 20 \mathrm{G}$ DDR InfiniBand. We use the former for BDA and the latter for HPC applications in order to fit with the usual configuration of each platform.

HPC applications ran atop Lustre 2.9.0 and MPICH 3.2 (72), on a 32-node cluster that we configure with multiple ratios of storage-to-compute nodes. Networking is provided on each node with 4 x 20G DDR InfiniBand. BDA application ran atop Spark 2.1.0, Hadoop / HDFS 2.7.3 and Ceph Kraken using Gigabit Ethernet connectivity. The cluster is composed of the same 32 nodes.

\subsection{Storage call distribution for HPC and BDA applica- tions}

In this section we demonstrate that the actual I/O calls made by both HPC and BDA applications are not incompatible with the set of features provided by state-of-the-art blob storage systems. Our intuition is that read and write calls are vastly predominant in the workloads of those applications and that other features of distributed file systems such as directory listings are rarely used, if at all. 


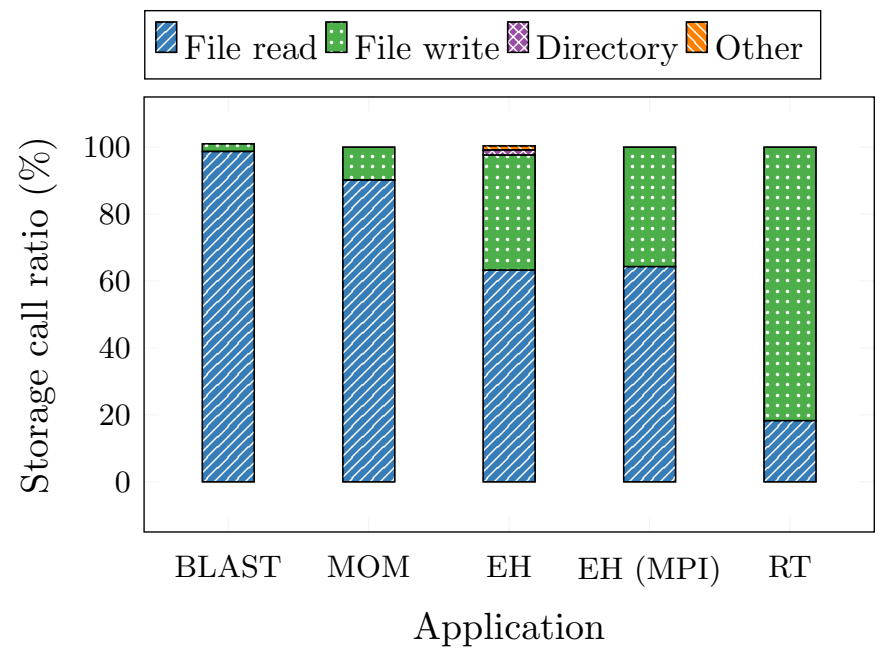

Figure 5.1: Measured relative amount of different storage calls to the persistent file system for HPC applications

\subsubsection{Tracing HPC applications}

Figure 5.1 summarizes the relative count of storage calls performed by our set of HPC applications. The most important observation for all four applications is the predominance of reads and writes. Except for ECOHAM, no application performed any other call to the storage system that reads or writes files, thus confirming our first intuition. This was expected because the MPI-IO standard does not permit any other operation.

The few storage calls other than read and write (mainly extended attributes reads and directory listings) are due to the run script necessary to prepare the run and collect results after it finishes. These steps can be offlined from the I/O-heavy MPI part of the application. This results in only reads and writes being performed (EH / MPI).

We conclude that the only operations performed by our set of HPC applications, namely, file I/O, can be mapped to blob I/O on a blob storage system. Consequently, these applications appear to be suited to run unmodified atop blob storage.

\subsubsection{Tracing BDA applications}

Figure 5.2 shows the relative count of storage calls performed by our set of BDA applications to HDFS. Similar to what we observed with HPC applications, the storage calls are vastly dominated by reads and writes to files. In contrast with HPC, however, all applications also cause Spark to perform a handful of directory operations (91 in total across all our applications out of over 10 million individual storage operations in 


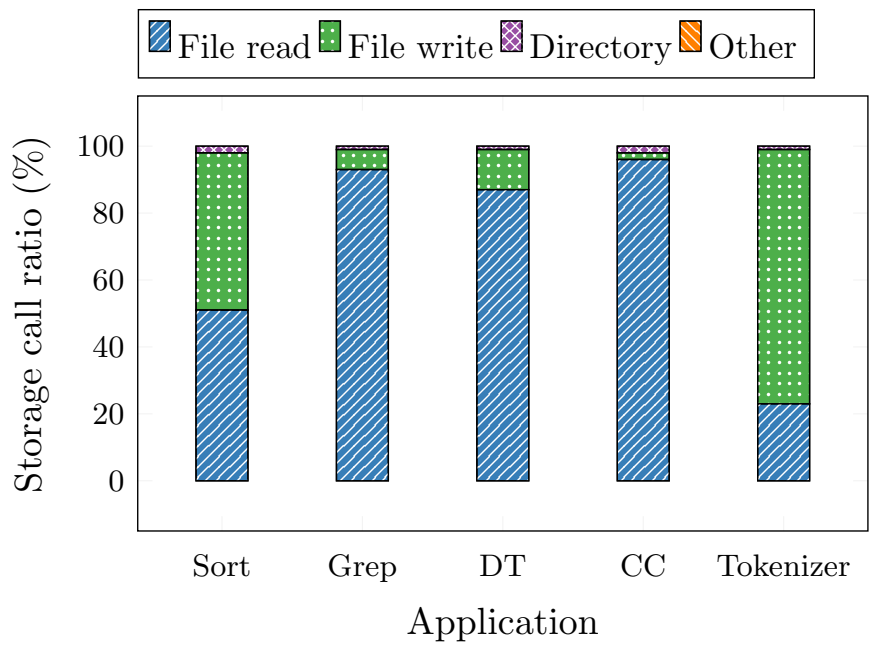

Figure 5.2: Measured relative amount of different storage calls to the persistent file system for BDA applications

\begin{tabular}{|c|c|c|}
\hline Operation & Action & Count \\
\hline mkdir & Create directory & 43 \\
\hline rmdir & Remove directory & 43 \\
\hline opendir (Input directory) & $\begin{array}{c}\text { Open / List } \\
\text { directory }\end{array}$ & 5 \\
\hline opendir (Other directories) & $\begin{array}{c}\text { Open / List } \\
\text { directory }\end{array}$ & 0 \\
\hline
\end{tabular}

Table 5.2: Spark directory operation breakdown. For comparison, all applications performed over 10 million total individual storage operations during this benchmark.

total). These directory operations are not related to the data processing because input / output files are accessed directly by using read and write calls.

Analyzing these directory operations, we note that they are related solely to $(i)$ creating the directories necessary to maintain the logs of the application execution, (ii) listing the input files before each application runs if the input data is set as a directory, and (iii) maintaining the .sparkStaging directory. This directory is internally used by Spark to share information related to the application between nodes and is filled during the application submission. It contains application files such as the Spark and application archives, as well as distributed cache files (11).

We analyze in detail the directory operations performed by BDA applications. Table 5.2 shows the breakdown of all such directory operations across all applications by storage call. We note that only the input data directories are listed, meaning that Spark accesses directly all the other files it needs with their path. Consequently, a flat 
namespace such as the one provided by blob storage systems could probably be used.

\subsection{Replacing file-based by blob-based storage}

In this section we demonstrate the potential of blob-based storage to suit the storage needs of both HPC and Big Data applications. To do so, we deploy each aforementioned application atop two state-of-the-art blob storage systems. We prove that the performance of these applications running atop converged blob-based storage matches or exceeds that of the same applications running atop Lustre (142) for HPC, as well as Ceph (167) and HDFS (145) for Big Data.

\subsubsection{Overview of the blob storage systems}

We run our applications atop two state-of-the-art blob storage systems: BlobSeer (127) and Rados (169). We are using only the basic blob storage functionality they provide, and do not make use of any advanced features they may support. Although their highlevel design has similarities, these two systems have different strengths and weaknesses resulting from design decisions made for each to support specific use cases.

Rados is the storage layer for Ceph. Rados has the ability to scale to thousands of hardware devices by using management software that runs on each of the individual storage nodes. The software provides features such as thin provisioning, snapshots and replication.

BlobSeer is an open-source, distributed storage that offers a similar interface to that of Rados. It is specifically designed for highly-concurrent access patterns. Its architecture is based on Multi-Version Concurrency Control (MVCC), associated with a decentralized metadata management based on a distributed tree. It supports atomic snapshots and replication.

We assess the performance impact of replacing file-based with blob-based storage by observing three metrics.

- The job completion time is the total execution time of the application, from submission to completion. 


\begin{tabular}{|c|c|}
\hline POSIX Call & Translated Call \\
\hline create(/foo/bar) & create(/foo_bar) \\
\hline open(/foo/bar) & open(/foo__bar) \\
\hline read(fd) & read(bd) \\
\hline write(fd) & write(bd) \\
\hline mkdir(/foo) & Unsupported operation \\
\hline opendir(/foo) & Unsupported operation \\
\hline rmdir(/foo) & Unsupported operation \\
\hline
\end{tabular}

Table 5.3: HPC storage call translation rules on a flat namespace

- The read bandwidth and write bandwidth respectively represent the average data transferred per unit of time for read and write requests.

We collect all these metrics on the compute nodes by instrumenting the storage systems, and we aggregate the results. All the storage systems leveraged in these experiments are in a similar fashion. Specifically, all systems use a stripe size of $64 \mathrm{MB}$ as well as a replication factor of 3 .

\subsubsection{Replacing Lustre with blob-based storage on HPC}

In this section, we demonstrate how blob-based storage system can be used to transparently support HPC applications while matching or exceeding Lustre I/O performance by replacing the latter with both BlobSeer and Rados. We experiment using three storage-to-compute node configurations in order to ensure that our results are independent of the cluster configuration. We run the same experiments respectively with 28 compute / 4 storage nodes, $24 / 8$ and $20 / 12$. We average the results of 100 experiment runs.

On each node, we deploy a small interceptor to redirect POSIX storage calls to the blob storage system. It is based on FUSE (153), which is supported on most Linux kernels today. This adapter translates file operations to blob operations according to Table 5.3. Directory operations are not supported as we showed previously that they are unnecessary for HPC applications. The APIs of the blob storage systems we consider allow for a direct mapping between file-based and blob-based storage operations. We partially implement the stat function. Specifically, the file size is mapped to the blob size, the permissions are set to 777 , the block size and allocated block size are set to 
$\square$ Lustre百BlobSeer Rados

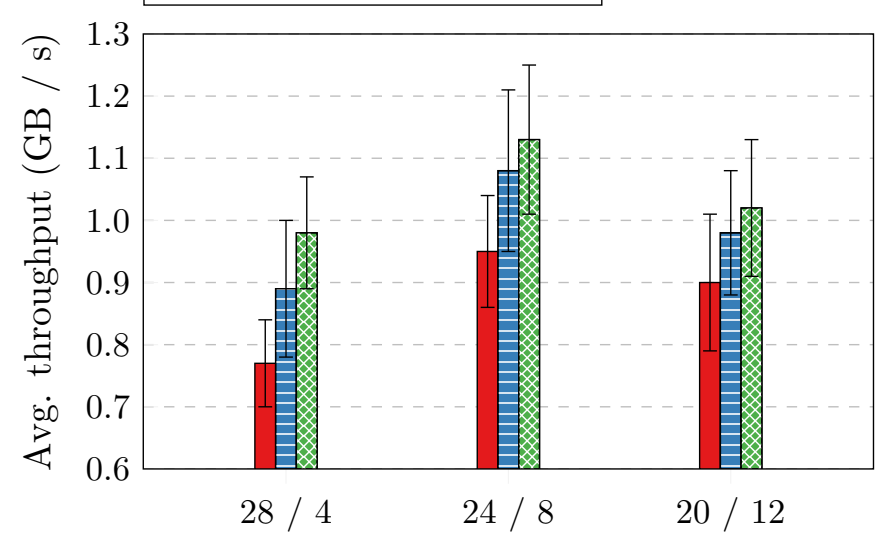

Compute nodes / Storage nodes

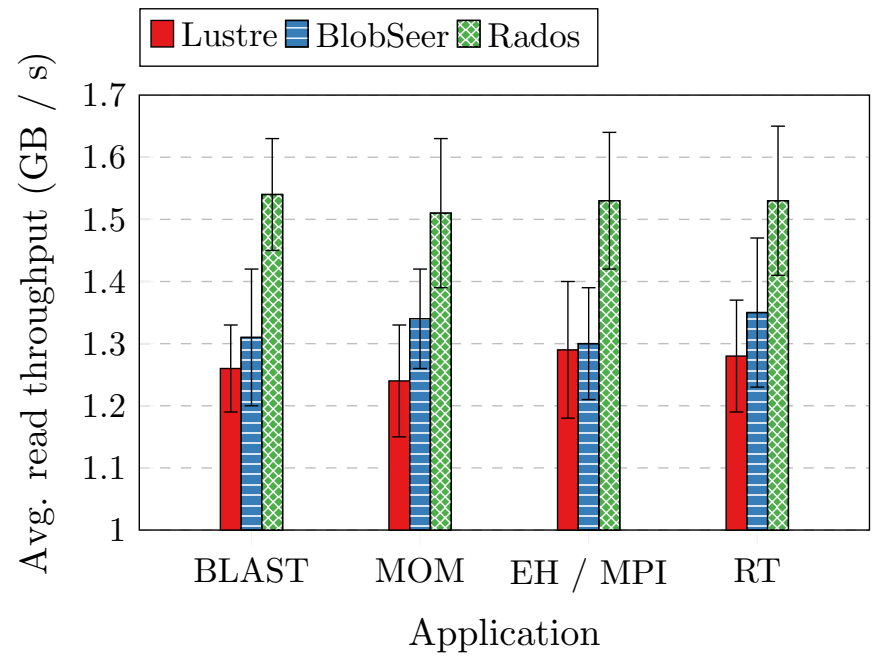

Figure 5.3: Average agreggate throughput across all HPC applications varying the compute-to-storage ratio, with $95 \%$ confidence intervals.

Figure 5.4: Comparison of read throughput for each HPC application with Lustre, BlobSeer and Rados, with $95 \%$ confidence intervals.

512 bytes, and the inode number is set to the hash of the blob key. The remaining information is set to 0 . Our implementation does not support symbolic or hard links, which are not needed by our applications.

In Figure 5.3 we plot the average aggregate read and write bandwidth for all applications while varying the compute-to-storage node ratio. We note that for our configuration the 24 compute node / 8 storage node setup results in the highest bandwidth for all storage systems. Hence, the following experiments are performed with that configuration. This ratio is much lower than on common HPC platforms (3:1 vs. $\sim 70: 1$ at ORNL, for instance (144)) mainly because the jobs we run are significantly more dataintensive than compute-intensive. We note from these results that blob storage systems 


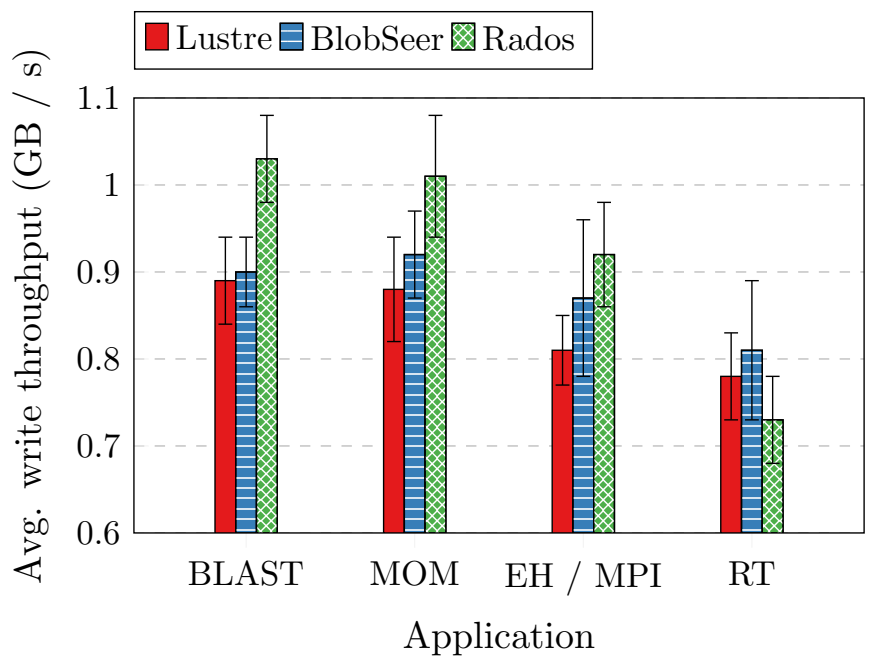

Figure 5.5: Comparison of write throughput for each HPC application with Lustre, BlobSeer, and Rados, with $95 \%$ confidence.

Figure 5.6: Average performance improvement relative to Lustre for HPC applications using blob-based storage, with $95 \%$ confidence.

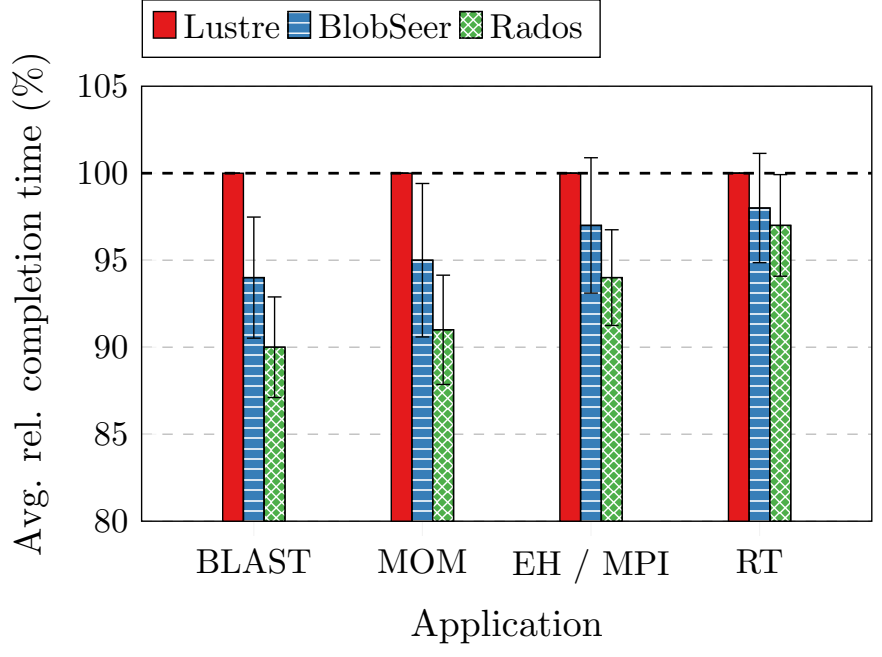

constantly outperform Lustre in all configurations for both reads and writes. We will detail these results in the following experiments. For read-intensive applications such as BLAST and MOM, this performance increase allows blob storage systems with 4 storage nodes to achieve a bandwidth comparable to Lustre's with 8 storage nodes.

In Figure 5.4 we plot the average read bandwidth for each of our HPC applications with Lustre file-based storage and BlobSeer or Rados blob-based storage. We note an average $14 \%$ reduction of the total read time when using Rados storage compared to Lustre. This is because unlike Lustre and BlobSeer, Rados enable clients to locate any piece of data without prior communication with any dedicated metadata node.

We plot in Figure 5.5 the average write bandwidth obtained in similar conditions. 
Although these results show that blob storage systems still outperform Lustre in all cases except for Ray Tracing in which Rados is outperformed by Lustre. However, the performance gain is lower, at $6 \%$ on average. The result for Ray Tracing is explained by the particular access patterns, which include concurrent write accesses to files. This pattern is unfavourable for systems leveraging lock-based concurrency management such as Lustre and Rados. BlobSeer multi-version concurrency control excels in that case. We observe that BlobSeer outperforms Rados in write performance for Ray Tracing, in contrast to the trend we observe with other applications. This is because this application tends to perform smaller writes. Lustre and Týr are both efficient at this type of workloads. In the case of Týr, this is largely due to the co-location of the version metadata with the first data chunk of the blobs.

In Figure 5.6 we plot the average application completion time improvement. The I/O performance gains are here diluted in compute operations. As expected considering the previous results, read-intensive applications exhibit the greatest decrease. BLAST and MOM show a completion time reduction of nearly $8 \%$ with both blob storage systems. In contrast, write-intensive applications such as ray tracing show a lower $3 \%$ completion time decrease with BlobSeer or Rados as the underlying storage when compared with Lustre.

\subsubsection{Running BDA applications atop blob storage}

In this section we run the same set of experiments for the set of Big Data applications. We demonstrate that BlobSeer and Rados significantly outperform HDFS for all applications. In order to provide an additional baseline of the performance of file systems, we also run these applications atop the Ceph (167) file system, itself based on Rados.

We use the same configuration as in Section 5.2, running computation alongside storage on 32 nodes. We integrate the storage adapter for blob storage directly inside HDFS. The Hadoop installation has been modified to redirect storage calls to blob storage systems. The translation between POSIX-like calls and flat-namespace blob operations is done using the translation rules defined in Table 5.4. We maps all file operations directly to their blob counterpart. Directory operations are simulated using namespace scans. Despite being very costly, the low number of such calls relative to file operations should not significantly impact the application performance when the number of files is low. We implement the CRUSH algorithm (168) to provide Spark 


\begin{tabular}{|c|c|}
\hline $\begin{array}{l}\text { Original } \\
\text { operation }\end{array}$ & Rewritten operation \\
\hline create(/foo/bar) & create(/foo__bar) \\
\hline open (/foo/bar) & open(/foo__bar) \\
\hline $\operatorname{read}(f d)$ & $\operatorname{read}(\mathrm{bd})$ \\
\hline write (fd) & write (bd) \\
\hline $\operatorname{mkdir}(/ f \circ o)$ & Dropped operation \\
\hline opendir (/foo) & $\begin{array}{c}\operatorname{scan}(), \text { return all like } \\
\text { /foo_-* }\end{array}$ \\
\hline rmdir (/foo) & $\begin{array}{c}\operatorname{scan}(), \text { remove all like } \\
/ \text { foo_** }\end{array}$ \\
\hline
\end{tabular}

Table 5.4: BDA storage call translation rules on a flat namespace

with the physical data location on Rados and Ceph. We use the client API capabilities to provide that information in BlobSeer.

In Figure 5.7 we plot the average read bandwidth achieved for SparkBench. While Rados significantly outperforms all systems for all applications, we note an overall performance tie between BlobSeer and Ceph. This is because of the heavy metadata management in all cases, which heavily impacts access latency and consequently read bandwidth. The performance cost of file-based storage is highlighted by the performance difference between Ceph and Rados, upon which it is based. As with HDFS, this is mostly due to the additional communication required with dedicated metadata servers in the critical path for read requests, necessary for the hierarchy management.

Figure 5.8 shows the average write bandwidth for these applications. Similar to what was observed with HPC, we note a constant write bandwidth improvement with blob storage over HDFS and Ceph. We also note the same pattern we observed with HPC. Specifically, Rados outperforms BlobSeer on read-intensive applications, whereas BlobSeer enables higher throughput on write-intensive applications. This is visible with the Tokenizer application, where lock contention due to lack of multiversion concurrency control in Rados causes significant performance loss on concurrent write access.

In Figure 5.9 we plot the relative improvement in the total application completion time, diluted in computation. Running Big Data applications atop blobs improves application completion time, up to $22 \%$ compared to HDFS and $7 \%$ compared to Ceph. 


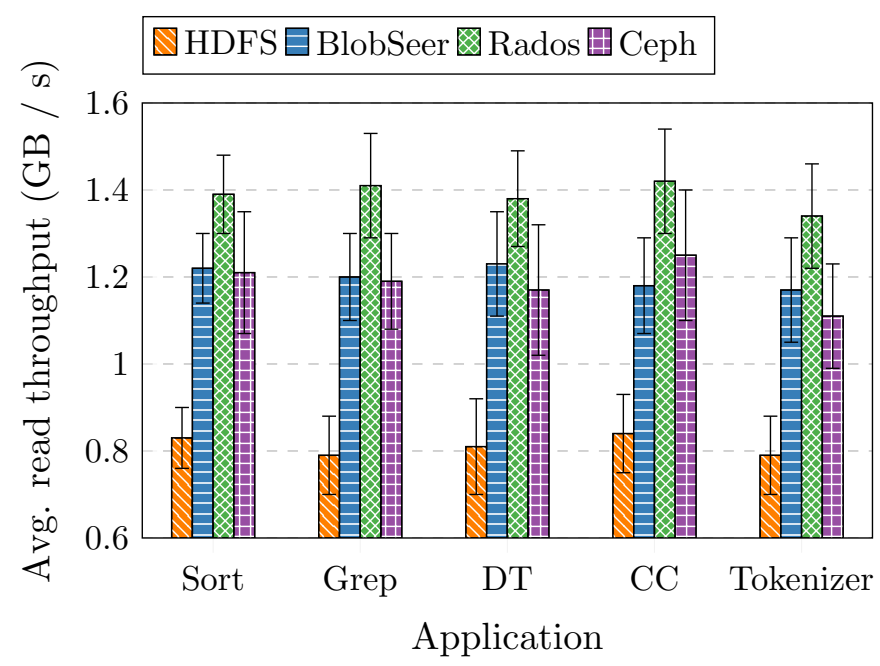

Figure 5.7: Comparison of read throughput for each Big Data application with HDFS, BlobSeer, Rados and CephFS, with 95\% confidence intervals.

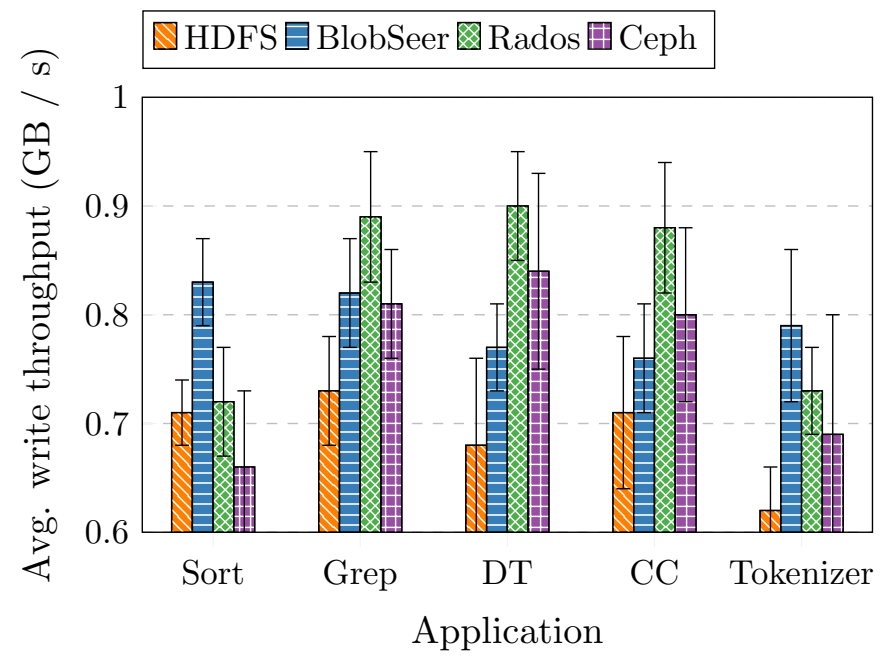

Figure 5.8: Comparison of write throughput for each Big Data application with HDFS, BlobSeer, Rados and CephFS, with 95\% confidence intervals.

For Big Data, the highest gains are obtained with read-intensive applications such as Grep and Decision Tree. In comparison, write-intensive applications such as Tokenizer also benefit from improved performance, although relatively smaller due to the globally greater complexity of the write protocols for each storage system.

\subsection{Conclusion: blob is the right storage paradigm for convergence}

In this chapter, we obtained important results that confirm the applicability of blobs for HPC and BDA convergence. First, the set of applications from both HPC and 


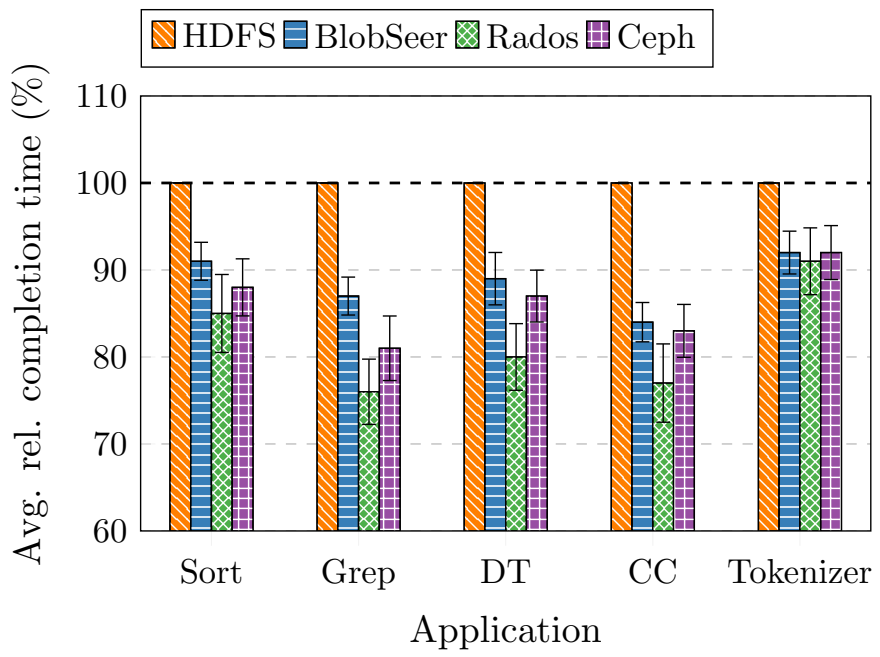

Figure 5.9: Average performance improvement relative to HDFS for Big Data applications using blob-based storage, with $95 \%$ confidence intervals.

BDA communities almost exclusively perform file-level operations. These operations are very close to those provided by blob storage. Second, replacing file-based with blobbased storage does not hinder the performance of the applications. On the contrary, a performance gain of up to $32 \%$ is observed compared to file systems, which is mostly explained due to the optimized read path the flat namespace permits. In particular, the direct read capability and simple, decentralized metadata management scheme that Rados provides excels for read performance, while the multi-version concurrency control of BlobSeer supports high write velocity for highly concurrent workloads.

These results prove that for both HPC and BDA use-cases, blobs are able to support legacy applications at high velocity. This make them ideal candidates for storage-based convergence between HPC and BDA. 


\section{Chapter 6}

\section{Týr design principles and architecture}

In the previous chapter we factually demonstrated that blobs are a strong candidate for answering the storage needs of both legacy HPC and BDA applications. Yet, these experiments also highlighted the limitations of state-of-the-art systems for such usecases. While Rados performance is excellent under low write contention, its lockbased concurrency control limits its throughput in highly-concurrent use-cases. On the contrary, the multi-version concurrency control of BlobSeer supports its performance under high concurrency, but the distributed metadata tree that is core to its design induces a significant read latency that hinders application performance.

In addition, while these blob systems answer the needs of legacy applications, a range of advanced use-cases for BDA applications require stronger consistency semantics, ensuring the correctness of multi-objects operations. This is notably the case of data indexing and aggregation (Chapter 9), or the synchronization of application data and metadata (Chapter 12). The latter use-case is illustrated by the design of the Warp Transactional Filesystem (WTF) (59) relying on transactions for metadata operations, or the reliance of HBase (65) on ZooKeeper (80) for synchronization purposes.

In this chapter, we propose the design of Týr, a blob storage system designed from the ground up to support multi-object synchronization with transactional semantics. Týr notably combines predictable data distribution that is central to the low-latency of Rados with the multi-version concurrency control that is key to the high-performance of BlobSeer under high write contention scenarios. 


\subsection{Key design principles}

In this section, we summarize the key design principles that lay the foundation of Týr, in support of HPC and BDA convergence. Its key features are:

Predictable data distribution. Similarly with Rados (169) as well as a number of key-value stores (55, 86, 148), Týr enables clients to access any piece of data without any prior communication with any metadata node. This ensures low read and write latency by eliminating remote fetches as happens in the case of BlobSeer (127). We detail this feature in Section 6.2 .

Transparent multi-version concurrency control. Multi-version concurrency control isolates readers from concurrent writers. Essentially, it enables writers to modify a copy of the original data while enabling concurrent readers to access the most recent version. Each user connected to the database sees a snapshot of the database at a particular instant in time. Any changes made by a writer will not be seen by other clients until the changes have been completed. This approach proves critical to the performance of BlobSeer under high contention. Contrary to BlobSeer, Týr does not expose multiple versions to the reader, keeping this complexity hidden from the clients. We detail version management in Section 6.3

ACID transactional semantics. Týr offers built-in ACID transactions as a way to ensure the consistency and correctness of multi-object operations. The lightweight transactional protocol is core to the internals of Týr. It notably ensures Týr linearizability, and supports its replication-based fault-tolerance as well as the internal version garbage collection. We detail Týr's transactional semantics in Section 6.4 .

Atomic transform operations. Atomic transform operations enable users to perform simple binary data manipulation in-place, with a single round-trip between the client and the storage cluster. This includes binary additions, multiplications or bitwise operations. Such operations are key to efficient data aggregation (Chapter 9). They are supported by the internal transactional protocol of Týr. We detail these operations in Section 6.5. 


\subsection{Predictable data distribution}

The data are distributed in the cluster using a combination of consistent hashing and data striping techniques.

Consistent hashing (83) is a well known technique to distribute data in a cluster. The unique key associated with each object is hashed with a function that is common to all nodes in the cluster. The hashed value is used to determine the server which is responsible for the data with this key. Each node in the cluster is responsible for a part of the whole hash value range. The benefit of this technique is twofold. First, it distributes the loads across the whole cluster and helps alleviate potential hot spots when the number of objects is high enough. Second, it obviates the need for a centralized metadata server. Indeed, any node in the cluster is able to locate any piece of data with only knowledge of the mapping between nodes and hash value ranges. Furthermore, when this mapping is shared with the clients, they are able to send requests directly to the nodes holding the value they seek to read or write. This consequently eliminates unnecessary hops for pinpointing the location of data in the cluster. Such feature is key to the low-latency of key-value stores such as Chord (151), Dynamo (55) or Riak (86).

Data striping complements this data distribution. Each blob in the system is split into chunks of fixed size, that are distributed over all nodes of the system using consistent hashing. Thereby, the I/O is distributed over a larger number of nodes, supporting the scalability and performance of the system. This principle also enables storing objects with a size greater than the capacity of a single machine. With a chunk size $s$, the first chunk $c_{1}$ of a blob will contain the bytes in the range $[0, s)$, the second chunk $c_{2}$, possibly stored on another node, will contain the bytes in the range $[s, 2 s)$, and the chunk $c_{n}$ will contain the bytes in the range $[(n-1) * s, n * s)$. The size of the chunks being distributed in the cluster using consistent hashing, their size must be fixed.

Chunks are distributed in the cluster as follows. Given a hash function $h(x)$, the output range $\left[h_{\min }, h_{\max }\right]$ of the function is treated as a circular space, or ring $\left(h_{\min }\right.$ sticking around to $\left.h_{\max }\right)$. Each node is assigned a different random value within this range, which represents its position on the ring. For any given chunk $n$ of a blob $k$, 
a position on the ring is calculated by hashing the concatenation of $k$ and $n$ using $h(k: n)$. The primary node holding the data for a chunk is the first one encountered while walking the ring passed this position. Additional replicas are stored on servers determined by continuing walking the ring until the proper number of nodes is found.

\subsection{Transparent multi-version concurrency control}

Versioning is the core principle we use for data management; a new data version is generated each time a blob is written to. This yields the following advantages:

High troughput under heavy access concurrency. Compared to systems where locks are used to synchronize concurrent access to a given piece of data, versioning offers significant performance advantages. Once written, a data version is considered immutable. As such, the data is never overwritten; instead, writes operate on a copy of the current version instead of overwriting it. The main benefit of such approach is that readers are not impacted by concurrent writers, and do not need to wait for conflicting write operations to finish. This contrasts with lock-based storage systems such as Rados, which typically offer degraded read throughput under heavy access concurrency because of lock contention.

Enhanced fault tolerance. A significant challenge when data is not versioned is the data recovery in the presence of failures. Indeed, should a fault occur during a write, the data could be left in an unconsistent state, hence rendering data recovery difficult. In contrast, since versioning ensures that writes only operate on a copy of the data, the previous version can be restored and the incomplete write discarded.

We propose to handle versioning at the chunk level, in contrast with traditional implementations of multi-version concurrency control. This obviates the need for centrally generating sequential version numbers, which are normally used to determine the ordering of successive versions. Generating such successive version numbers in a distributed context is a difficult problem; the common solutions either rely on a heavyweight consensus algorithm such as Paxos (95) or come at the cost of fault tolerance when a single sequencer is used as in BlobSeer. 

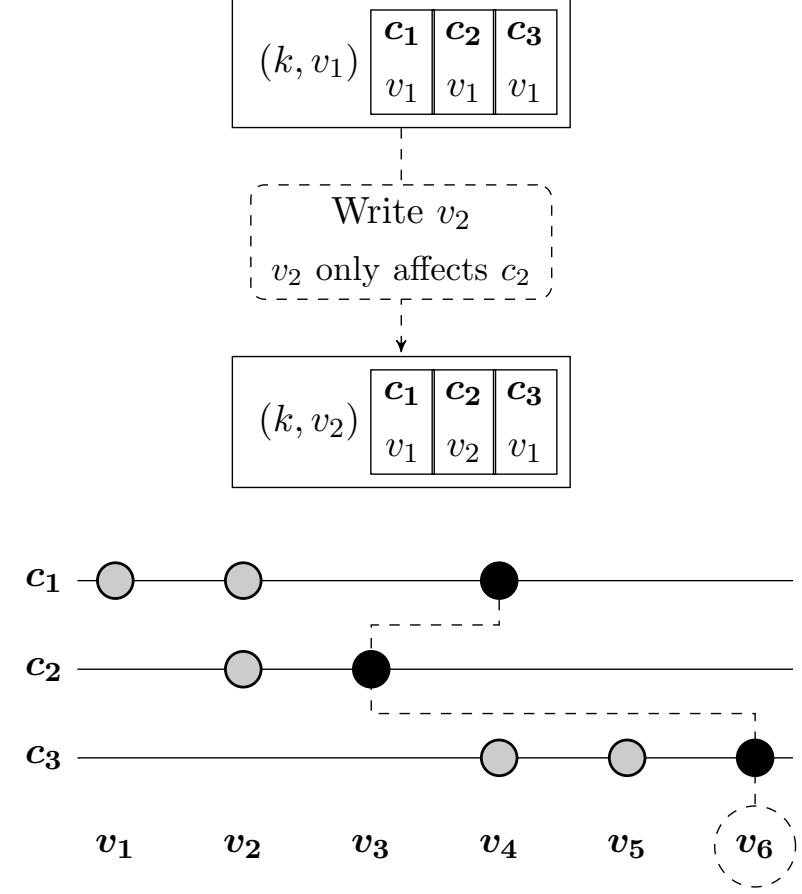

Figure 6.1: Týr versioning model. When a version $v_{2}$ of the blob is written, which only affects chunk $c_{2}$, only the version of both the blob and $c_{2}$ is changed. The version id for both $c_{1}$ and $c_{3}$ remains unchanged. Sequential version numbers are used for simplicity.

Figure 6.2: Version management example. The version $v_{1}$ of this blob only affected the chunk $c_{1}, v_{2}$ affected both $c_{1}$ and $c_{2}$. In this example, $v_{6}$ is composed of the chunk versions $\left(v_{4}, v_{3}, v_{6}\right)$. This versioning information is stored on the blob's metadata nodes. Sequential version numbers are used for simplicity.

In contrast, we propose to use non-sequential numbers for versioning. The ordering of successive versions of any given chunk is retained at each node storing a replica of this chunk. The blob version identifier is the same as the most recent version identifier of its chunks, as illustrated in Figure 6.1

For any given write operation, only the nodes holding affected data chunks will receive information regarding this new version. Consequently, the latest version of a blob is composed of a set of chunks with possibly different version identifiers, as illustrated by Figure 6.2. To be able to read a consistent version of any given blob, information regarding every successive versions of all chunks composing a blob is stored on the same nodes holding replicas of the first data chunk of the blob. These nodes are called version managers for the blob. Co-locating the first chunk of a blob and its version managers enables faster writes to the beginning of blobs. It also avoids using any metadata registry for version management. 


\subsection{ACID transactional semantics}

ACID transaction support is the key feature driving the design of Týr. Transactions provide the user with an expressive way to indicate the relation between a set of queries, and to ensure the correctness of the resulting operation that is either fully applied, either rejected as a complete unit. We argue that co-designing a storage system with its transaction processing provides the following features without requiring additional protocols:

Consistent data replication. Replicating an object for fault-tolerance is a significant challenge for distributed systems not relying on a centralized metadata management service. Indeed, all the replicas must agree on an ordering between the successive writes. A common method for doing so is to rely on logical clocks or on arbitrary ordering based, for example, on the local time the write has been applied on the server receiving the operation from the client. Transactions inherently enable the nodes holding the replicas of any given chunk to agree on a serializable order. From the point of view of the storage system, multiple replicas of a chunk are considered as different objects to which the same operation is applied in the context of a transaction.

Version bookkeeping. A significant challenge in versioned systems is to determine when it is safe to prune old versions of a chunk that are not used anymore. We propose to rely on the transaction algorithm so that all metadata nodes for a blob are able to agree on the older version of the blob to keep, and piggyback that information to the nodes storing chunk replicas of this blob.

Yet, designing an efficient transaction commit protocol in a distributed environment is hard. Traditional approaches used by relational database systems typically rely on centralized transaction managers aimed at coordinating transaction execution in a cluster. However, this approach has three main drawbacks. First, it creates a single point of failure in the system as well as a potential hotspot, hence defeating the distributed metadata managenent benefits for write scalability. Second, the cost of such transaction ordering is to be paid even when transactions are not conflicting with each other, hence hindering the write performance of the cluster. Finally, some form of 
complex, heavyweight static analysis is usually required to ensure that no long-running transaction is prioritized over and hence blocking small ones from committing.

To alleviate these issues, a new generation of distributed storage systems such as Google's Megastore (50), Google's Spanner (50) or Scatter (67) rely on a Paxos protocol (95) to provide such synchronization, assigning objects to different Paxos groups depending on their key. Besides the inner complexity of the Paxos protocol, this scheme incurs significant overhead for operations spanning objects assigned to different groups. Furthermore, as with centralized transaction managers, the cost of such synchronization impacts all transactions, including those for which it would not be required. Techniques exist to reduce this overhead, but also typically rely on some form of complex static analysis, as in Rococo (125) or Lynx (70).

In contrast with those approaches, the Warp transaction chain protocol (61) introduced in the HyperDex key-value store (61) completely decentralizes transaction processing, so that no transaction manager or defined consensus groups are required. It enables transactions to be ordered on-the-fly, delaying the synchronization until a conflict is detected. This significantly reduces the transaction processing costs in cases where applications are specifically designed to prevent such conflicts from happening, as with many legacy HPC applications. Furthermore, this protocol allows for lock-free reads which, combined with the predictable data distribution, ensure a very low latency for read operations in most cases.

Besides their low overhead, transaction chains also ensure the scalability of the system, which is critical for both HPC and BDA applications. Indeed, Warp enables transactions to only involve the servers that are impacted by the transaction (that is, holding chunks that are read-from or written-to in the context of that transaction). This eliminates hot spots in the system, in addition to reducing communication costs.

\subsection{Atomic transform operations}

Transaction based coordination provides the user with a simple way to express interdepency between related storage operations. However, for simple common patterns, their cost can be further reduced. This is notably the case for read-modify-write operations that require two round-trips between the client and the server: one for reading the current value of the data, and a second to optimistically apply the modifications to 
the storage. There are two main issues with this. First, the two round-trips intuitively hinder the operation latency. Second, the transaction would abort should a conflicting transaction commit between the read and write operations, further increasing latency in addition to leaving up to the developer to handle retries when required.

This problem is well known and was studied extensively in the database and shared memory literature (22; 135; 154). Two common alternative to transaction processing for simple read-modify-write use-cases exist:

Conditional primitives such as compare-and-swap (CAS) provide a solid building block for higher-level operations. Yet, while they effectively decouple the read and write operation from the point of view of the storage system, they yield little to no performance gains when compared to transactions. This is because they still require a query to read the current value of the data, and because a failing CAS operation also requires the developer to manually handle failure scenarios. Nevertheless, they proved to be a solid alternative to lock-based synchronization in HPC systems (43).

Atomic transform primitives provide a way for developers to transfer the responsibility of the data modifications to the storage system. This is particularly interesting when the transform operation is simple, such as arithmetic or bitwise operations. With this scheme, the client does not communicate to the storage cluster the new data to be written, but instead the modification to be applied to the current data. This scheme significantly cuts communication costs by enabling such operations to be performed with a single round-trip. Second, being the operation applied atomically to the storage system significantly reduces the probability of aborts due to conflicting operations. Should such abort happen, it transfers the burden of retry to the storage system and consequently eases the task of the application developer.

Despite their benefits for use-cases such as data aggregation, neither these solutions have been adopted in HPC storage systems to date. The main reason for this is that current HPC file systems are heavily constrained by the POSIX I/O API, which does not include such operations. Furthermore, efficient comparison or conditional checks lack in the local storage abstractions that form the foundation of most HPC storage 
systems (43). We advocate that the transactional foundation of the storage system we propose provides an ideal base for these operations to be supported. Indeed, transaction protocols already include such conditional checks. They can be extended to support both CAS and atomic transform primitives without additional overhead, ensuring the consistency of such operations during the commit phase. 


\section{Chapter 7}

\section{Týr protocols}

Designing the protocols enabling Týr users to read and write data is a difficult task. Indeed, the core design principles of data chunking and multi-version concurrency control raise additional challenges compared to other transactional storage systems. A key reason explaining this difficulty lies in the size of the objects being stored. Transactional key-value stores typically only support values of up to a few megabytes, that can be entirely stored on a single node. In transactional file systems such as the Warp Transactional Filesystem, which do not propose direct read access to the data, the scope of transactions is limited to metadata operations in which the objects are small.

A naive approach consists of considering the multiple chunks that compose a single blob as independent objects, and to apply existing transactional protocols to coordinate write operations. Yet, such implementation would cause significant problems for read operations spanning multiple chunks, which would contradict the following two requirements:

Consistent multi-chunk reads: When serving reads spanning multiple chunks, it is important to ensure that the versions of all chunks that are returned together form a consistent version of the blob. This must be guaranteed even while processing concurrent, conflicting write operations. As such, the versions of each chunk cannot be considered independently from one another.

Repeatable reads: This isolation level is particularly desirable for large objects, whose potentially large size prevents them from being read entirely with a single read operation. Essentially, repeatable reads guarantee that, in the context of a 
transaction, all read operations to the same object will return data from the same consistent blob version. This is particularly challenging in presence of conflicting write operations, as the latest version of the blob can change between two read operations. While multi-version concurrency control solves part of the issue by ensuring that older versions are never overwritten, it does not bring a solution to all problems. In particular, the storage system should ensure that an older chunk version is only deleted when it is safe to do so, i.e. when no running transaction could potentially require that version of the chunk.

We solve these challenges by carefully designing the read and write protocols of Týr, which ensure that these two requirements are met.

\subsection{Lightweight transaction protocol}

Write coordination in Týr leverages the Warp optimistic transaction protocol, whose correctness and performance has been proven in (61). This section provides an overview of Warp, upon which Týr is built.

In order to commit a transaction, the client constructs a chain of servers which will be affected by it. These nodes are all the ones storing the written data chunks, and one node holding the data for each chunk read during the transaction (if any). This set of servers is sorted in a predictable order, such as a bitwise ordering on the IP / port pair. The ordering ensures that conflicting transactions pass through their shared set of servers in the exact same order.

The client addresses the request to the first node of the chain, designated as the coordinator for that request. This node will validate the chain and ensure that it is up-to-date according to the latest ring status. If not, that node will construct a new chain and forward the request to the coordinator of the new chain.

Commit protocol. A linear transactions commit protocol guarantees that all transactions are either successful and serializable, or abort with no effect. This protocol consists of one forward pass to optimistically validate the values read by the client and ensure that they remained unchanged by concurrent transactions, followed by a backward pass to propagate the result of the transaction - either success or failure and actually commit the changes to memory. Dependency information is embedded by 


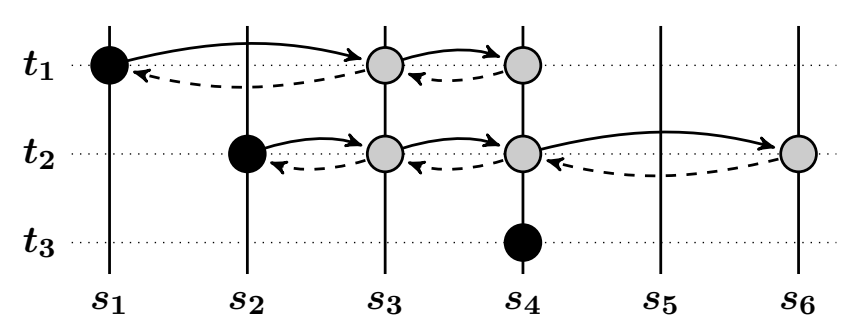

(a) Propagation graph

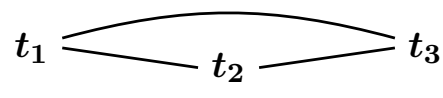

(b) Dependency graph
Figure 7.1: Warp lightweight transaction protocol overview. In this example, the directionality of the edge $\left(t_{1}, t_{2}\right)$ will be decided by $s_{4}$, last common server in the transaction chains, during the backwards pass. Similarly, the directionality of $\left(t_{2}, t_{3}\right)$ will be decided by $s_{4}$.

the nodes in the chain during both forward and backward passes to enforce a serializable order across all transactions. A background garbage collection process limits this number of dependencies by removing those that have completed both passes.

Transaction validation. The coordinator node does not necessarily own a copy of all the chunks being read by every transaction, which are distributed across the cluster. As such, one node responsible for a chunk being read in any given transaction must validate it by ensuring that this transaction does not conflict nor invalidates previously validated transactions, for which the backward pass is not complete. Every node in the commit chain ensures that the transactions do not read values written by, or write values read by previously validated transactions. Nodes also check each value against the latest one stored in their local memory to verify that the data was not changed by a previously committed transaction. The validation step fails if transactions fail either of these tests. A transaction is aborted by sending an abort message backwards through the chain members that previously validated the transaction. These members remove the transaction from their local state, thus enabling other transactions to validate instead. Servers validate each transaction exactly once, during the forward pass through the chain. As soon as the forward pass is completed, the transaction may commit on all servers. The last server of the chain commits the transaction immediately after validating it, and sends the commit message backwards to the chain.

Transaction serialization. Enforcing a serializable order across all transactions requires that the transaction commit order does not create any dependency cycles. To this end, a local dependency graph across transactions is maintained at each node, with 
the vertices being transactions and each directed edge specifying a conflicting pair of transactions. A conflicting pair is a pair of transactions where one transaction writes at least one data chunk read or written by the other. Whenever a transaction validates or commits after another one at a node, this information is added to the transaction message sent through the chain: the second transaction will be recorded as a dependency of the first. This determines the directionality of the edges in the dependency graph. A transaction is only persisted in memory after all of its dependencies have committed, and is delayed at the node until this condition is met.

Figure 7.1 illustrates this protocol with an example set of conflicting transaction chains and the associated dependency graph. This example shows three transaction chains executing. Figure 7.1a shows the individual chains with the server on which they execute. The black dot represents the coordinating server for each transaction, the plain lines the forward pass, and the dashed lines the backwards pass. Because they overlap at some servers, they form conflicting pairs as shown on the dependency graph in Figure $7.1 \mathrm{~b}$. The directionality of the edges will be decided by the protocol, as the chain is executed.

\subsection{Handling reads: direct, multi-chunk and transactional protocols}

As per our requirements, the read protocol of Týr needs to ensure the correctness of consistent reads spanning multiple chunks while providing repeated reads isolation in the context of the transaction or direct reads whenever possible. Three cases need to be considered:

Direct reads. This protocol is used outside a transaction, when the read operation can be served from a single chunk.

Multi-chunk reads. This protocol is used outside of a transaction, when the read operation spans multiple chunks. It ensures that the client is returned a consistent view of the blob.

Transactional, repeatable reads. This protocol ensures that, inside of a transaction, the client is able to issue successive read operations on the same blob, 
potentially at different offsets. Each of these individual read operations must be performed on the same blob version that was accessed by the first read operation.

\subsubsection{Direct reads}

Direct reads is a desirable feature that stays at the core of the performance of keyvalue stores. It enables the client to address a read request directly to a node of the storage system that holds this information, without any prior communication with any metadata server.

The client leverages the information it holds about the distribution of the data in the cluster to first locate a node holding the desired data, by hashing its key and chunk number (calculated from the data offset). The read request is sent directly to that node. The node responds with the data from the latest committed version of the chunk as per its internal dependency graph.

Should the request have been sent by the client to a wrong node in the first place, the node receiving the request uses its own knowledge of the data distribution to forward the request to the correct node. This node returns the requested data directly to the client. Such errors may happen if the token ring has changed since the last time the client updated this information. To avoid further errors, the new state of the token ring is piggybacked to the response message so that the client can update it without requiring further requests.

This protocol is the most efficient Týr implements, only involving a single node of the storage system. However, it is not applicable to multi-chunk reads as none of the servers involved do have information about the set of chunk versions that form a consistent view of the blob. For the same reason, inside a transaction, it cannot offer repeatable reads. As such, the Týr client only uses this protocol for reads spanning exactly one chunk performed outside the context of a transaction.

\subsubsection{Multi-chunk reads}

When reading a portion of a blob which overlaps multiple chunks, it is necessary to obtain the version identifiers of each of those chunks forming a consistent version of the blob, as previously explained in Section 6.3. Only the version manager nodes for the blob to be read hold complete information about the chunk versions that form 


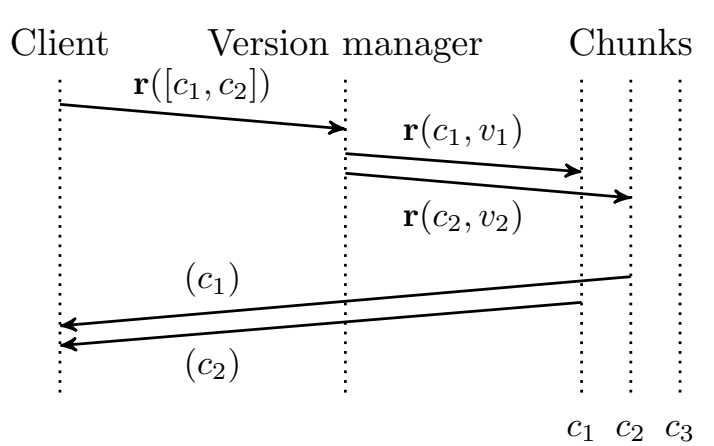

Figure 7.2: Týr read
protocol for a read span-
ning multiple chunks. The
client sends a read query
for chunks $c_{1}$ and $c_{2}$ to the
version manager, which re-
lays the query to the servers
holding the chunks with the
correct version information.

a consistent blob version. As such, the protocol used for served multi-chunk reads leverages these nodes to determine which versions of each accessed chunk to read from.

To perform a multi-chunk read, the client directs its request to any of the version manager nodes for the blob. Similarily to the direct read protocol, these nodes are determined using the client knowledge about the token ring, the version managers being the same servers holding data for the first chunk of the blob.

From the version information it holds about the blob, the version manager determines, for the most recent committed version of the blob, the versions of each of the chunks accessed by the read operation. It also uses its own knowledge of the token ring to locate the servers holding the data for these chunks, selecting one at random. The read request is forwarded in parallel to each of these servers, including in the request the exact version identifier to read from. Each server replies directly to the client with the requested data. The client reconstructs the data in memory as it receives responses for each chunk. The protocol is illustrated by Figure 7.2 ,

By instructing servers holding chunk data to provide a specific version of each chunk, this protocol ensures that the client is returned a consistent version of the blob. Yet, it does not allow for repeatable reads. As such, the client selects this protocol for multi-chunk reads that are executed outside of the context of a transaction.

\subsubsection{Transactional, repeatable reads}

In the context of a transaction, a further modification of the multi-chunk read protocol is required to provide repeatable reads. The transactional read algorithm is essentially a combination of the multi-chunk and direct read protocols, combining the correctness 


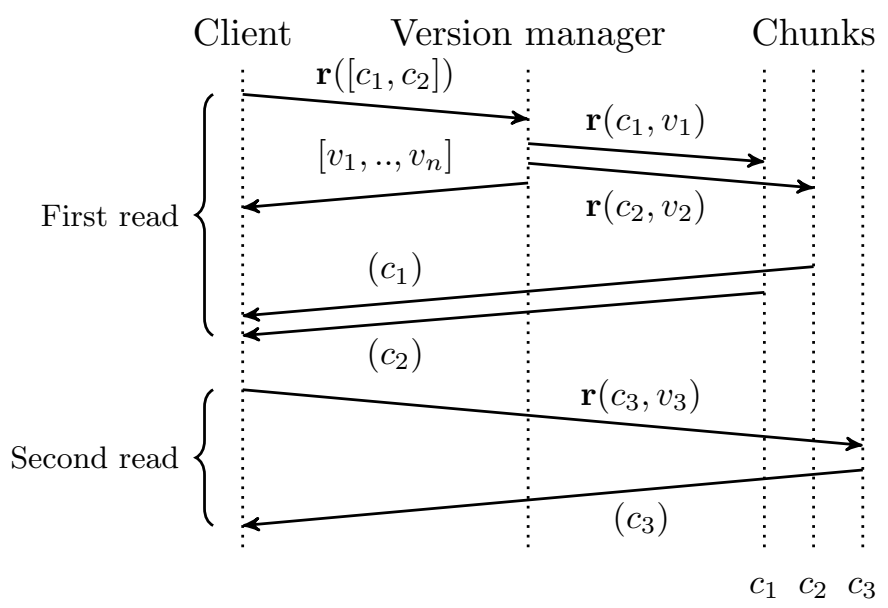

Figure 7.3: Týr read protocol inside a transaction. The client sends a read query for chunks $c_{1}$ and $c_{2}$ to the version manager, which relays the query to the servers holding the chunks with the correct version information, and responds to the client with a snapshot of the latest chunk versions. Subsequent read on $c_{3}$ is addressed directly to the server holding the chunk data using the aforementioned chunk version information.

of the former for the first read request with the simplicity and velocity of the former for subsequent requests.

The first read request is processed as for the multi-chunk read protocol: the request is transmitted to a version manager which in turn forwards the request with the appropriate version identifiers to the servers holding the requested data. A key difference enabling for repeatable reads is that the version manager node returns to the client the whole set of version identifiers for each chunk that together form the last committed blob version. This version information is cached by the client in the context of the transaction.

Upon executing subsequent read requests for the same blob, the client extracts from that cached version information the version identifiers for each chunk to be accessed. The data is requested directly from the servers holding the chunk data as in the direct read protocol. It includes in the request message the version identifier to read from. The whole process is illustrated by Figure 12.1 .

All this version management is done internally by the client, and is kept hidden from the application. This protocol enables the client to read the same consistent version of the blob even in presence of concurrent writes to the chunks being accessed. 


\subsection{Handling writes: transactional protocol, atomic trans- forms}

The complexity of version management is taken care of by the read protocol. As such, the basic write protocol of Týr is largely based on the unmodified Warp protocol. We introduce two modifications that are necessary for keeping the metadata managers up-to-date, and supporting in-place, atomic transform operations.

\subsubsection{General protocol}

The version managers of a blob have complete information about the successive versions of the blob. Thus, the version managers of any given blob have to be made aware of any write to this blob. We achieve this by systematically including the version managers in the commit chain, in addition to the nodes holding the chunk data. The version managers of all the blobs being written to in a given transaction are ordered using the same algorithm used for the rest of the chain. This ensures that, during the backward pass of the transaction commit protocol, the transaction will have successfully committed on every chunk storage node before it is marked as committed on the version manager nodes.

Version managers are inserted at the beginning of the chain; they are as such the first nodes informed of a transaction during the forward pass and of a successfully committed transaction during the backwards pass. The latter fact is key to the correctness of both the multi-chunk and transactional read protocols. This ordering also ensures that these

nodes are last informed of successful transactions during the backwards pass. This ensures that a version manager always returns versions that have also been marked committed by the nodes holding the chunk data.

Correctness: A transaction $t_{1}$ conflicts with another transaction $t_{2}$ only if it reads a chunk written to by $t_{2}$, if it writes to a chunk being read to by $t_{2}$, or if it writes to a common set of chunks. The chain commit protocol enables the first two cases to be detected during the forward pass, regardless of the chain ordering. Placing the ordered list of version managers at the beginning of the chain does not break the correctness of the chain commit algorithm: if $t_{1}$ and $t_{2}$ write to a common set of blob chunks, the version managers for these blobs will be included in both commit chains, sorted in the 
same order, and will be inserted at the beginning of each. Consequently, two conflicting transactions will keep the same chain relative order, as required by the commit protocol.

\subsubsection{Atomic transforms}

Atomic transforms provide a convenient way to express simple binary operations, such as arithmetic or bitwise operations. They operate on a binary value of fixed size located at a specific offset of the blob. The size of the binary value depends on the operation applied, but typically does not exceed 64 bits.

We modify the write protocol to support atomic operations. Two cases are possible: The binary value to be modified is contained in a single chunk. Handling of this case leverages the consistent ordering natively provided by Týr transaction protocol. During the forward pass, an atomic update operation is handled as any write operation. During the backwards pass, all servers holding a replica of the binary value to be modified atomically apply the atomic operation. As per multi-version concurrency control, the operation is applied on a new version of the chunk.

The binary value to be modified spans multiple chunks. Applying the operation in this context provides additional challenge, as the value to be modified is not fully contained on a single machine. When such case is detected, Týr internally transforms the operation into a two-step read-update-write operation that is performed by the storage server on behalf of the user. In the context of the user transaction, the transaction coordinator issues a read request for the binary value to be modified, and inserts in the transaction a write operation with the updated value. Contrary to the general case, this succession of a read and write operation can cause the transaction to fail in situations of concurrent, conflicting write accesses. Should this happen, the operation is automatically and transparently retried by the transaction coordinator.

Atomic operations for binary values spanning multiple chunks incur a significant write overhead because of the required two-step operation and of the possible retries. Yet, this case should be rare in practice. This is because of the relatively high size of a data chunk (64 MB by default) compared to that of the binary value (up to 64 bits). The application developer can eliminate this overhead entirely by ensuring that atomic operations are always aligned to 64 bits. 


\subsection{Bookkeeping: purging expired versions}

Týr uses multiversion concurrency control as part of its base architecture in order to handle lock-free read / write concurrency. Týr also uses versioning in support of the read protocol, specifically to achieve write isolation and ensure that a consistent version of any blob can be read even in the presence of concurrent writes. A background process called version garbage collector is responsible for continuously removing unused chunk versions on every node of the cluster. A chunk version is defined as unused if it is not part of the latest blob version, and if no version of the blob it belongs to is currently being read as part of a transaction.

How to determine the unused chunk versions? The transaction protocol defines a serializable order between transactions. It is then trivial for every node to know which is the last version of any given chunk it holds, by keeping ordering information between versions. Determining whether a chunk version is part of a blob version being read inside a transaction is however not trivial. Intuitively, one way to address this challenge is to make the version managers of the blob responsible for ordering chunk version deletion. This is possible because the read protocol ensures that a read operation on any given node inside a transaction will always hit a version manager of this blob. Hence, at least one version manager node is aware of any running transaction performing a read operation in the cluster. Finally, the version managers are aware of the termination of a transaction as they are part of the commit chain.

\subsubsection{Detecting and deleting unused chunk versions}

The key information allowing to decide which chunk versions to delete is stored by the version managers. A node will never delete any chunk version unless it has been cleared to do so by every version manager of the blob the chunk belongs to. Version managers keep a list of every running transaction for which they served as relay for the first read operation on a blob. Upon receiving a read operation from a client, a version manager increases a usage counter on the latest version of the blob (i.e. the blob version used to construct the chunk version list as per the transactional read protocol detailed in Section 7.2.2.1). This usage counter is decremented after the transaction is committed, or after a defined read timeout for which the default is 5 minutes. Such timeout is necessary in order not to maintain blob versions indefinitely in case of a 
client failure. The list of currently used chunk versions of any blob is communicated to all the nodes holding the chunks for this blob by means of the transactional commit protocol: for any given transaction, for every blob being written to by the transaction, each version manager piggybacks to the forward and backward pass messages the list of chunk versions currently in use. This guarantees that every node which is part of the chain will get this chunk version usage information as part of the protocol, either during the forward or the backward pass.

For any given blob, the version garbage collector of any node is free to delete any chunk version that $(a)$ is not the latest, $(b)$ is older than the latest transaction for which version usage information for that blob has been received from the version managers, and $(c)$ was not part of the chunk versions in use as part of the latest version usage information received for this blob. The version garbage collector may also safely delete any chunk version older than the read timeout which has not been superseded by a newer version.

\subsubsection{Optimizing the message size}

Týr limits the commit message overhead when the number of chunk versions currently in use is high. Specifically, when this number reaches a set threshold, the currently used chunk versions are piggybacked by the version managers to the transaction messages as a Bloom filter (28). A Bloom filter is a space-efficient probabilistic data structure that is used to test whether an element is member of a set. The version garbage collector can efficiently check whether a blob version is in the set of currently running transactions. Bloom filters are guaranteed never to cause false negatives, ensuring that no currently used chunk will be deleted. However, bloom filters can return false positives, which may cause a chunk version to be incorrectly considered as being used. These chunk versions will be eventually deleted during a subsequent transaction involving this blob, or once the read timeout was exceeded. The false positive probability of a bloom filter depends on its size, and is a system parameter. The default is a $0.1 \%$ error probability. With this configuration, 100 concurrent running transactions on each 3 version managers of a blob would cause a commit message overhead of less than 0.6 Kilobytes. 


\section{Chapter 8}

\section{Implementation details}

We implement the design principles of Týr in a software prototype. This includes the Týr server, an asynchronous $\mathrm{C}$ client library, as well as partial C++ and Java bindings. The server itself is approximately 25,000 lines of Rust and GNU C code. This chapter describes key aspects of the implementation.

Týr is designed following a distributed, modular and loosely coupled architecture. Each node runs a set of different modules, executed on different threads. Figure 8.1 shows the interaction between these modules:

- The Cluster Manager maintains the ring state between cluster nodes using a weakly consistent gossip protocol (52) to propagate information around the cluster (i.e. ring position allocations) and a $\phi$ accrual failure detector (78) to detect and confirm node failures.

- The Query Processor coordinates the requests and handles the transaction protocol using cluster information from the cluster manager $(5,6)$. It acts as the interface to the storage agent $(7,8)$.

- The Query Router is the main communication interface between a server and both the rest of cluster and the clients. It receives incoming client requests(1), parses, validates and, if necessary, forwards them to the appropriate server according to cluster state information (2, 3). It then forwards them to the query processor $(4,9)$, and responds to the client (10).

- The Storage Agent is responsible for the persistent storage and retrieval of both data and version information. 


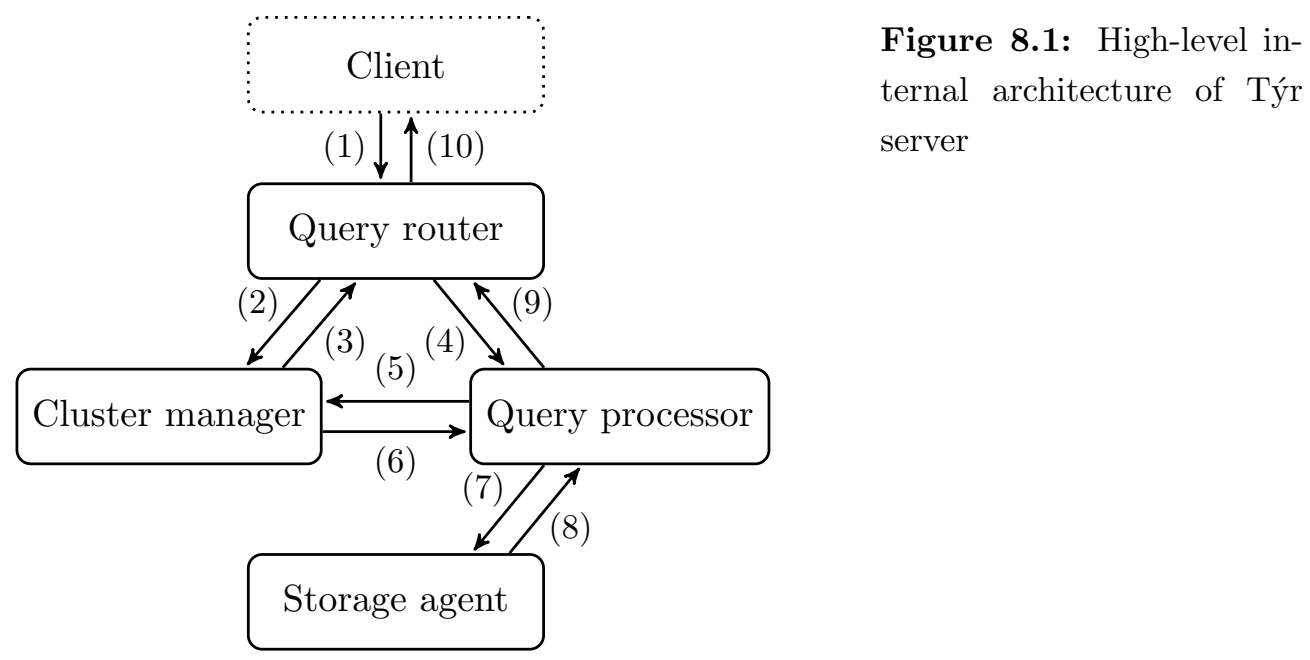

\subsection{Multiple, configurable keyspaces}

The notion of keyspace is central to the design of Týr. A keyspace offers an isolated namespace which contains related sets of objects. We design Týr so that multiple keyspaces may co-exist in parallel in the same cluster. Different keyspaces would typically be used to logically separate resources belonging to different users or applications. Keyspaces in Týr are akin to buckets in Amazon S3. They offer the following features: Access control: Access control in Týr is implemented at the keyspace level. These permissions are designed to control read and / or write access to a keyspace for different groups and users. Indeed, because of the requirements of the direct read feature, implementing access control at the object level would be a costly operation, which we argue is little used in practice in both HPC and BDA systems. Access is provided in an all or nothing fashion: a permission on a keyspace is granted for all objects in that keyspace.

Specific configuration: Several Týr configuration parameters can be set on a perkeyspace fashion. This enables to fine-tune the behavior of a bucket depending of precise application requirements. The parameters tunable for each keyspace notably include the chunk size, replication factor and whether or not to enable multi-version concurrency control. For example, a keyspace used for cache, in which data safety is not critical, would typically reduce the replication factor in order to increase read performance. In a keyspace used as a key-value store, where keys are completely replaced 
at each write, multi-version concurrency control would also be disabled to reduce the small performance and storage overhead associated with this feature.

In the current implementation, all keyspaces are distributed across all nodes in the cluster. The configuration associated with each keyspace is stored by each node. This configuration is statically set when the keyspace is created, and cannot be altered. All keyspaces use distinct consistent hashing parameters, chosen at random when the keyspace is created, so that the mapping of blob keys to specific nodes is different for each keyspace.

\subsection{Persistent data storage}

Data persistence in Týr is largely based on the RocksDB key-value storage system. It is especially suited for storing data on flash drives. It has a Log-Structured-MergeDatabase (LSM) design with flexible tradeoffs between Write-Amplification-Factor, Read-Amplification-Factor and Space-Amplification-Factor. These guarantee that the amount of I/O performed read or write operation is small, and that the total disk space used is close to that of the data it contains. It features important characteristics such as multi-threaded compactions, which make it specially suitable for storing multiple terabytes of data in a single database.

Each keyspace in Týr is mapped on each node to a different RocksDB database. This greatly eases the design of the keyspace drop operations, which can be performed by dropping the database as a whole on all nodes in the cluster. This design could also permit future releases to distribute data over different storage tiers for each keyspace to accomodate for diverse performance / storage cost trade-offs in the same cluster.

\subsection{Fault-tolerance}

Fault-tolerance is critical to the design of Týr. The storage system is able to tolerate the loss or malfunction of nodes in the cluster gracefully. Its implementation heavily relies on a Gossip protocol to detect failures, as well as failure-awareness of the protocols and mechanisms used to handle read and write requests. 


\subsubsection{Gossip-based failure detection}

Týr relies on an efficient Gossip protocol to solve three major challenges:

Cluster membership Týr maintains cluster membership lists at each node, so that each node in the cluster has a complete view of all the nodes that compose it. This information is critical for the data distribution with consistent hashing.

Failure detection and recovery Týr automatically detects failed nodes, and notifies the rest of the cluster of any failure. This information is used internally to re-execute transactions impacted during their execution by failed nodes. Týr will attempt to recover failed nodes by reconnecting to them periodically.

Configuration and metrics propagation Týr leverages Gossip to exchange and synchronize configuration loosely across the cluster. For example, creating or deleting a keyspace are operations propagated across the cluster using Gossip.

Gossip is based on the SWIM protocol (52). It is performed over UDP with a configurable but fixed fanout and interval. This ensures that network usage is constant with regards to number of nodes. Complete state exchanges with a random node are done periodically over TCP, but less often than gossip messages. This increases the likelihood that the membership list converges properly since the full state is exchanged and merged.

Failure detection is done by periodic random probing using a configurable interval. If the node fails to acknowledge within a reasonable time (typically some multiple of the average round-trip time), then an indirect probe is attempted. An indirect probe asks a configurable number of random nodes to probe the same node, in case there are network issues causing our own node to fail the probe. If both our probe and the indirect probes fail within a reasonable time, then the node is marked "suspicious" and this knowledge is gossiped to the cluster. A suspicious node is still considered a member of cluster. If the suspect member of the cluster does not dispute the suspicion within a configurable period of time, the node is finally considered dead, and this state is then gossiped to the cluster. 


\subsubsection{Handling storage node failures}

Týr gracefully handles server node failures using two mechanisms. Data replication guarantees the availability of the data in case of node failures. Transaction re-execution mechanisms detect the transactions impacted by failing nodes, automatically retrying them should such an event occur.

\subsubsection{Data replication}

The data in Týr is replicated on a number of nodes that is configurable per keyspace. This reduces the likelihood of data loss should nodes fail or be temporarily available in the cluster. Data availability is guaranteed as long as the number of failing nodes is under the configured replication factor for a given keyspace.

While the availability is guaranteed, node failures can severely impact the performance of the cluster as well as the request latency. Indeed, failing nodes will concentrate the requests for ranges of keys to a smaller number of servers. In its current state, Týr does not automatically rebalance the data when nodes are failing.

Týr clients automatically retry timed-out read and write requests using a different data replica as source should a defined timeout elapse before a response is received.

\subsubsection{Transaction re-execution}

When a node fails, all executing transactions that do include that node in the chain are severely affected. Indeed, this can prevent a transaction from executing correctly. Each node in the chain being responsible for forwarding the message to the next member in the chain, the basic protocol does not tolerate failing members.

This problem is solved using a transaction re-execution mechanism. The transaction coordinator constantly monitors failing nodes in the cluster. Should a node fail that is included in any chain the node is coordinator for, it will calculate a new transaction chain that excludes the failing node. The transaction protocol is re-started with that new chain. Each node in the chain checks this re-execution against their local state in order to verify whether they have processed this transaction already. This ensures that the transaction will be resumed from its point of failure.

In its current state, Týr does not support simultaneous failures of the node coordinator and of another node in any given transaction chain. 


\subsection{Event-driven server design}

The internal design of Týr largely follows best practices. It seeks to use the full capabilities of the machine it runs on by maximizing parallelism and reducing as much as possible the coordination between execution threads. To do so, Týr uses a fully non-blocking, event-driven design based on the LibUV library (85). This principle is used at all stages of Týr: for network I/O, for internal query processing as well as for persistent storage access.

\subsubsection{Client-server and internal cluster communication}

The efficiency of the client-server communication is key to the overall performance of the storage server. Two libraries are used for guaranteeing the performance of Týr for that aspect:

Data serialization Týr uses a custom data serialization, in which each message is the concatenation of the serialized metadata and, if the operation is a write operation, of the compressed data associated with it. The metadata is serialized using Google's FlatBuffers library (8), which does not require data parsing, unpacking or memory allocation for decoding the metadata. It is among the most efficient serialization libraries available. The algorithm used for compressing the data is LZ4 (46), which is able to process data at near-memory speed by leveraging various low-level hardware optimizations.

Low-level wire protocol The low-level transmission of requests, both client-server and internal to the cluster, is based on the KCP protocol (10). KCP provides fast, ordered and error-checked delivery of streams over UDP packets, guaranteeing lower latency and overall higher throughput than TCP. It is notably able to adapt its configuration to the quality of the network between two nodes, hence improving usage of the available bandwidth. Being based on UDP, this protocol is ideal for Týr as it does not require handshakes before transmitting data. This notably supports the read protocols, in which the nodes responding to a request can be different from the one the request was initially sent to. 


\subsubsection{Processing queues}

Týr includes multiple parallel, non-blocking processing queues for highly-efficient concurrent query execution. When a request arrives, it is immediately pushed to one of the processing queues using a round-robin algorithm. The processing queue, or query processor, is then responsible for acting upon the request. Specifically, it coordinates all actions that should be taken in response to that request: retrieving information from the storage agent, forwarding the request to another node or processing a transaction.

I/O operations in Týr are non-blocking. Callbacks are used at each stage of the processing of a request for asynchronous operations. The number of concurrently executing operations in a single queue is bounded in order to guarantee a low processing latency even at higher loads.

A transaction arbiter constitutes the only resource shared between processing queues. It essentially maintains the transaction dependency graph, and is leveraged to make decisions on the ordering of conflicting transactions. 


\section{Part III}

\section{Evaluation: Týr in support of HPC and BDA convergence}





\section{Chapter 9}

\section{Real-time, transactional data aggregation in support of system monitoring for BDA}

In this chapter, we seek to prove the relevance of transactional blobs as a storage model for purpose-built application. In particular, we demonstrate the performance benefits of the two key features of Týr: transactions and atomic operations.

We base these experiments on a real-world, BDA application that is part of ALICE (A Large Ion Collider Experiment) (14) at CERN (European Organization for Nuclear Research) (100). In particular, we consider the needs of the monitoring system collecting and aggregating in real-time telemetry events from more than 80 datacenters around the world: MonALISA (101; 102).

We use Týr to process and aggregate real data from MonALISA, and compare its performance with other state-of-the-art systems on the Microsoft Azure cloud platform. We prove that Týr throughput outperforms its competitors by up to $75 \%$ when faced with transactional operations while providing significantly higher consistency guarantees. We scale the storage system to up to 256 nodes to demonstrate the scalability of such transactional operations. 


\subsection{ALICE and MonALISA}

ALICE (14) is one of the four LHC (Large Hadron Collider) experiments running at CERN (100). ALICE collects data at a rate of up to 4 Petabytes per run and produces more than $10^{9}$ data files per year. Tens of thousands of CPUs are required to process and analyze them. The CPU and storage capacities are distributed over more than 80 datacenters around the world.

We focus on the management of the monitoring information collected in real-time about all ALICE resources. More than 350 MonALISA services are running at sites around the world, collecting information about ALICE computing facilities, local- and wide-area network traffic, and the state and progress of the many thousands of concurrently running jobs. This yields more than 1.1 million measurements pushed to MonALISA, each with an update frequency of one second. In order to be presented to the end-user, the raw data is aggregated to produce about 35,000 system-overview metrics, and grouped under different time granularity levels.

\subsubsection{Managing monitoring data: what could be improved}

The current implementation of ALICE is based on a PostgreSQL database (122). Aggregation is performed by a background worker task at regular intervals. With the constant increase in volume of the collected metrics, this storage architecture becomes inefficient. Time-series databases such as OpenTSDB (146) or KairosDB (126) were considered to replace the current architecture. However, storing each event individually, along with the related metadata such as tags, leads to a significant overhead. In the context of MonALISA, the queries are known at the time measurements and stored by the system. This opens the way to a highly-customized storage backend using a data layout that would at the same time dramatically increase throughput, reduce metadata overhead, and ultimately lower both the computing and storage requirements for the cluster. We use Týr to develop such a backend.

\subsubsection{The need for transactions}

The blob-based storage layout for the MonALISA system is as follows. All measurements (timestamp, measurement) are appended to a per-generator blob. Measurements are then averaged over a one-minute window with different granularity levels 


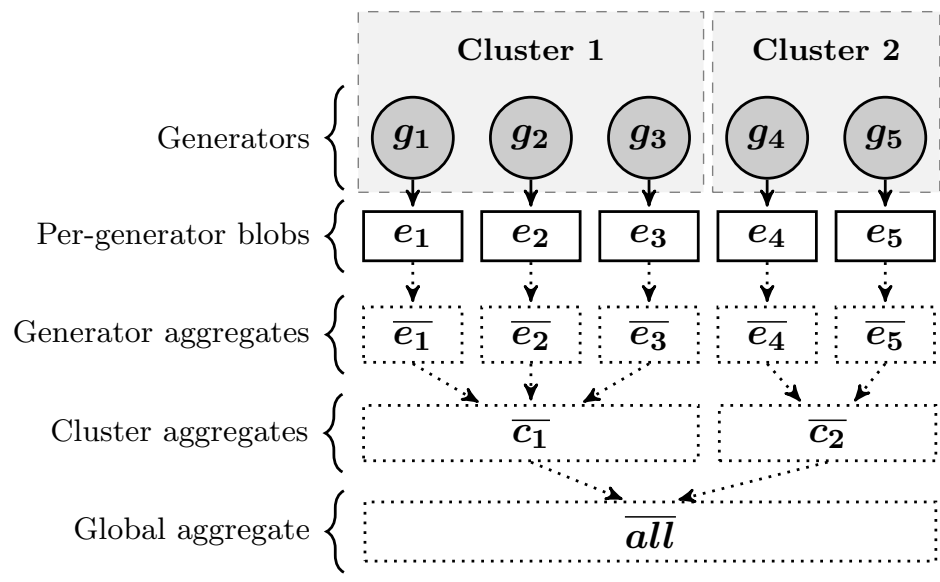

Figure 9.1: $\quad$ Simplified MonALISA data storage layout, showing five generators on two different clusters, and three levels of aggregation. Solid arrows indicate events written, dotted arrows represent event aggregation. Each rectangle indicates a different blob. Dotted rectangles denotes aggregate blobs.

(machine, cluster, site, region, and job). This layout is explained in Figure 9.1. Updating an aggregate is a three-step operation: read old value, update it with the new data, and write the new value (read-update-write). In order to guarantee the correctness of such operations, all writes must be atomic. This atomicity also enables hot snapshotting of the data. As an optimization for aggregate computation, it is desirable for the read-update-write operations to be performed in-place, using Týr's atomic transform operations.

\subsection{Experimental setup}

We deploy Týr on the Microsoft Azure Compute (49) platform on up to 256 nodes. For all experiments, we used D2 v2 general-purpose instances, located in the East US region (Virginia). Each virtual machine has access to 2 CPU cores, 7 GB RAM and 60 GB SSD storage. The host server is based on $2.4 \mathrm{GHz}$ Intel Xeon E5-2673 v3 processors and is equipped with 10 Gigabit Ethernet connectivity.

We compare Týr with Rados (169) and BlobSeer (127). We also plot the results obtained with Microsoft Azure Storage Blobs (39), a managed blob storage system available on the Microsoft Azure platform. It comes in three flavors: append blobs, block blobs and page blobs. Append blobs are optimized for append operations, block blobs are optimized for large uploads, and page blobs are optimized for random reads and writes. These experiments use Rados 0.94.4 (Hammer), BlobSeer 1.2.1, and the version of Azure Storage Blobs available at the time of these experiments (April 2016). 
Using a real-world data set: We use a dump of real data obtained from MonALISA. This data set is composed of $\sim 4.5$ million individual measurement events, each one being associated to a specific monitored site. We used multiple clients to replay these events, each holding a different portion of the data. Clients loop over the data set to generate more events when the size of the data is not sufficient. The data is stored in each system following the layout described in Section 9.1.2. The read operations are performed by querying ranges of data, simulating a realistic usage of the MonALISA interface. In order to further increase read concurrency, the data is queried following a power-law distribution.

Ensuring write consistency on non-transactional systems: Because of the lack of native transaction support in Týr competitors, we use ZooKeeper 3.4.8 (80) (ZK), an industry-standard, high-performance distributed synchronization service, which is part of the Hadoop (145) stack. Zookeeper allows us to synchronize writes to the data stores with a set of distributed locks. ZooKeeper locks are handled at the lowestpossible granularity: one lock is used for each aggregate offset (8-byte granularity), except for Azure in which we have to use coarse-grained locks (512-byte granularity). Indeed, Azure page blobs, which we use for storing the aggregates because of their random write capability, require writes to be aligned on a non-configurable 512-byte page size. Furthermore, append blobs are not suited for operating on small data objects such as MonALISA events, as they are limited to 50.000 appends.

Throughout our experiments, we measure the relative impact of the choice of ZooKeeper as a distributed lock provider. Overall, the results show that ZooKeeper accounts for less than $5 \%$ of the total request latency for write storage operations. Although faster, more optimized distributed locks such as Redis (42) may be available, this would not significantly impact the results.

\subsection{Transactional write performance}

High transactional write performance is the key requirement that guided the design of Týr. In this section we provide its baseline performance under a transactional workload. We measure the transactional write performance of Týr, Rados, BlobSeer and Microsoft Azure Blobs with the MonALISA workload. 


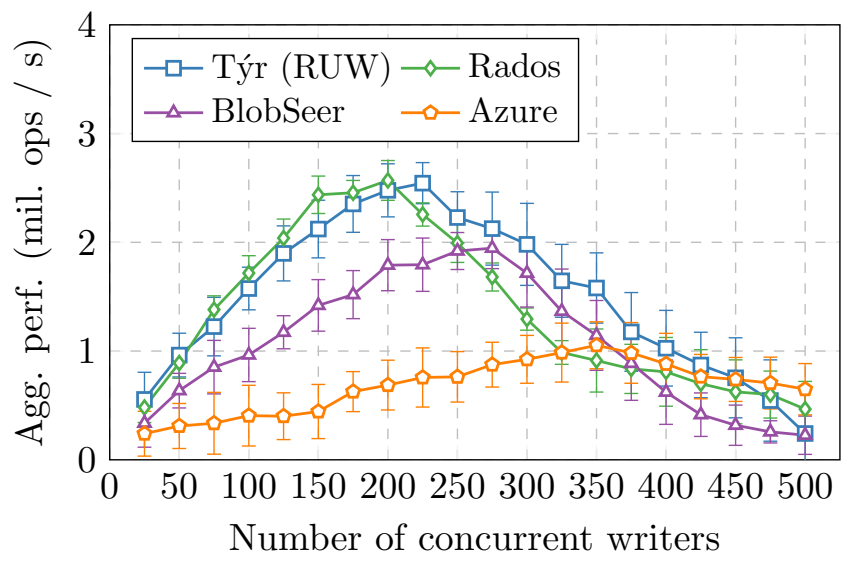

Figure 9.2: Baseline performance of Týr, Rados, BlobSeer and Azure Storage, varying the number of clients, with $95 \%$ confidence intervals.

We perform data aggregation in Týr using atomic transforms as well as with transactional read-update-write operations. This is to provide a fair baseline for comparison with other systems. Indeed, Týr is the only system to support such semantics. Týr transactions are used to synchronize the storage of the events and their indexing in the context of a concurrent setup. All systems are deployed on a 32-node cluster, except for Azure Storage which does not offer the possibility to tune the number of machines in the cluster.

\subsubsection{Comparing with non-transactional systems}

First, we want to better understand the true cost of the lightweight transaction algorithm Týr implements. To do so, we run the MonALISA workload using transactions on Týr, using only read-update-write aggregation. We do not use any form of synchronization on the other systems, as none do support such semantics natively. In this setup, Týr is the only system guaranteeing the correctness of the operations.

We plot the results in Figure 9.2. Overall, Týr performance is similar to the one Rados, while still providing significantly stronger consistency guarantees. These results are explained by its lock-free, multi-version concurrency control design which supports its performance in such highly-concurrent workloads. The performance gap with BlobSeer is mainly due to the lower overhead of metadata management in Týr compared to the distributed tree BlobSeer relies on. 


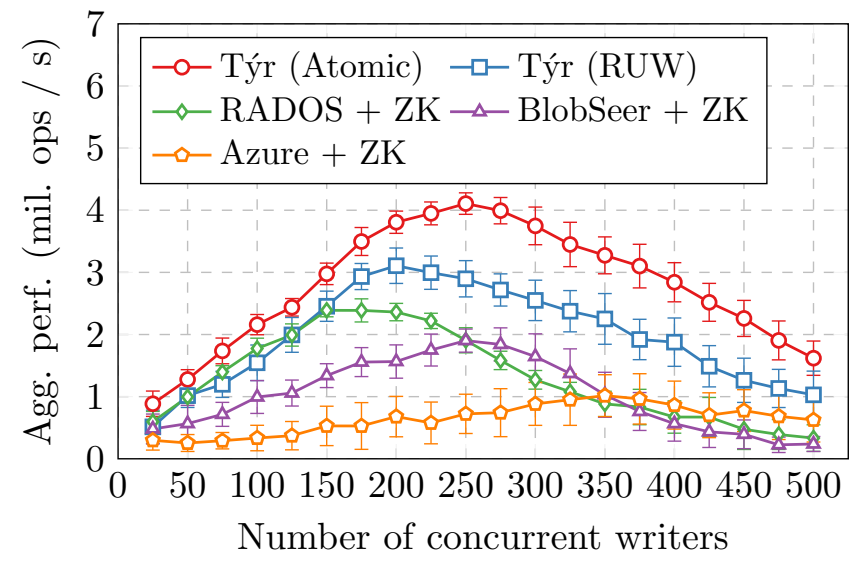

Figure 9.3: Synchronized write performance of Týr, Rados, BlobSeer and Azure Storage, varying the number of clients, with $95 \%$ confidence intervals.

\subsubsection{Comparing with lock-based transactions}

In this section we provide a comparison while ensuring data consistencty for all systems. We use ZooKeeper to synchronize writes on Rados, BlobSeer and Azure Blobs.

The results, depicted in Figure 9.3, show that the Týr peak throughput outperforms its competitors by $78 \%$ while supporting higher concurrency levels. Atomic updates allowed Týr to further increase performance by saving the cost of read operations for simple updates. The significant drop of performance in the case of Rados, Azure Storage and BlobSeer at higher concurrency levels is due to the increasing lock contention. This issue appears most frequently on the global aggregate blob, which is written to for each event indexed. In contrast, our measurements show that Týr's performance drop is due to CPU exhaustion, mainly because of the additional resources required for handling incoming network requests. Under lower concurrency, however, we can see that the transaction protocol incurs a slight processing overhead, resulting in a comparable performance for Týr and Rados when the update concurrency is low. BlobSeer is penalized by its tree-based metadata management which incurs a non-negligible overhead compared to Týr and Rados. Overall, Azure shows a lower performance and higher variability than all systems. At higher concurrency levels however, Azure performs better than both Rados and BlobSeer. This could be explained by a higher number of nodes in the Azure Storage cluster, although the lack of visibility into its internals doesn't allow us to draw any conclusive explanation. We can observe the added value of atomic transform operations in the context of this experiment, which enables Týr to increase its performance by $33 \%$ by cutting the cost of read operations for simple 


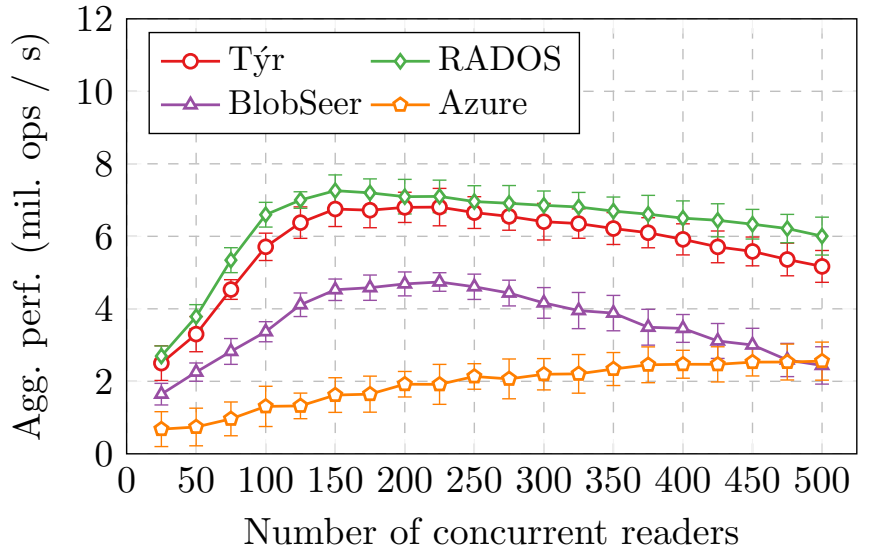

aggregate updates and reducing the transaction failure rate.
Figure 9.4: Read performance of Týr, Rados, BlobSeer and Azure Storage, varying the number of clients, with $95 \%$ confidence intervals.

\subsection{Read performance}

We evaluate the read performance of a 32-node Týr cluster and compare it with the results obtained with Rados and BlobSeer on a similar setup. As a baseline, we measure the performance of the same workload on the Azure Storage platform. We preload in each of these systems the whole MonALISA dataset, for a total of $\sim 100$ Gigabytes of uncompressed data. We then perform random reads of 800 byte each from both the raw data and the aggregates, following a power-law distribution to increase read concurrency. This read size corresponds to a 100-minute average of aggregated data. We throttle the number of concurrent requests in the system to a maximum of 1,000.

We plot the results in Figure 9.4. The lightweight read protocol of both Týr and Rados allows them to process reads at near-wire speed and to outperform both BlobSeer and Azure Storage peak throughput by $44 \%$. On the other hand, BlobSeer requires multiple hops to fetch the data in the distributed metadata tree. This incurs an additional networking cost that limits the total performance of the cluster. Under higher concurrency, we observe a slow drop in throughput for all the compared systems except for Azure Storage due to the involved CPU in the cluster getting overloaded. Once again, linear scalability properties of Azure could be explained by the higher number of nodes in the cluster, although this can't be verified because of the lack of visibility into Azure internals. Týr and Rados show a similar performance pattern. Measurements show Rados outperforming Týr by a margin of approximately 7\%. This performance 


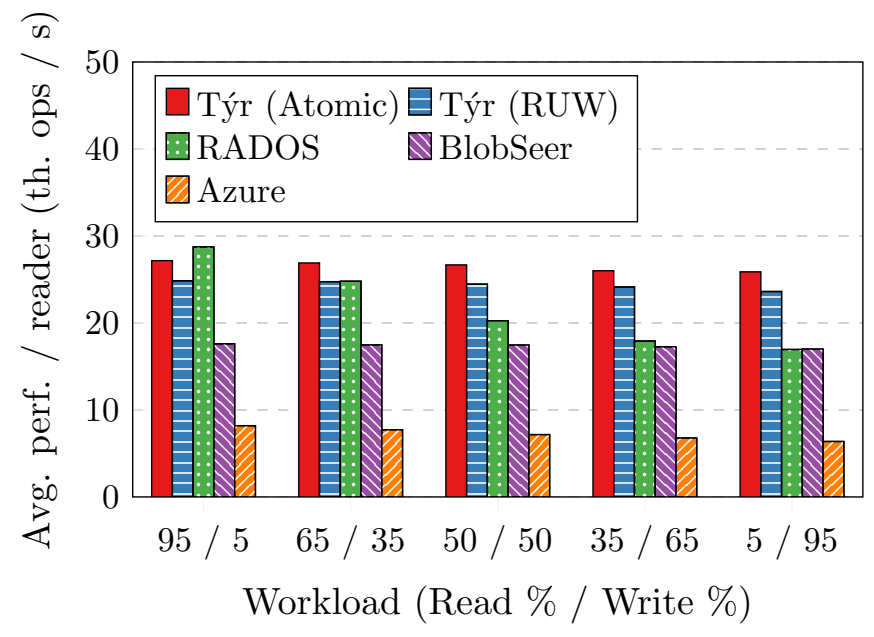

Figure 9.5: Read throughput of Týr, Rados, BlobSeer and Azure Storage for workloads with varying read to write ratio. Each bar represents the average read throughput of 200 concurrent clients averaged over one minute.

penalty can partly be explained by the slight overhead of the multi-version concurrency control in Týr, enabling it to support transactional operations.

\subsection{Reader / writer isolation}

A key, known advantage of multi-version concurrency control is the isolation it provides between readers and writers. We demonstrate this in Týr by simultaneously performing reads and writes in a 32-node cluster, using the same setup and methodology as with the two previous experiments. To that end, we preload half of the MonALISA dataset in the cluster and measure read performance while concurrently writing the remaining half of the data. We run the experiments using 200 concurrent clients. With this configuration, all three systems proved to perform above $85 \%$ of their peak performance for both reads and writes, thus giving comparable results and a fair comparison between the systems. Among these clients, we vary the ratio of readers to writers in order to measure the performance impact of different usage scenarios. For each of these experiments, we monitor the average throughput per reader.

The results, depicted in Figure 9.5, clearly illustrate the added value of multiversion concurrency control on which both Týr and BlobSeer are based. For these two systems, we observe a near-stable average read performance per client despite the varying number of concurrent writers. In contrast, Rados, which outperforms Týr for a 95/5 read-to-write ratio, shows a clear performance drop as this ratio decreases. Azure performance decreases in a similar fashion as the number of writers increases. 


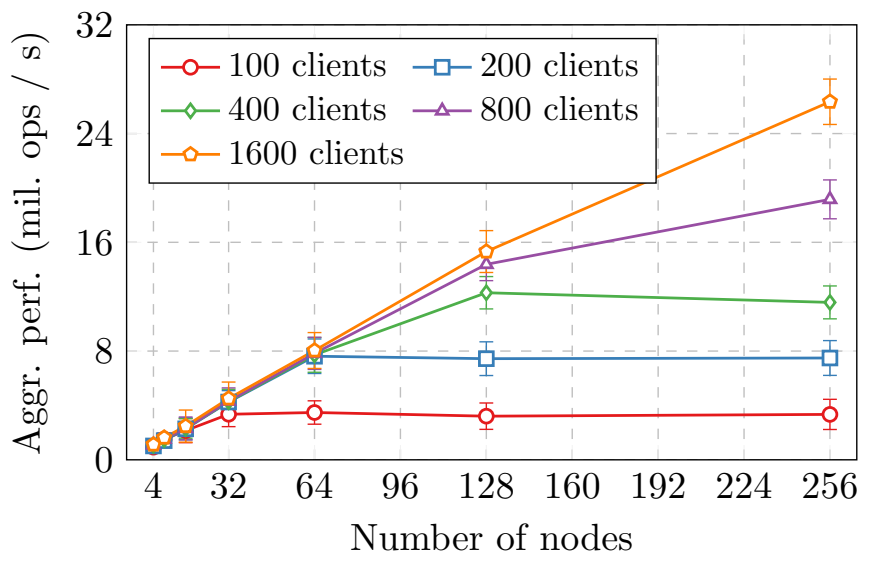

Figure 9.6: Týr horizontal scalability. Each point shows the average throughput of the cluster over a oneminute window with a $65 \%$ read / 35\% write workload, and $95 \%$ confidence intervals.

\subsection{Horizontal scalability}

We test the performance of Týr when increasing the cluster size up to 256 nodes. This results in an increased throughput as the load is distributed over a larger number of nodes. We use the same setup as for the previous experiment, varying the number of nodes and the number of clients, and plotting the achieved aggregated throughput among all clients over a one-minute time window. We use the same $35 \%$ write / $65 \%$ read workload (with atomic transforms) as in the previous experiment.

Figure 9.6 shows the impact of the number of nodes in the cluster on system performance. We see that the maximum average throughput of the system scales near-linearly as new servers are added to the cluster. With this setup, a 256-node Týr cluster peaks at 26.4 million operations per second.

\subsection{Conclusions}

In this chapter, we could demonstrate the effectiveness of Týr in a BDA applicative context. Using Týr as a storage backend for MonALISA leverages write coordination, enabling us to prove the performance and scalability of both transactional operations and atomic transforms. In particular, we highlight the performance of Týr's lightweight transaction protocol which provides very high velocity compared to lock-based solutions while incurring only a small overhead compared to non-transactional systems. We finally note the relevance of the multi-version concurrency control, which enables the readers not to be impacted by a concurrent, conflicting write workload. 


\section{Chapter 10}

\section{Large-scale logging in support of computational steering for HPC}

The use-cases for data logging span beyond system monitoring on BDA. A distributed shared $\log$ is a versatile primitive for a variety of applications. In scientific applications, distributed logs can play many roles such as in-situ visualization of large data streams, centralized collection of telemetry, data aggregation from array of physical sensors or live data indexing.

Distributed shared logs are an especially challenging data structure to implement on common HPC platforms due to the lack of efficient append operation in Lustre (142) or GPFS (140). While part of the POSIX standard, this operation has not been the main focus during the development of parallel file systems. Although application-specific, custom-built solutions are possible, they require a significant development effort and often fail to meet the performance requirements of data-intensive applications running at large scale.

Because of its capability to handle append operations at high velocity, Týr is a good candidate for such applications. The increasing node-local storage capabilities provided by modern, leadership-class supercomputers offer the opportunity to deploy Týr alongside the application in HPC environments. This node-local storage is provided by non-volatile memory (NVM) devices, such as solid-state disks. For example, MareNostrum IV (BSC, 13th of the TOP500) (2) or Theta (ANL, 16th) (77) nodes embedded SSD of respectively 240 and 128 GB. Sierra (4) (LLNL) and Summit (5) (ORNL) will offer 800 GB node-local SSD storage, while Aurora (1) (ANL) nodes will 


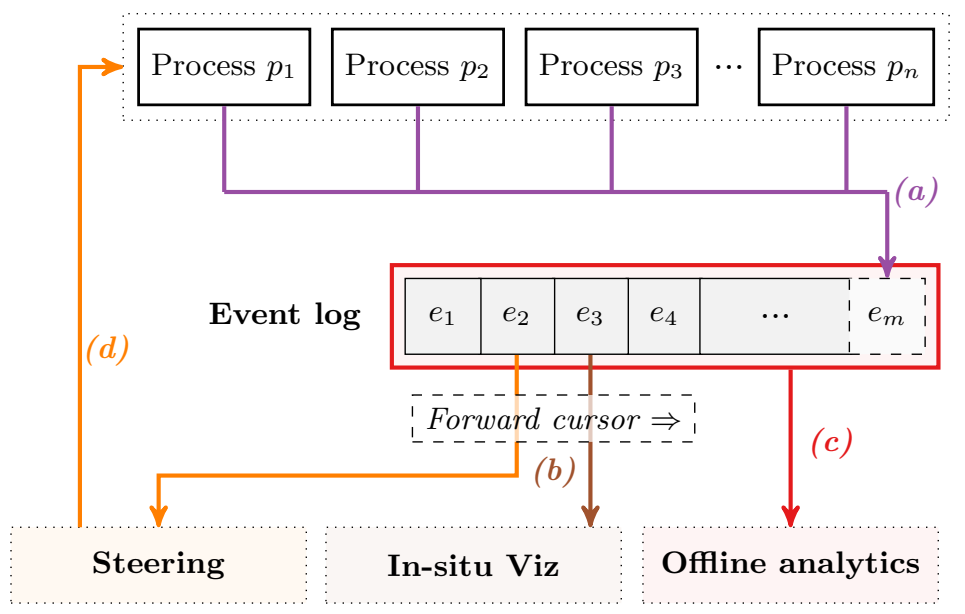

Figure 10.1: Illustration of telemetry event collection. Simulation ranks generate events to a central log (a). Such events are used directly for online tasks such as visualization or computational steering (b), or are stored for further offline processing (c). Computational steering in turn controls the simulation (d).

be equipped with non-volatile random-access memory (NVRAM).

In this chapter we demonstrate the applicability of Týr design in support of largescale data logging on HPC platforms, specifically focusing on telemetry event management. We detail how the design of Týr enables it to scale far beyond the capabilities of traditional file-based storage.

\subsection{Distributed logging for computational steering}

In telemetry event management, the events generated by an application carry data related to its state, its performance, or other application-specific information. Analyzing these events while the application is running can be very useful, for instance to verify that the application behaves as expected, or to run in-situ visualization (57). This information can also be used for in-situ analytics (176) or computational steering (37; 87; 172). The latter can in turn influence the simulation in real-time, e.g. increasing its resolution on a particular zone, or re-running part of it with different parameters. We illustrate this in Figure 10.1.

In this chapter we focus on the application of telemetry event management to computational steering, which presents unique challenges for data storage that we will describe in detail. 


\subsubsection{Required API}

The general API for computational steering is that of a simple multi-producer, singleconsumer distributed log. Multiple ranks generate events that are ingested by the log storage system in parallel. A computational steering process concurrently reads and processes the generated events, extracting the required information to steer the computation in near real-time.

The minimal API we consider in this chapter for computational steering is very simple, being composed of only two operations. All event generator ranks insert events in a shared log using an append function. The computational steering process repeatedly calls a pop method, which iterates the log, returning the next event in the log each time it is called.

\subsubsection{Challenges}

When only offline analytics tasks are necessary, one could think of generating individual logs on each rank, that would be collected and merged once the application has finished. While this solution could be applicable to small scale simulations with low amounts of data generated, it becomes increasingly difficult as the size of the application increases. Running a post-processing step merging data from all ranks into a single log may take a significant amount of time, often more than the simulation itself, and only collecting logs may require a significant development effort.

This approach is simply not possible when using these logs for online tasks such as computational steering, which require to collect logs in real time from all nodes running the simulation. In that case, a naive implementation is to stream all events using MPI (72) to the controller node, but this approach will fail should the event throughput momentarily exceeds the controller capacity, while at the same time tying the simulation to the simulation controller. In such case, a buffer in between the two components helps at the same time mitigate bursting generation rates and decouple the simulation from the control.

In case the data must be used at the same time for multiple purposes, a central log storage simplifies the architecture of the application, consequently requiring less development effort, often increasing performance and simplifying application audit and debugging. While implementing distributed logging on HPC platforms is complicated 
already because of the limitations of traditional file-based storage, scaling it brings additional challenges. Most notably, parallel file systems are well known to be generally bad at handling concurrent, conflicting writes originating from different clients (107, 117; 156; 162). Unfortunately, distributed logs are by design a highly concurrent data structure in which multiple processes essentially compete to write to the tail of a unique object.

\subsection{Experimental setup}

All experiments carried out in this chapter are based on the Theta supercomputer (77), hosted at the Argonne Leadership Computing Facility (ALCF) of Argonne National Laboratory. Theta is a 9.63 petaflops system, ranked 16 in the TOP500 as of June 2017. It is composed of 3,624 nodes, each containing a 64-core Knights Landing Xeon Phi CPU with 16 GB of high-bandwidth in-package memory (MCDRAM), 192 GB of DDR4 RAM, and a 128 GB SSD. Network connectivity is provided by Cray Aries interconnect with Dragonfly configuration.

Theta storage system is based on Lustre. Its 10 TB parallel file system spans 170 storage servers. Theta is also connected to the GPFS file system of the Mira supercomputer (3), co-located in the same facility using high-speed interconnects. This configuration allows for experiments on both Lustre and GPFS from the same experimental platform.

\subsubsection{Evaluated systems}

We compare the performance of Týr with that of Zlog (12), as well as with that of the parallel file systems available on Theta: Lustre and GPFS.

Zlog is a logging system which implements the CORFU (24) log design over the Ceph file system (167). At its core a network sequencer lie, with a simple atomic counter initialized with the size of the file and incremented by the size of each write as data is appended to the log. Multiple such sequencers can be deployed over the network, but one and only one sequencer will be used for each log. We worked with the authors of Zlog to reimplement the sequencer over UDP, which offers orders of magnitude better performance than the original TCP implementation on our experimental platform. 
In addition to Týr or Zlog, we also consider the performance achievable by the parallel file systems Lustre (142) and GPFS (140).

\subsubsection{Platform configuration}

For each application run, we use approximately a 5:1 compute-to-storage core ratio that we empirically determined to be optimal for our setup. Yet, it is highly dependent on this particular setup, and would likely be different in practice. First, Theta's Knights Landing architecture embeds a large number of cores per node. We measured the storage not to be CPU-bound during our experiments. As such, using a lower core-pernode count would have resulted in a higher ratio. Second, our benchmark dedicates all resources to I/O. Any real application would very likely be less I/O intensive and consequently require a higher ratio. We expect the ratio for a real application to be closer to 20:1 - which is for instance the compute-to-storage ratio for Lustre on Theta.

Although we could choose to co-locate the storage system with the application on each machine, we argue that this setup is not ideal for a distributed logging use case. Indeed, the concentration of writes to a small number of objects inherently creates hot spots in the cluster. As such, it is intuitively more interesting to dedicate a smaller number of large machines than a fraction of all machines for storage.

We use a RAMdisk to store the data on each node. We argue that this setup is reasonable as RAM behaves similarly to NVRAM in respect to performance and access times. We expect NVRAM to be increasingly used in the architecture of future machines, which will be the case of Aurora (1). Additionally, Theta compute nodes embed significantly more RAM than SSD space, which would cause the SSD to behave like a RAMdisk thanks to the file system cache.

\subsubsection{Benchmark}

The minimal API required for a shared log is straightforward to benchmark. Indeed, a single operation is performed by all event generator ranks: append. We develop a simple MPI-based benchmark that sequentially generates events at maximum rate from a number of generator ranks. These events are appended to a single shared log using that operation. 


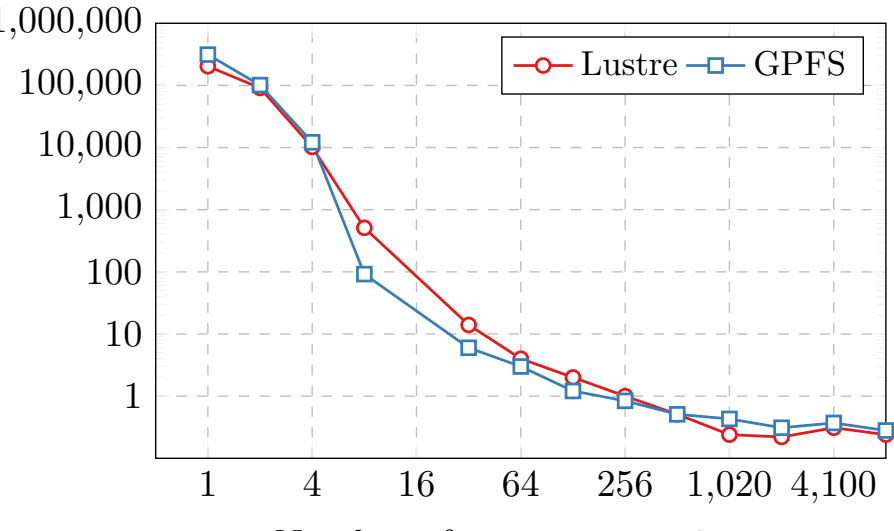

Number of concurrent writers
Figure 10.2: Append performance of Lustre and GPFS on Theta to a single file, varying the number of clients. The performance axis uses a logarithmic scale.

The computational steering process is simulated by a single dedicated rank which reads the logs concurrently, repeatedly calling the pop method. As we only focus on storage I/O, the data is discarded immediately by this process.

Although very simple, we advocate that this benchmark is well suited to evaluate the maximum performance that can be achieved by the system. The append operation causing a very high write contention in distributed logging systems, a large number of process concurrently pushing data at maximum rate to a single log puts a very high pressure on the storage system.

\subsubsection{Evaluating native performance for parallel file systems}

We first performed the preliminary throughput experiments with Lustre and GPFS, allowing us to draw the baseline performance for parallel file systems. We concurrently append to a single file from all ranks. We plot the results in Figure 10.2 . We can see a clear decrease in performance when increasing the process count for both Lustre and GPFS, which behave similarly. Going from one to 8 processes halves the append performance. Using 256 processes yields less than one append per core per second.

A well known methods in HPC system to alleviate this low performance exhibited by most parallel file systems when facing highly conflicting I/O patterns is the use of write proxys. Through this dedicated process all append operations are channeled from the append clients. This eliminates the costly file system access concurrency at the expense of a small increase in append latency. 


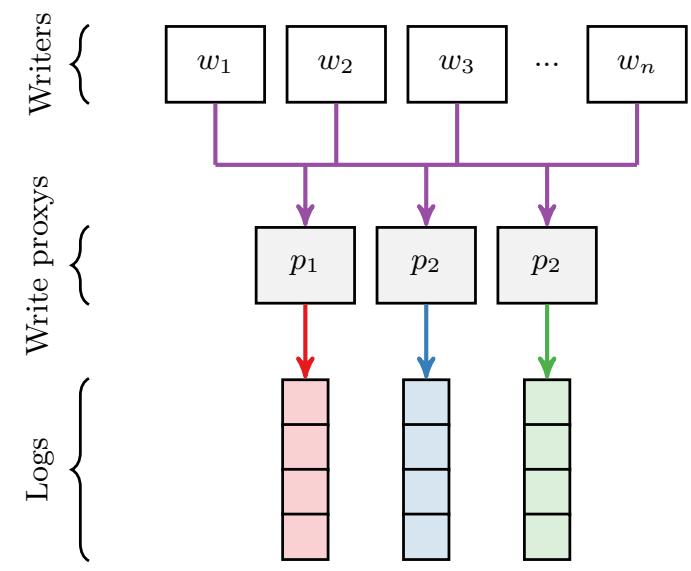

Figure 10.3: Append $\log$ architecture using write proxys for parallel file systems.

The proxy process receives append operations from the append clients. It maintains internally an exclusive cursor on the file that hosts the partition it is associated to, as well as an offset counter. For each append operation, it generates a write to the file at the next free offset along with the current timestamp, and increments the counter by the size of the event.

For this reason, in the rest of this chapter, we channel writes from append clients to the distributed file systems through such a write proxy. We dedicate a write proxy to each different log. This architecture is outlined in Figure 10.3.

In contrast, we evaluate Týr and Zlog without such a process, exposing the storage directly to the append clients. This is because these two systems are designed specifically to handle concurrent, conflicting I/O patterns.

\subsection{Baseline performance}

In this section we seek to show that deploying storage systems such as Zlog or Týr on the compute nodes yields significantly better performance than Lustre or GPFS, even using write proxys. We focus on storing a stream of events generated by a distributed application.

Theta being one of the very few supercomputing platforms offering access to the local SSD drives of the compute nodes, we are able to compare Týr and Zlog on the machines. The application uses between 1 and 8,192 cores as append clients. We deploy Týr and Zlog using between 64 and 1,536 cores for the storage servers, on different machines from the append clients. We measured this ratio to be optimal for 


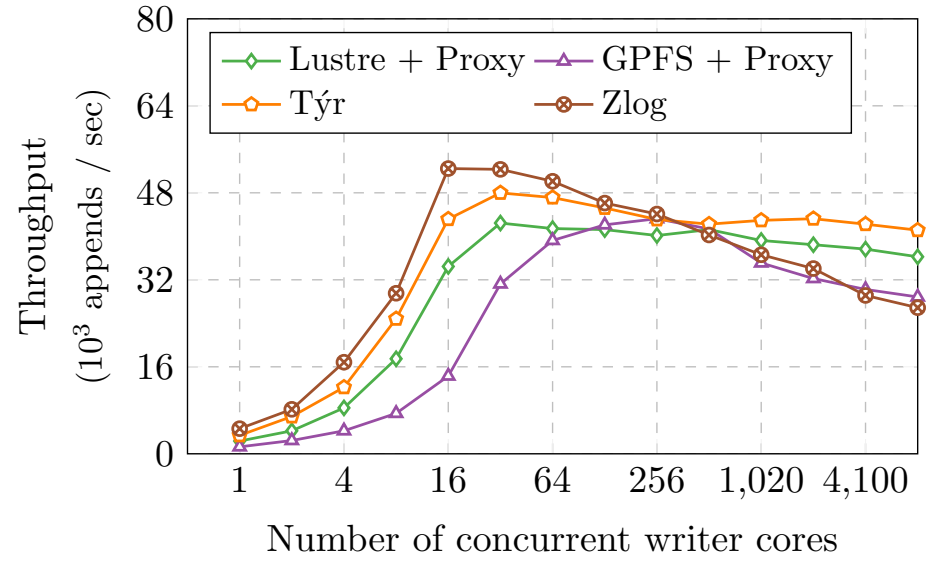

our setup. We measure the number of appends per process per core over 10 minutes. We do not use any write batching or compression. Týr and Zlog are configured with a replication factor of 3 .

We plot the results on Figure 10.4. Overall, they show that the performance achieved by Týr or Zlog is superior to that achieved by parallel file systems when the number of concurrent writers is low. As the concurrency increases, Týr conserves its performance advantage over the other systems. In contrast, the lock-based concurrency control of Ceph limits the performance of Zlog when writer cores are added to the system. Overall, the performance of all systems tend to decrease slightly as more writers are added, because of the increasing write contention at the tail of the log. For Lustre and GPFS, this is also due to the saturation of the proxy node.

\subsection{Further increasing append performance by increas- ing parallelism}

One common method to increase throughput on HPC platforms is to leverage multiple independent files. In this model, each writer rank is assigned to an I/O group. Each group is assigned one or more files, that it accesses independently. This method improves performance as a single file is shared by a lower number of nodes, consequently reducing access concurrency. Such setup is illustrated in Figure 10.5. State of the art streaming data systems on clouds such as Kafka (89) or BookKeeper (80) leverage a similar level of parallelism, splitting each log into a variable number of partitions. 


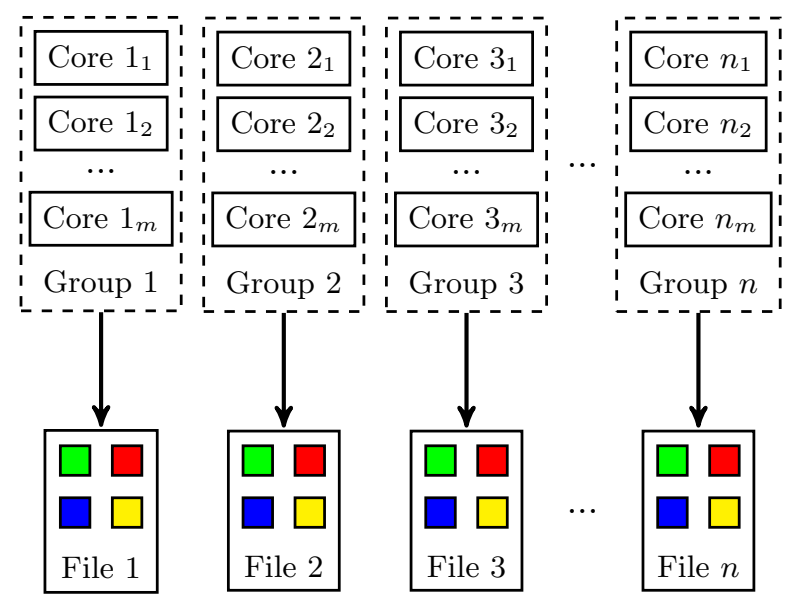

Figure 10.5: Illustration of the multiple independent files method, where each output $\log$ is independently mapped to a group of nodes.

In this model, when a writer generates an event, it is directed to one and only one partition. Each partition hence receives only a subset of all append operations. A partition is mapped to a single object (i.e., blob for Týr, log for Zlog, file for Lustre and GPFS). The possible write throughput for a partition is hence determined by the maximum throughput the underlying storage system can handle for a single object. Increasing the number of partitions for a log increases the total throughput supported by the $\log$ by increasing write parallelism.

In this section we seek to understand how adding partitions affects the append performance in a single log. We perform the same experiment as in the previous section, increasing the number of partitions in the log. We use a dedicated proxy process per partition for Lustre and GPFS.

In Figure 10.6 we plot the results. We show how increasing the number of partitions impacts the performance of the storage. We use the same setup as in the previous section with 8,192 clients, varying the number of partitions between 1 and 64 . The results show a clear increase of the throughput per client. As expected, doubling the number of partitions roughly doubles the achieved throughput on the log by evenly splitting the load across multiple files, reducing hot spots at the tail of the log and consequently increasing throughput. The results show a clear advantage for Týr over other storage systems, consistent to what our observations from the previous section.

We also measure the achieved read performance during this experiment. We did not note any substantial performance degradation when increasing the number of partitions, with only a 5,3\% performance drop between 1 and 8,192 partitions. 


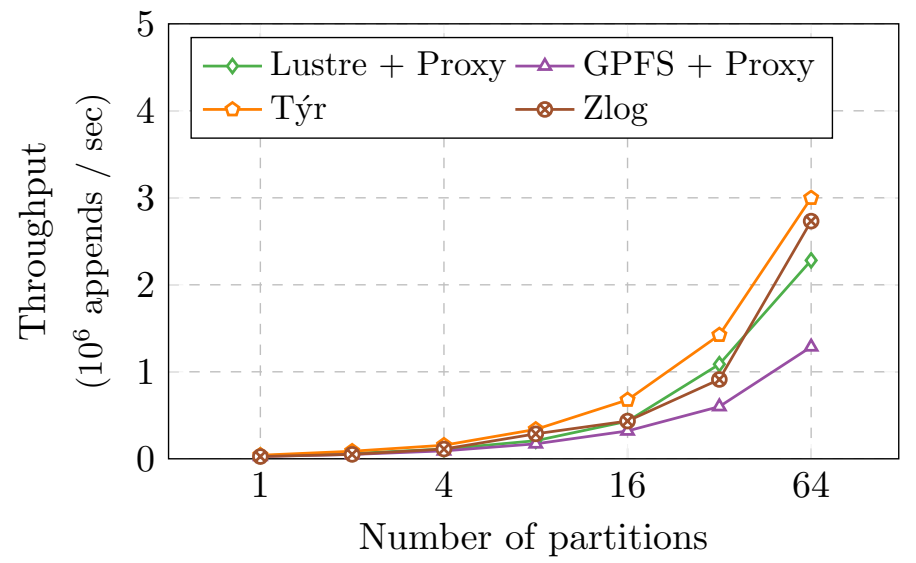

Figure 10.6: Baseline append performance, using up to 8,192 append client cores, with $1 \mathrm{~KB}$ appends.

\subsection{Scaling distributed logging up to 120,000 cores}

Leveraging the findings made in the previous section, this section is dedicated to validating this knowledge at scale by applying it to distributed logging. We experiment using up to 100,000 (1563 nodes) client cores and 20.000 storage cores (313 nodes).

This scale roughly corresponds to half the size of Theta. We use the same append benchmark as in the previous sections. We use 2 output logs for every storage node. We proved this setting to be reasonable in Section 10.4 . We vary the number of client cores from 12,500 to 100,000. Based on our previous experiments, 16 client cores per proxy yield best overall performance. We hence vary the number of partitions proportionally between 780 and 6,250. We use both Týr and Lustre in this experiment, using the same configuration as in the above section. Týr is deployed on 20,000 storage cores. We measure the aggregated throughput achieved in append operations per second across all clients.

Overall, the results depicted in Figure 10.7 confirm the near-horizontal scalability that can be achieved by increasing the number of concurrent partitions, using either file- or object-based storage as backend. We measured a throughput of up to 174 million appends per second on Lustre, and up to 241 million appends per second with Týr.

These results confirm that our findings from the previous section can be applied to achieve near-linear scalability of distributed logging on a high-end supercomputing platform. We argue that achieving such scalability using only the parallel file systems available on this platform would have been extremely hard if not impossible, and likely would have resulted in a significant development effort. While we did not perform 


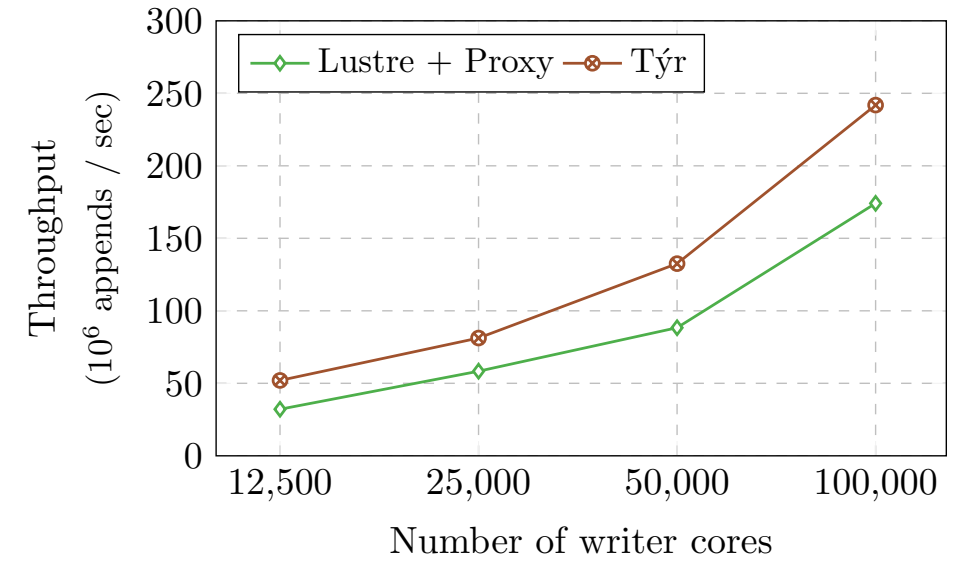

experiments at larger scale for allocation constraints, all the data seems to indicate that further scaling distributed logging should be possible using this setup.

\subsection{Conclusions}

In this chapter, we have demonstrated the relevance of the Týr design for distributed logging at very large scale. Indeed, the lock-free design of the storage system provides sustained write performance even when facing extreme concurrent writes compared to lock-based storage systems such as Zlog + Ceph. In particular, we could scale the system up to 241 million operations per second using 20,000 cores for the Týr storage cluster, coping with the events generated by 100,000 client ranks. 


\section{Chapter 11}

\section{Týr for HPC and BDA convergence}

In this chapter we demonstrate the effectiveness of Týr's key design principles in support of legacy HPC and BDA applications. This evaluation leverages the same experimental platform and configuration as in Chapter 5, adding Týr as a competitor for BlobSeer, Rados and CephFS.

This chapter focuses on non-transactional performance, and seeks to prove that the performance of Týr is competitive with, or even exceeds that of other state-of-the-art storage systems even when such transactional semantics are not needed. This is the case for the set of legacy applications we introduced in Chapter 5.

In particular, using exactly the same experiments and configuration as in Chapter 5 , we seek to understand how Týr behaves compared with its blob storage competitors. For HPC applications, we also provide an overview of the horizontal scalability of Týr. We replicate the above experiments on up to 2,048 storage cores and 6,144 application ranks on a high-end, leadership-class supercomputer hosted at Argonne National Laboratory.

\subsection{Týr as a storage backend for HPC applications}

In this section we add Týr as a backend storage system for BLAST, MOM, ECOHAM and Ray Tracing applications, and compare its performance with that of Lustre, BlobSeer and Rados. 


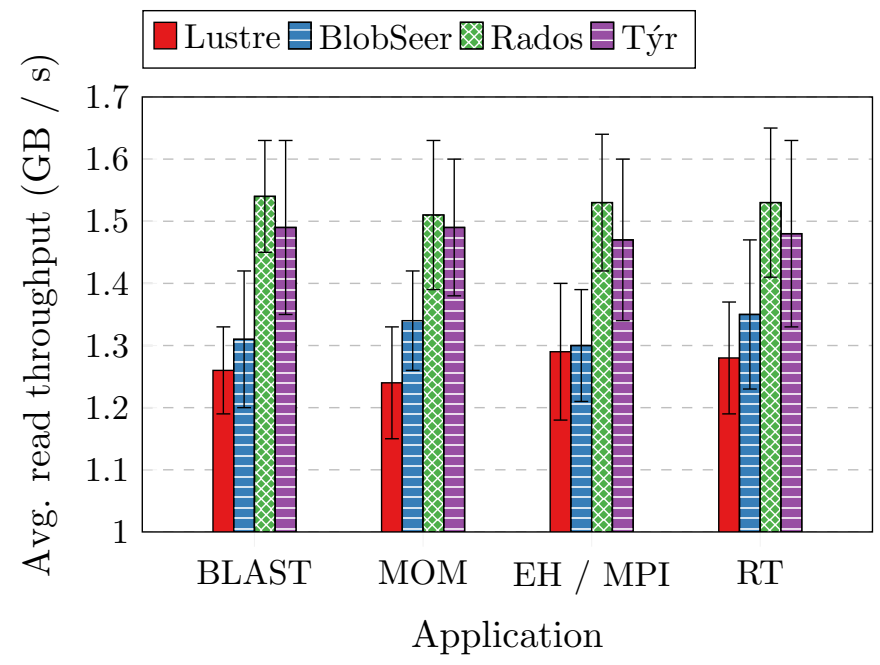

Figure 11.1: Comparison of read throughput for each HPC application with Lustre, BlobSeer and Rados, with $95 \%$ confidence intervals.

\subsubsection{Testbed performance}

In Figure 11.1 we plot the aggregate read throughput for all storage systems. For all configurations, we note that Týr's performance significantly exceeds that of BlobSeer. This is mainly because of its two key features: its more efficient metadata management and predictable data distribution significantly cut access performance, which in most cases do not need to hit any metadata service. While Rados keeps a slight performance advantage over Týr in all cases, the average difference is relatively small (less than 5\% average difference). This difference is mainly due to the cost of multi-version concurrency control, and of the more complex write path required for offering transactional semantics in Týr.

Figure 11.2 shows the write performance achieved by Týr compared with other systems. As for the read throughput, Týr outperforms BlobSeer because of its simpler metadata management scheme. Interestingly, for Ray Tracing, Rados performace drops behind that of all other systems because of the high lock contention resulting from the high write access concurrency. For this application, Týr shows a significant performance advantage for this balanced workload thanks to its more efficient protocols and metadata management, which at the same time isolates readers for writers and provides non-blocking, concurrent write operations.

We plot in Figure 11.3 the resulting application completion improvement. Consistently with the read and write throughput, Týr outperforms BlobSeer in all cases. Ray 


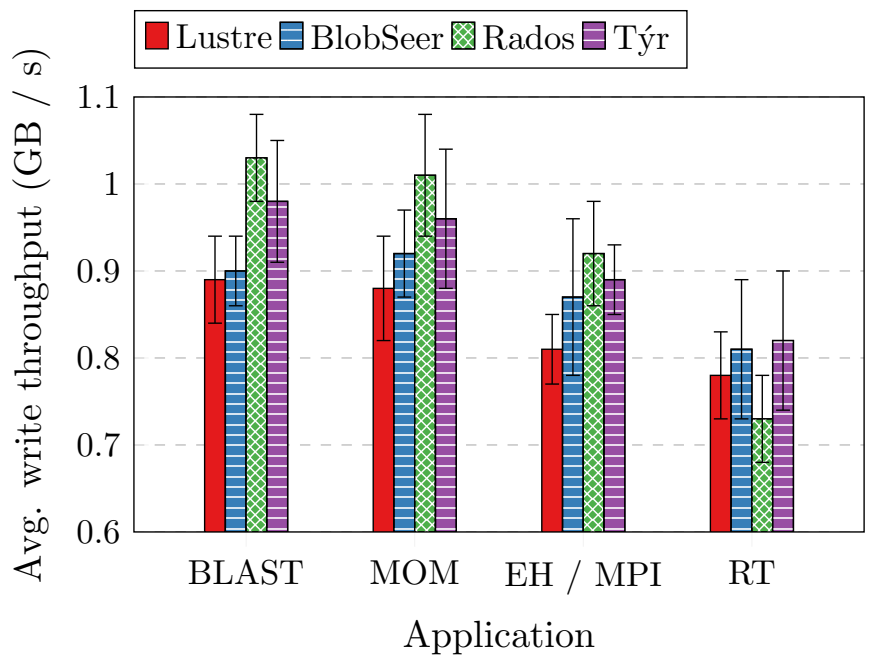

Figure 11.2: Comparison of write throughput for each HPC application with Lustre, BlobSeer, and Rados, with $95 \%$ confidence.

Figure 11.3: Average performance improvement relative to Lustre for HPC applications using blob-based storage, with $95 \%$ confidence.

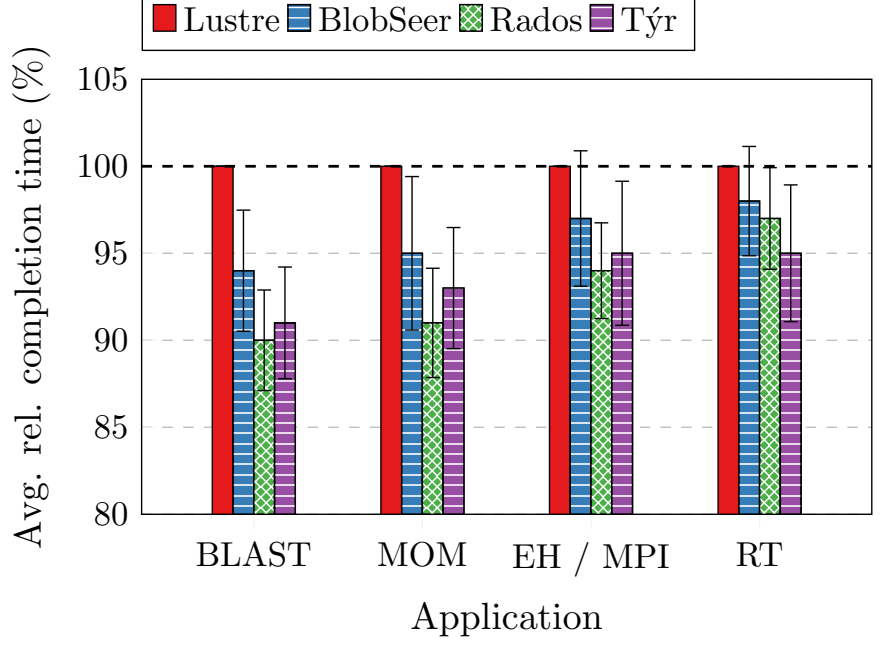

Tracing being the most write-intensive application, the efficient design of Týr gives it a slight $2 \%$ completion time improvement compared to Rados.

\subsubsection{Replicating the results on a high-end platform}

In this section, we seek to prove that the results obtained in the previous section are reproducible on a high-end supercomputer. To do so, we leverage the Theta supercomputer hosted at Argonne National Laboratory, and run the same experiments as in the aforementioned section. Although arguably not an easy task due to the strong limitations of the platform and the lack of superuser rights, blob storage systems such as Týr and Rados can be deployed on such platform. 


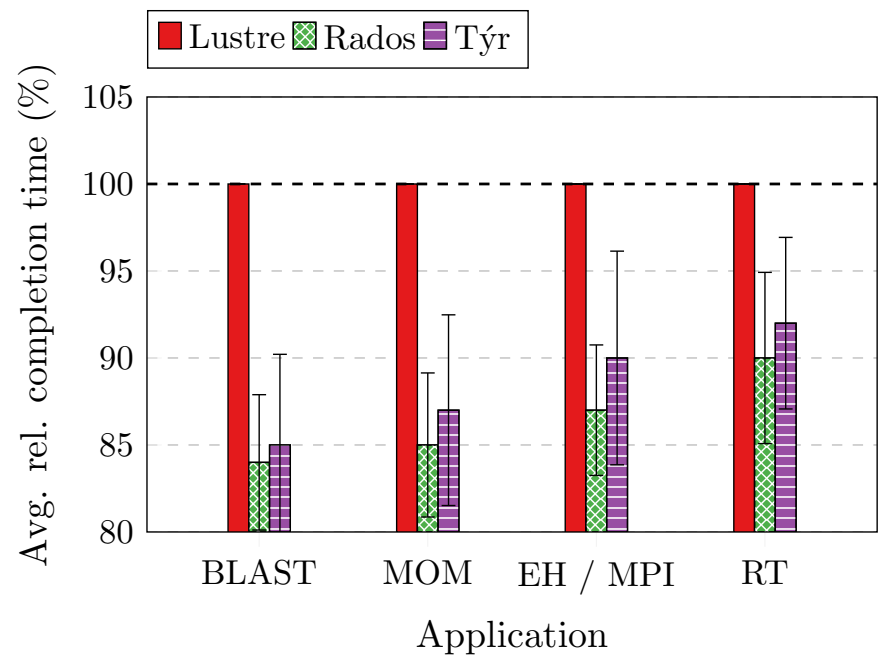

Figure 11.4: Average performance improvement relative to Lustre for HPC applications using blob storage, with $95 \%$ confidence on Theta.

We deploy the applications as described in the previous section, using 32 nodes (totaling 2048 cores), using 24 nodes for computation and 8 nodes for storage, and measure the completion time for each application. Experiments with Lustre were using the file system available to the computer, totaling 170 storage nodes and shared across all users.

We plot the results in Figure 11.4. We show the performance improvement to be significantly higher than on our testbed. The reason for this performance increase is to be found in the technical characteristics of Theta, which offers significantly more RAM than SSD space. As such, for the most part, the storage systems deployed on these nodes behave as in-memory storage systems. We acknowledge that the setup of this platform is particular in this regard and by consequence that the results are not representative of those of another platform with a different setup. Yet, we advocate that the results demonstrate that deploying blob storage systems on high-end HPC platforms is possible without requiring any application modification. We observe a higher variance in the results compared to Grid'5000, which we attribute to the shared nature of the centralized storage.

In Figure 11.5 we scale all four applications on up to 128 nodes of Theta, or 8192 cores. We notice a near-linear decrease of computation time as the size of the cluster increases. With applications such as ECOHAM or Ray Tracing, the performance improvement is slightly lower than with purely read-intensive applications due to the significantly higher cost of write operations compared to read operations. 


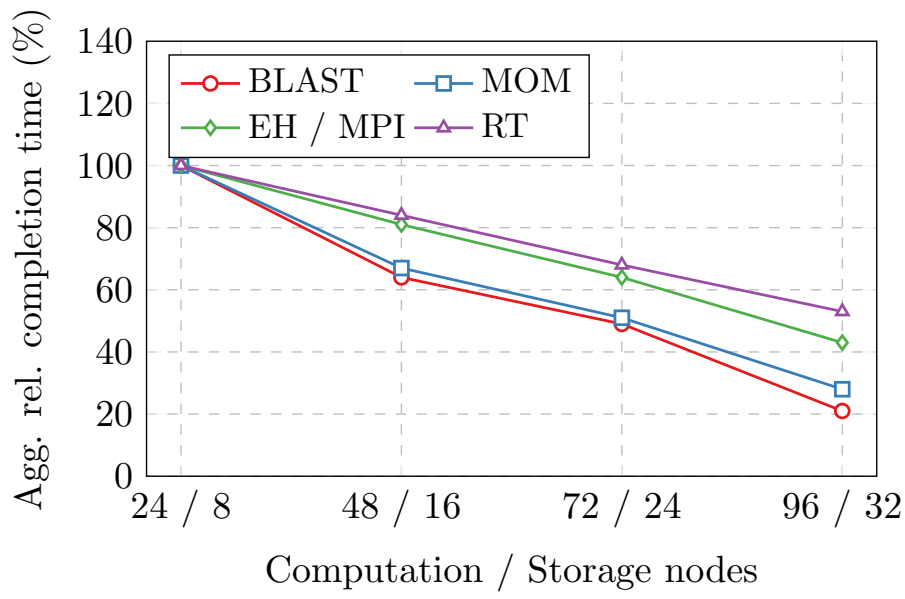

Figure 11.5: Average performance improvement at scale relative to 32 nodes setup for HPC applications using blob-based storage on Theta.

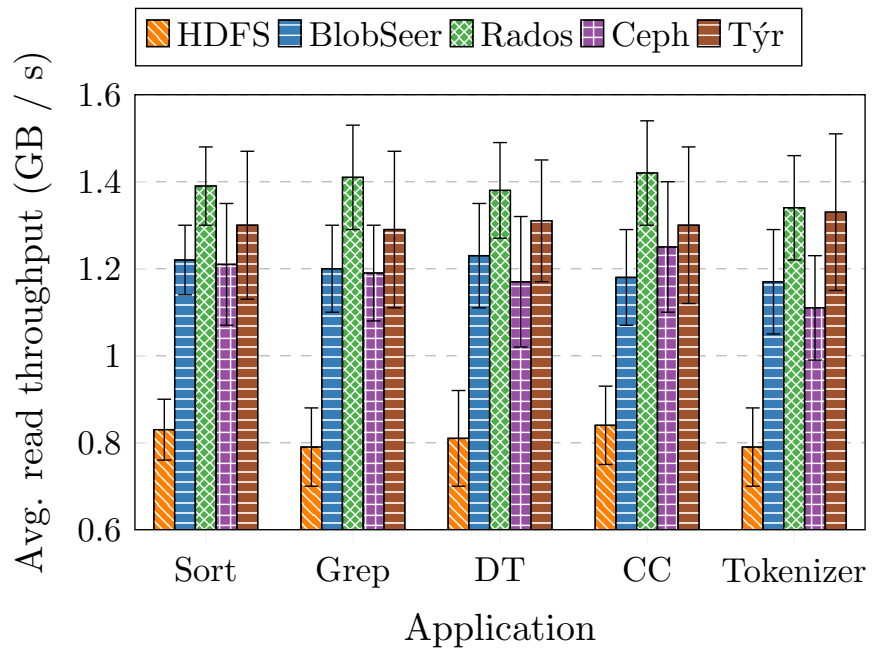

Figure 11.6: Comparison of read throughput for each Big Data application with HDFS, BlobSeer, Týr, Rados and CephFS, with $95 \%$ confidence intervals.

\subsection{Týr as a storage backend for BDA applications}

Figure 11.6 shows the average read bandwidth for these applications. Týr constantly outperforms both Ceph and BlobSeer in all cases, which is due to the direct read capability it shares with Rados. Our measurements during these experiments demonstrate that over $98 \%$ of the read operations performed by Spark could be served by the direct read protocol. The remaining $2 \%$ were served by the multi-chunk read protocol. Yet, similarily to what we could observe with HPC, Týr performance is slightly lower than Rados performance due to the cost of the additional consistency guarantees it provides.

Figure 11.7 highlights the benefits of multi-version concurrency control. The throughput achieved by Týr is less dependent on the application I/O profile than that of HDFS, 


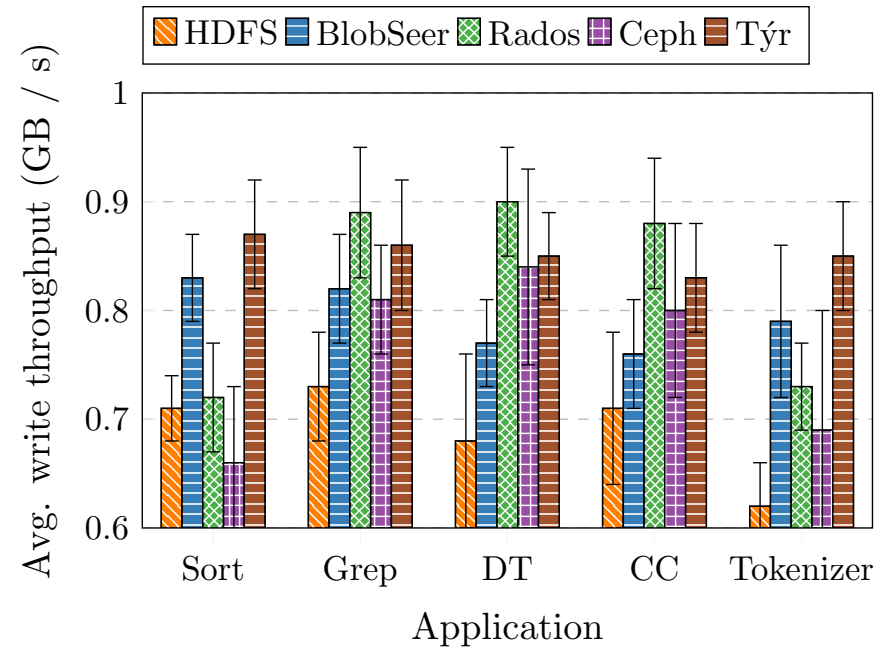

Figure 11.7: Comparison of write throughput for each Big Data application with HDFS, BlobSeer, Týr, Rados and CephFS, with $95 \%$ confidence intervals.

Ceph or Rados. While Týr is outperformed by Rados in read-intensive applications, the situation is inverse for write-intensive applications. With the tokenizer application, Týr throughput is over $15 \%$ higher than that of Rados. On the opposite, Týr's throughput is only $5 \%$ lower than that of Rados with read-intensive applications. In this experiment also, Týr outperforms BlobSeer in all cases because of its more efficient metadata management scheme.

In Figure 11.8 we plot the relative improvement in the total application completion time, diluted in computation. As a result of the previous observations with read and write performance, Týr globally enables significantly lower application completion time compared to Ceph and BlobSeer for all applications. Due to its globally higher write performance with highly concurrent workloads, Týr brings substantial application completion time reduction for write-intensive applications such as Tokenizer, while causing a very slight completion time increase when reads are frequent.

\subsection{Conclusions}

In this chapter, we have drawn a baseline performance for Týr using legacy applications initially designed for file-based storage. Overall, we prove that the design of Týr enables it to significantly outperform both BlobSeer and traditional file systems in all cases, for both HPC and BDA applicative use-cases. This is due to its design, leveraging nonblocking writes thanks to multi-version concurrency control and direct writes using consistent hashing techniques. 


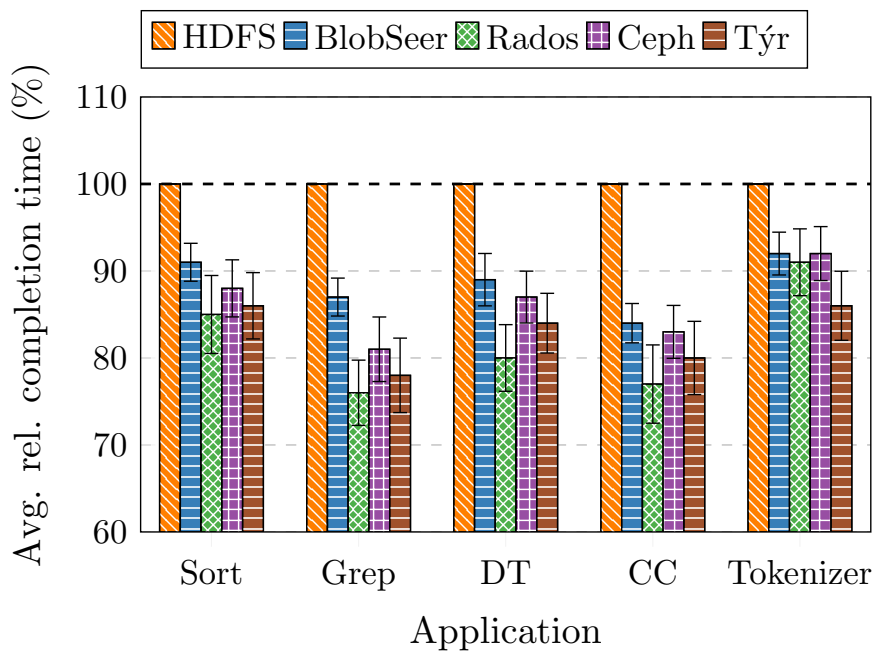

Figure 11.8: Average performance improvement relative to HDFS for Big Data applications using blob-based storage, with $95 \%$ confidence intervals.

Týr performance shows a small penalty compared to that of Rados, except for write-intensive applications for which multi-version concurrency control is particularly efficient. This lower performance is the result of the higher consistency guarantees of Týr, providing additional data consistency guarantees thanks to its fully transactional API. Legacy applications such as the ones used in this test are not designed to take advantage of these features. The previous chapters factually demonstrated how these features can further highlight the relevance of Týr design in the context of purpose-built applications. 


\section{Chapter 12}

\section{TýrFS: Building a file system abstraction atop Týr}

In Chapters 5 and 11 we did demonstrate that blobs can cover a large part of the POSIX I/O API of a file system while dropping features that are not necessary for a wide range of applications. Yet, a potentially large number of legacy applications not relying on an I/O or data processing framework such as MPI-IO or Spark may rely on POSIX I/O operations not supported by blob storage systems. This is notably the case of directory operations.

While legacy applications could be rewritten to take advantage of blob storage, we acknowledge that it is not reasonable to propose a storage solution replacing file-based storage that would break compatibility with these legacy applications. Ideally, blob storage would integrate seamlessly in existing storage infrastructures for both HPC and BDA, supporting at the same time legacy applications relying on strict POSIX I/O semantics and newer applications taking advantage of the performance improvements permitted by blob storage. Furthermore, this transition should not hinder the performance of these legacy applications.

Ceph (167) has demonstrated that blobs are a strong building block upon which a file system can be efficiently built. Indeed, it leverages Rados (169) as its storage layer. Yet, the lack of transactional semantics in the latter cause Ceph to drop important consistency guarantees (13), which can lead to data corruption under concurrent workloads. In contrast, Týr natively provides these transactional semantics. In this chapter, we prove that such features enables the development of a POSIX I/O compliant 
file system that provides high throughput while retaining important data consistency guarantees.

A particular and hard problem we focus on while developing this file system layer is the management of very small files. Indeed, workloads composed of very small files becomes increasingly important for a range of applications processing data from Internet of Things (IoT) applications, generated by data-intensive applications such as CM1 (36) or HACC (74), or processed by large scale workflows such as Montage (27), CyberShake (69) or LIGO (15). While a wide range of work tackles this issue, the optimizations proposed in the literature having a significant impact on read performance rely on archiving, and optionally indexing files. Such approaches are indisputably effective for analyzing large sets of data, they invariably result in dropping a significant part of the POSIX I/O API. Notably, no related work considers modifying a file after

it has been written. Instead, we advocate that the direct read capabilities of Týr make it a strong building block for such a file system.

In this chapter, we first describe the additional challenges associated with managing small files. We factually demonstrate why the existing approaches fail to provide adequate performance with such workload, and how Týr data management comes to the rescue with simple design principles. Based on a prototype implementation of a file system called TýrFS and leveraging these principles, we demonstrate experimentally the relevance of the design for efficient management for small files.

\subsection{Motivation: the overhead of reading small files}

We first describe in more detail the issue by studying experimentally the concrete performance effects of reducing file size with a large data set. For that matter, we plot the time taken and file system CPU usage when reading a constant-size data set while decreasing the size of the files it contains.

We perform these experiments using both the Lustre (142) (2.10.0) and OrangeFS (123) (2.9.6) distributed file systems, which we setup on 24 nodes of the Paravance cluster in Rennes (France) of the Grid'5000 testbed (30). On 24 other machines of the same cluster (384 cores) we launch a simple ad-hoc MPI benchmark reading in parallel the contents of a pre-loaded set of files. The set size varies between $38,400 \times 32 \mathrm{MB}$ files 

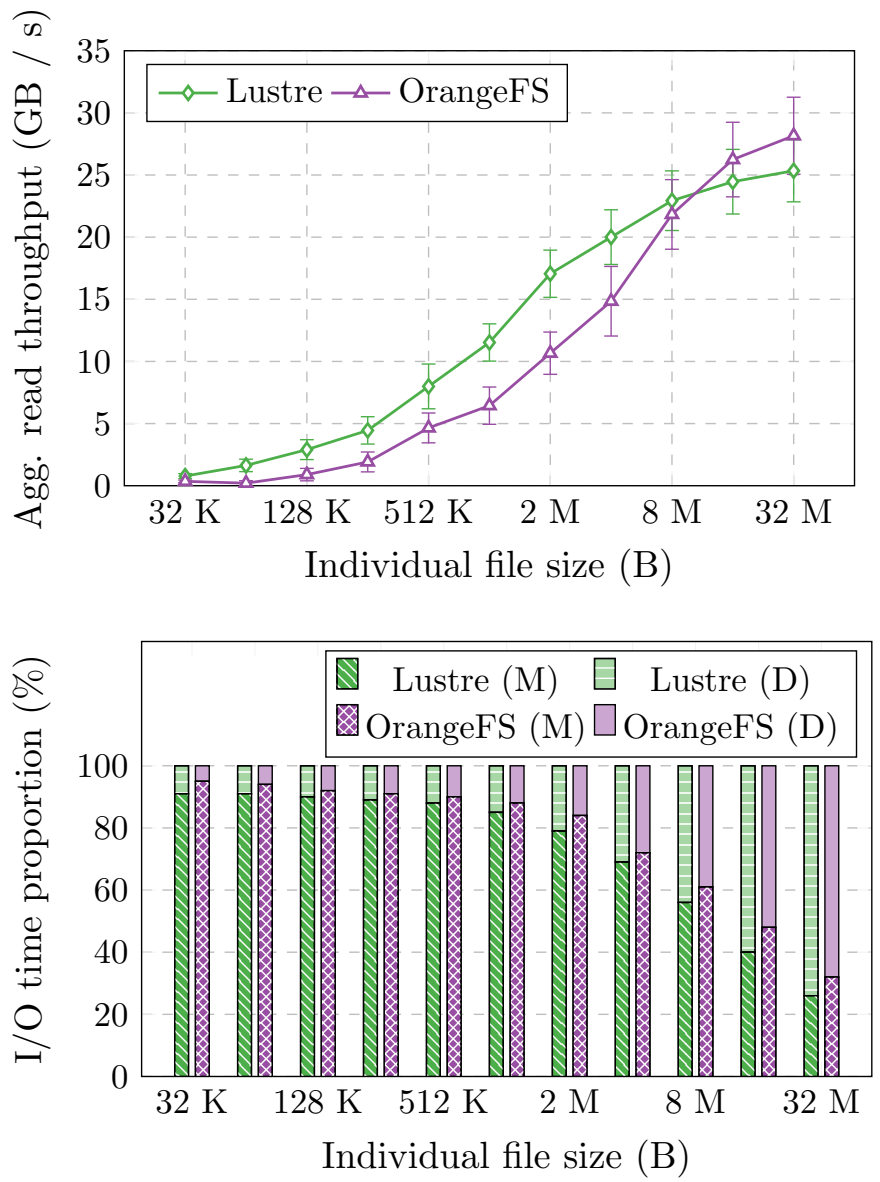

Figure 12.1: Lustre and OrangeFS read throughput for a fixed-size data set when varying the size of the individual files it is composed of.

Figure 12.2: Breakdown of reads between metadata (M) and data (D) access for a fixed-size data set varying the size of the individual files it is composed of.

(100 files per rank) to $3.84 \times 10^{7} \times 32 \mathrm{~KB}$ files $(100,000$ files per rank), for a total combined size of 1.22 TB.

In Figure 12.1 we plot the aggregate throughput achieved by Lustre and OrangeFS. For both systems we note a decrease of the read throughput as we reduce the size of the files. The decrease is as significant as one order of magnitude when replacing 32 MB by $32 \mathrm{~KB}$ files.

In Figure 12.2 we detail these results. We calculate the total execution time of the benchmark with Lustre and OrangeFS. We highlight the proportion of this time spent on the open operation fetching the metadata (M) and on the read operation actually reading the data (D). Interestingly, with smaller file sizes, the open operation takes a significant portion of the total I/O time. This proportion is above $90 \%$ for $32 \mathrm{~KB}$ files for both systems, while it is less than $35 \%$ for larger file sizes.

These results clearly demonstrate that reducing the size of the files puts a very high 


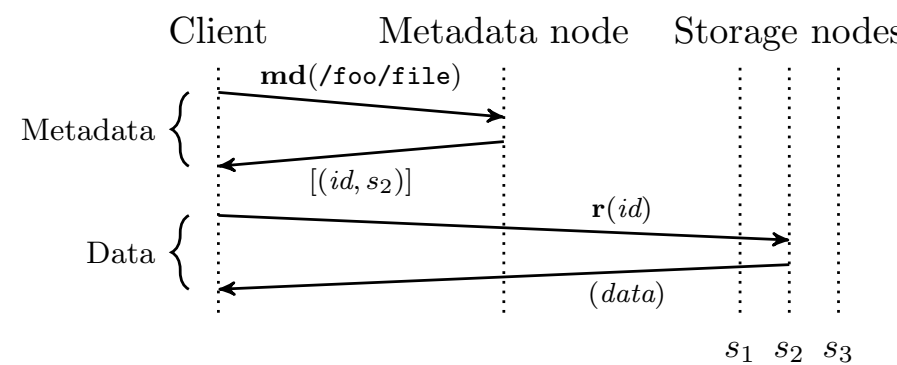

Figure 12.3: Typical read protocol for a small file with centralized metadata management (e.g. Lustre or HDFS).

pressure on the file system metadata. This is caused by the very architecture of most file systems, in which metadata and data are separated from each other. This implies that metadata needs to be accessed before performing any read operation. Consequently, we argue that reducing the cost of metadata management without changing the file system architecture cannot possibly address the root cause of the performance drop.

These observations guide the design and implementation of TýrFS, which we detail in the following sections.

\subsection{TýrFS design}

In this section we detail the main architectural and design goals that drive the design of a file system optimized for small files. We consider as small files those for which it does not make sense to split them into multiple pieces (chunking). Since this notion is variable and cannot be defined precisely, we will consider as small - files with a size under a few megabytes.

\subsubsection{Detailing the metadata distribution issue}

Let us consider the behavior of a typical distributed file system for reading a file, whose path is /foo/file. We consider the case of centralized metadata management, which is the base architecture of Lustre (142) or HDFS (164). As shown in Figure 12.3, the request is performed in two steps. First, the client interrogates the metadata server to get the layout information of the file to read, including the actual node on which the data is located. Only with this information the client can access the data in a second step. While the cost of such metadata fetching is amortized when reading large files, it cannot be with small ones. Should the user seek to read many files, the cost of this 


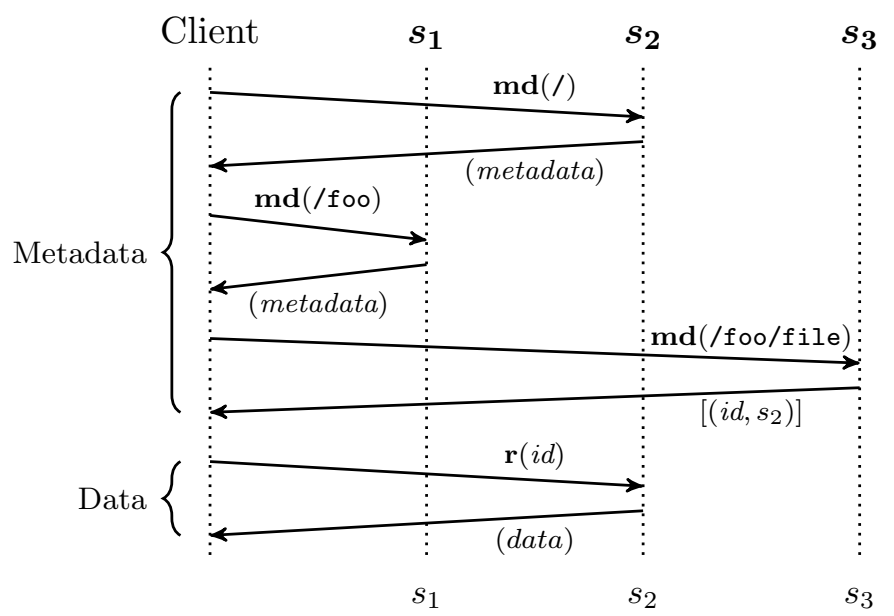

Figure 12.4: Typical read protocol for a small file with decentralized metadata management on three storage nodes $s_{1}, s_{2}$ and $s_{3}(e . g$. OrangeFS).

communication with the metadata node is to be paid for each file access. This largely explains the results observed in Section 12.1 .

The previous results also noticeably show a further performance degradation with OrangeFS (123) compared to Lustre. This is caused by the distribution of the metadata across the entire cluster, which brings better horizontal scalability properties at the cost of additional metadata requests, as illustrated in Figure 12.4. The location of the metadata for any given file in the cluster is independent from the location of its data.

Intuitively, we understand that two problems are to be tackled when dealing with small files at scale:

- First, the client should be able to locate by itself the pieces of data to be read without requiring communication with a dedicated metadata server. This is similar to the direct read principle of Týr.

- Second, all the metadata required to read a file should be colocated with the file data whenever possible.

These two steps would allow the client to bypass metadata requests entirely, consequently offering significantly better performance when dealing with many small files. While these principles are seemingly easy, they are challenging to implement for distributed file systems because of the hierarchical nature of their namespace. 


\subsubsection{Data distribution using consistent hashing}

In typical file systems, the data is distributed across the cluster in a non-predictable fashion. A metadata process is responsible for assigning a set of nodes to each file based on some system-specific constraints (91). This location is saved in the file metadata, which therefore needs to be queried in order to later pinpoint the location of the data in the cluster. While this allows a great latitude in finely tweaking data location, it inherently imposes at least two successive requests in order to retrieve data in the cluster. As the data is small, this request cost is hardly amortizable for small files.

In contrast, the predictable data distribution that is key to the design of Týr enables certain read requests to entirely bypass any metadata node. Such predictable data distribution is however challenging for file systems due to their hierarchical nature. For accessing any single file, a client needs to read the metadata of all the folder hierarchy for this file in order to check, for example, that the user has the correct permissions for reading it. Let us illustrate this with a simple example. A folder at /foo contains many files to be processed by a data analytics tool. To distribute the load across the storage nodes, these files should arguably be spread across the cluster. As such, while clients would be able to locate each file in the cluster because of the predictable location algorithm, they would still need to access the metadata for /, /foo and for the file to be read. All these metadata pieces are potentially located on a different set of servers, and would require additional communication that we are precisely seeking to eliminate.

Although such predictable data distribution is a step towards the solution, it cannot be considered independently of metadata management. Some file systems such as Ceph (167) partially leverage predictable distribution for reducing the size of the metadata without addressing the issue of its distribution.

\subsubsection{Dynamic replication for metadata management}

To significantly reduce the overhead of metadata fetching we propose to collocate it with the data it concerns. Let us keep our example of the / foo folder containing many files. This implies that the node storing the data for the file /foo/a.txt would also hold the metadata for /, /foo and /foo/a.txt. We do so by leveraging an architecture roughly similar to that of OrangeFS. Each node composing the file system cluster integrates two services. First, a metadata service manages meta-information relative to folders and 
files. Second, a data service persists the data relative to the objects (files or folders) stored on this node.

Data and metadata colocation: As a first principle, we propose to always store the metadata for any given file on the same nodes the first chunk of the file is stored. As small files are by definition composed of a single chunk, this effectively eliminates the need for remotely fetching the file metadata before accessing its data. This contrasts with the design of OrangeFS, which does not guarantee such collocation. Such design is conceptually similar to the decision of embedding the contents of small-enough file directly inside inodes on the Ext4 local file system (40).

Colocating the file data with the metadata of its folder hierarchy is a significantly harder problem. Indeed, a folder may contain many other folders and files potentially stored on different nodes. Specifically, the root folder itself is by definition ancestor of all folders and files. One could think of statically replicating this data to every relevant node. Yet, this solution would have huge implications in terms of storage overhead because of the potentially large amount of metadata replicated, and in terms of write performance because of the need to maintain consistency across all metadata replicas.

Dynamic metadata replication: To alleviate this overhead, we replicate the metadata dynamically. The metadata agent on each node determines which of the metadata to replicate locally based on the current workload it serves. This solution is largely tackled in geo-distributed storage systems, in which dynamic replication is used to selectively replicate data objects to various discrete regions while minimizing write and storage overhead by adapting this replication based on user requests $(82$; 112 ; 116). Our solution is largely inspired by this proved design and set of best practices.

Reducing the metadata overhead: We further reduce the storage overhead by only replicating the metadata that is strictly necessary for accessing a file. Indeed, only a tiny subset of the whole metadata for the folder hierarchy of a file has to be accessed before reading it: permissions. In addition, we argue that permission changes are a very infrequent operation that is performed either when a folder is first created or as a manual management task which is unlikely to be time-sensitive ; we could not find any application in which permission change is in the critical path. As such, replicating the permissions is unlikely to have a significant negative effect on the performance of real-world applications. Intuitively, this solution implies that the closer a folder is to 


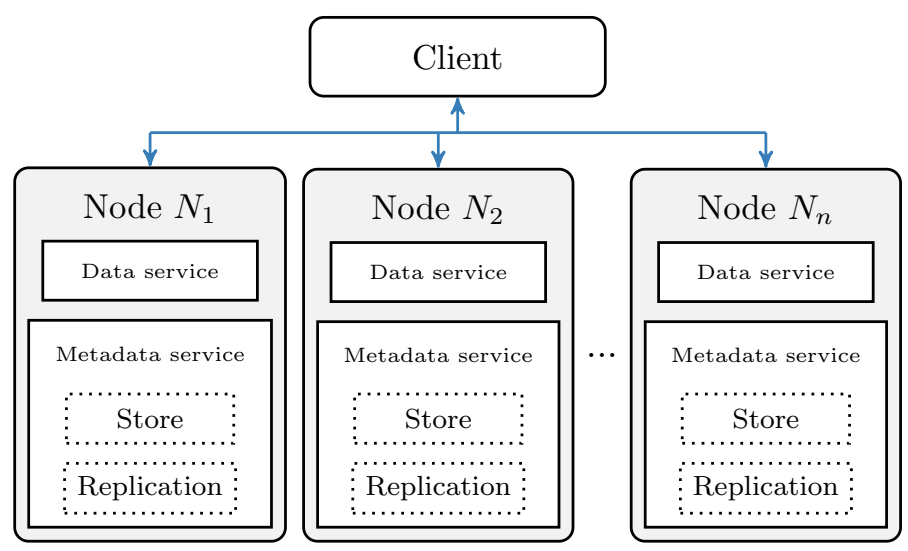

Figure 12.5: High-level overview of the proposed TýrFS architecture. Each node integrates both a data and a metadata service. The metadata service itself is composed of a local metadata store and the dynamic metadata replication service.

the root of the hierarchy, the more likely its permissions are to be replicated to a large number of nodes. As an extreme case, the permissions for the root folder itself would be replicated to every node in the cluster.

In Figure 12.5 we show a high-level overview of the proposed architecture, highlighting the different modules on each node composing the file system cluster.

\subsection{TýrFS implementation}

In this section we detail the implementation of the above principles in a prototype file system optimized for small files, which we name TýrFS. We implement it as a thin layer atop Týr. The system is composed of a data service described in Section 12.3.1. directly mapped to Týr native semantics. A lightweight metadata management module runs on each server node. We describe in detail its implementation in Section 12.3.2. Finally, a client library exposes both a mountable and a native interface optimized for TýrFS. We detail it in Section 12.3.2.1

\subsubsection{Implementation of the data service}

The data service directly leverages the capabilities natively offered by Týr to efficiently distribute and store data in the cluster. A file in TýrFS is directly mapped to a blob in Týr, with the blob key being the absolute file path.

The predictable distribution of the data across the cluster that we describe in Section 12.2 .2 is inherited from Týr. The stored data can thus be located and accessed by 
TýrFS clients directly. Read and write operations as per the POSIX-I/O standard are mapped directly to the blob read and write operations offered by Týr.

Folders are also stored as a blob holding a list of all objects contained in it. As such, a folder list operation only requires reading the blob, while adding a file to a folder is implemented as a blob append. Although not implemented in our prototype, a background process running on each node would be responsible for compacting the file list when objects are deleted from a folder.

Týr provides data chunking for large files. In contrast to other distributed file systems such as Ceph, its transactional nature guarantees the correctness of read write operations spanning multiple chunks, consequently strictly sticking to the semantics guaranteed by the POSIX-I/O standard.

A side effect of using consistent hashing for data distribution is the need to move the physical location of the data when a file is moved in the folder hierarchy. This operation is implemented by writing "staple" records at the new location of the object and of its descendents, referencing their previous location. The data transfer occurs in the background. Moving a folder containing many descendents is consequently a costly operation. We argue that this trade-off is justified by the scarcity of such operations compared to the shorter critical path and thus higher performance of file reads and writes.

\subsubsection{Popularity-based metadata service}

The metadata service is implemented as a lightweight plugin running inside each Týr server process. Its responsibilities are threefold. First, it serves as a persistence layer for the files whose first chunk of data is stored on this process. Second, it integrates a dynamic replication agent, which analyzes the received requests to determine for which folders to replicate and store the metadata information locally. Finally, it serves as a resolver agent for the metadata requests, dispatching the requested information directly if available locally, or forwarding the request to the appropriate node determined using consistent hashing if not. The most interesting part of this metadata service is arguably the dynamic replication agent, which is the focus of this section.

Popularity as a dynamic replication metric: We use popularity as the main metric for dynamic replication to determine which folders to locally replicate metadata for. We 
define popularity as the number of metadata requests for any given folder in a defined time period. These popularity measurements are performed independently for each node. Consequently, on any given node, the more frequently metadata for a specific folder is requested the more likely it is to be considered for dynamic replication to that node. This metric is particularly suited for workloads accessing vast numbers of small files. Considering that at least a metadata request is needed for reading each file, a data set composed of many files will be significantly more likely to have the metadata for its ancestor folders replicated locally than a data set composed of a single large file.

To measure the popularity for each folder, we record the number of times its metadata is requested to the local resolver agent. Keeping exact counters for each possible folder is memory-intensive, so we only focus on the $k$ most frequently requested folders, $k$ being user-configurable. This problem is known as top- $k$ counting. A variety of memory-efficient, approximate solutions to this problem exist in the literature. For this use-case, such an approximate algorithm is tolerable as popularity estimation errors cannot jeopardize the correctness of the system. Our implementation uses SpaceSaving (120) as top- $k$ estimator. It guarantees strict error bounds for approximate request counts, while exhibiting a small, predictable memory complexity of $O(k)$. The output of the algorithm is an approximate list of the $k$ folders for which the metadata is the most frequently requested since the data structure has first been initialized. We perform these measurements over a sliding time window in order to favor immediate popularity over historical measurements.

When should an object metadata be dynamically replicated: Whenever a metadata request arrives at the metadata resolver for a folder whose metadata is not locally replicated, the resolver agent determines whether or not it should be replicated. It does so by adding the requested folder path in the current time window for the top- $k$ estimator. If the folder path is present in the output of the estimator, this path should be considered as popular and the associated metadata should be replicated locally. The agent determines which node holds the metadata for this folder, and remotely fetches the metadata from this node. Should the metadata be kept locally, it is stored in a temporary local store. In any case, the fetched metadata is returned to the client. This process is highlighted by the pseudo-code of Algorithm 1 . 


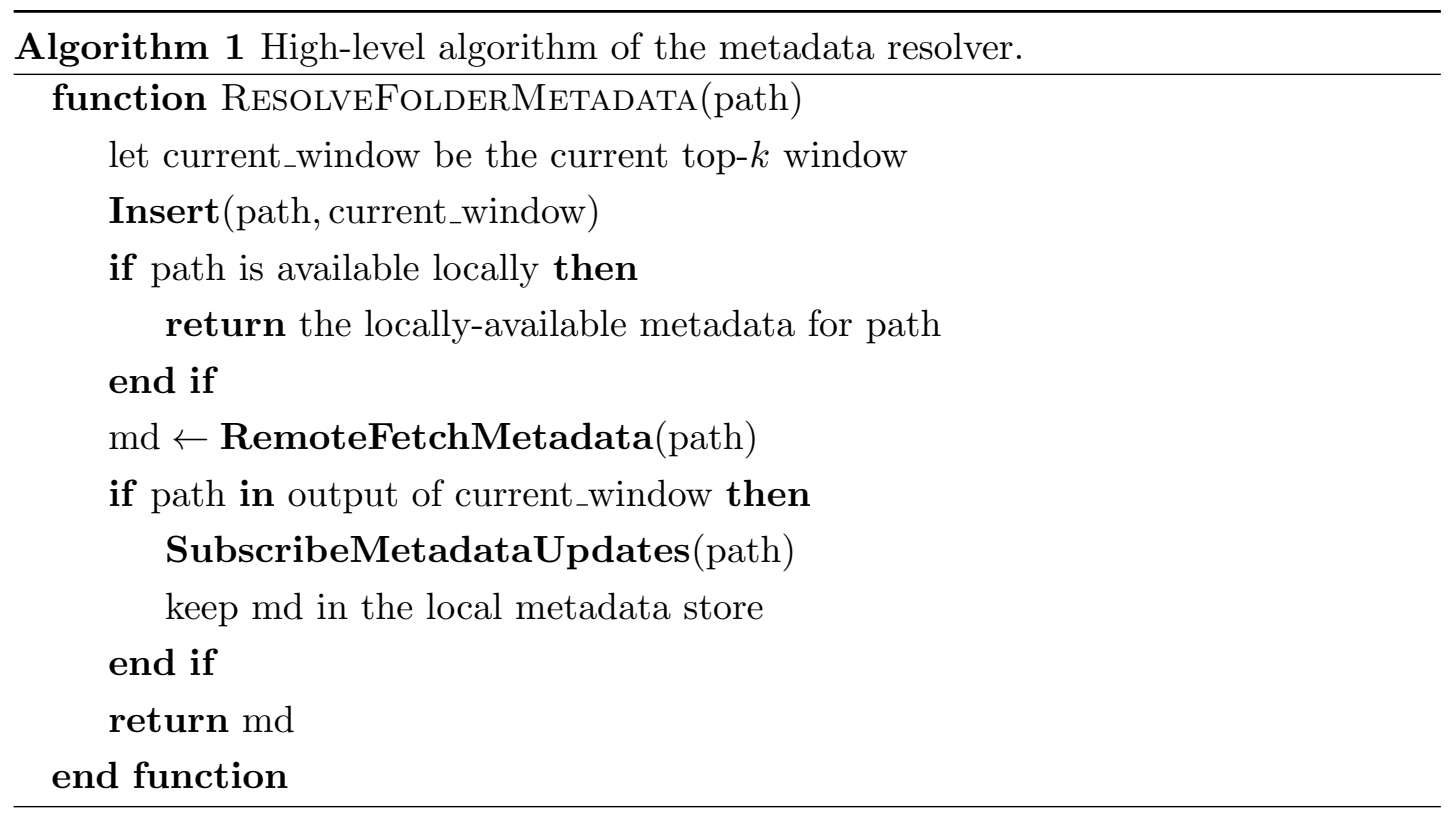

Handling move operations: Although folder permission modifications are a very rare occurrence in real-world applications, such metadata updates should nonetheless be consistently forwarded to metadata replicas. We integrate inside the TýrFS metadata agent a synchronous metadata notification service. When the permissions for any given folder are modified, this service synchronously notifies all nodes locally storing a dynamic replica of this metadata. Subscription to updates is performed atomically by a remote metadata agent during the metadata fetch if the remote agent has determined that this metadata should be dynamically replicated to it. Acknowledgement is sent to the client requesting the metadata change only after the update has been applied to all dynamic replicas.

A background bookkeeping service monitors changes in the current time window for the top- $k$ estimator in order to identify the metadata for previously-popular objects that are not popular anymore. The locally available metadata is marked for deletion. It will be kept locally as long as the locally available storage permits. When space needs to be reclaimed, the node deletes the locally-replicated metadata marked for deletion. It also synchronously cancels its subscription for metadata update notifications. 


\subsubsection{TýrFS client}

The TýrFS client provides two different interfaces. A native interface exposes all POSIX-I/O functions. In addition, a mountable interface, itself based on the native library, allows TýrFS to be mounted as any other file system. The mountable interface leverages the FUSE library. The native interface is implemented as a thin wrapper around the Týr native client library, which handles all the message serialization and communication with the storage cluster.

As an optimization to the TýrFS design, the native interface adds a function to those of the POSIX-I/O standard. In common file systems, reading a file is split between the metadata fetching (open) and the data read operation (read). Within the design of TýrFS embracing collocation of data and metadata, this can be further optimized by combining the two operations into a single one. Doing so saves the cost of an additional client request to the file system cluster, therefore reducing read latency and increasing throughput. The TýrFS client proposes a native method exposing such behavior, named fetch, which opens and reads a file given by its absolute path.

\subsection{Experimental setup}

We execute all following experiments on the Grid'5000 testbed as in Section 12.1. The machines we use are part of the Paravance cluster (9), located in Rennes. Each node embeds 16 cores ( $2 \times$ 8-core Intel Xeon E5-2630v3 processor) as well as 128 GB RAM. Connectivity between nodes is done using low-latency 10 Gigabit Ethernet. All nodes are connected to the same switch. Node-local storage is provided by two $600 \mathrm{~GB}$ spinning drives.

We compare the performance of TýrFS with that of multiple file systems, from both the HPC and the Big Data communities. Lustre 2.10.0 (142) and OrangeFS 2.9.6 (142) are deployed according to best practices. With Lustre we dedicate one node for metadata. For Big Data applications we compare TýrFS with Ceph 12.2.0 (167) and HDFS 2.8.0 (31).

We benchmark the performance of TýrFS using two different application profiles. For HPC file systems we use a simple benchmark. This MPI program opens a file, and performs append operations to it from a configurable number of ranks. Each rank performs appends sequentially. It accesses the file system through the TýrFS native client 
and leverages the fetch operation it provides. The benchmark reads through the whole pre-loaded random dataset from all ranks, evenly distributing the last across all ranks. No actual computation is performed on the data. Big Data Analytics applications are running atop Spark 2.2.0 (173) and are extracted from SparkBench (103).

For Lustre and OrangeFS we use 24 machines to deploy the storage systems, plus 8 machines to deploy the client applications. With Lustre we dedicate one node for metadata management. Big Data applications are deployed alongside storage on the whole 48 nodes of the cluster.

\subsection{Performance in support of HPC applications}

We first compare the performance results obtained with TýrFS to those of Lustre and OrangeFS. The curves for Lustre and GPFS are reproduced for reference. The size of the input data set is constant. We vary the number of input files between $3,840 \times 32$ MB files ( 1 file / rank) to $3.84 \times 10^{6} \times 32 \mathrm{~KB}$ files (10.000 files / rank). The data is generated randomly.

In Figure 12.6 we plot the total execution time of the benchmark for TýrFS, Lustre and OrangeFS. To evaluate the baseline performance of the metadata replication system, we also plot the performance achieved by TýrFS with dynamic replication disabled. The performance for large files is on par with that of Lustre or OrangeFS due to the possible amortization of the metadata requests. However, TýrFS shows a significant performance improvement over those two systems for small files. Disabling metadata replication causes TýrFS performance to drop at the level of other distributed file systems. The similarity of the performance of TýrFS without metadata replication with that of OrangeFS is due to the fact that both embrace akin distributed metadata management principles. This unequivocally demonstrates the effectiveness of dynamic metadata replication with small files, enabling the file system to offer high read performance even at the lowest file sizes.

Figure 12.7 shows the relative breakdown of the I/O time between the metadata (M) and data (D) access inside the fetch operation. We note that the relative time spent on metadata access as the data file increases tends to decrease with TýrFS. This clearly contrasts with other file systems, in which the throughput drop is mainly due to the high cost of metadata management. In TýrFS, the main factor impacting 


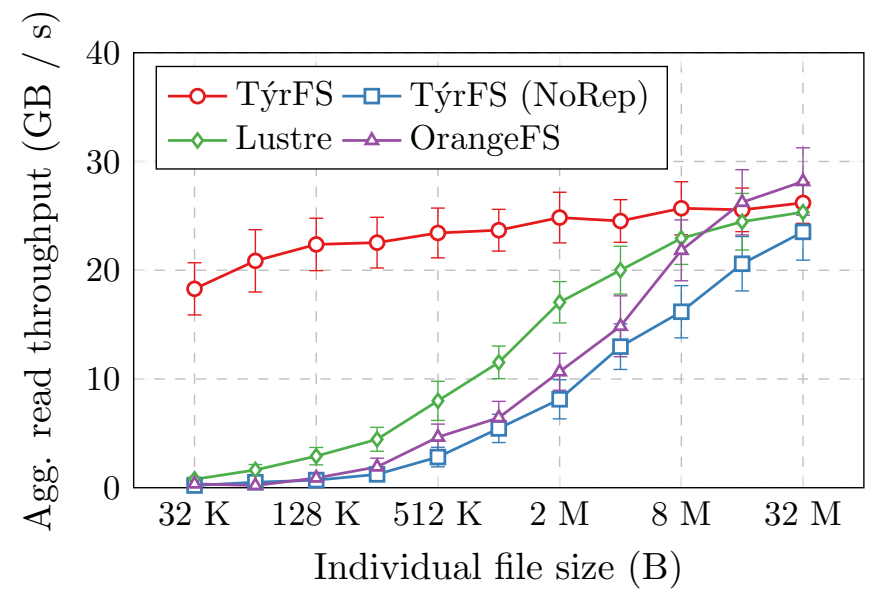

Figure 12.6: TýrFS, Lustre and OrangeFS read throughput for a fixed-size data set, varying the size of the individual files it is composed of.

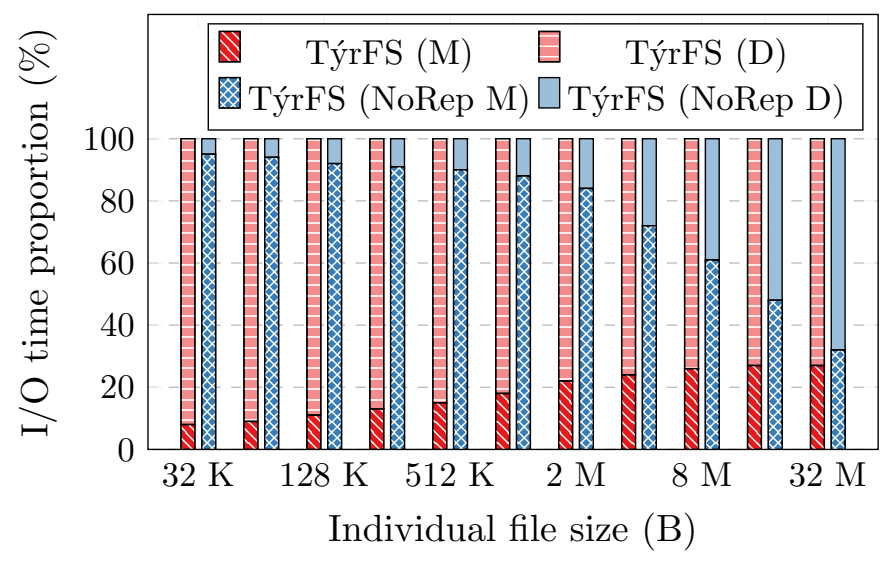

Figure 12.7: Breakdown of reads between metadata (M) and data (D) access for a fixed-size data set varying the size of the individual files it is composed of.

read time is the added cost of issuing additional read requests. The efficiency of the dynamic metadata replication is highlighted by the performance of TýrFS with this service disabled, which here also is similar to that of other file systems.

\subsubsection{Scalability}

Týr, the base building block of TýrFS, has a demonstrated near-linear horizontal scalability that makes it particularly suitable for large-scale use-cases. TýrFS does not introduce any centralization that could jeopardize these features. In particular, it inherits from OrangeFS the distributed metadata management principles, which support the horizontal scalability of this system. As such, we argue that TýrFS should be able to inherit this desirable feature. In this section we formally demonstrate the horizontal scalability of TýrFS. 


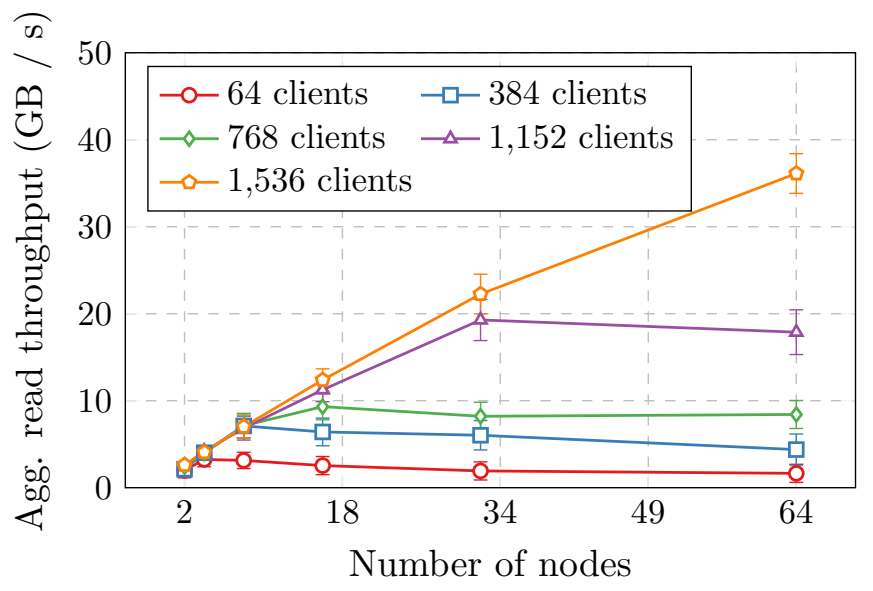

Figure 12.8: TýrFS horizontal scalability, varying the number of clients and the number of cluster nodes with $32 \mathrm{~KB}$ input files.

We adopt the same setup as in the previous section. We vary the number of storage nodes from 2 to 64 on the Paravance cluster. We setup clients on up to 24 nodes of a second Grid'5000 cluster: Parasilo. Both clusters are co-located in the same facility, and are interconnected with low-latency $2 \times 40 \mathrm{G}$ Ethernet links aggregated using the Link Aggregation Control Protocol (LACP). Each client node runs 64 ranks of the MPI read benchmark that concurrently reads files from our random, pre-loaded 1.22 TB data set. We run the benchmark for 5 minutes, reading the dataset from within an endless loop. The dataset for this experiment contains $3.84 \times 10^{7} \times 32 \mathrm{~KB}$ files.

In Figure 12.8 we plot the measured aggregated throughput of TýrFS across all clients, varying the number of nodes from 2 to 64 and the number of concurrent clients from 64 to 1,536. The results demonstrate that TýrFS shows a near-linear horizontal scalability when adding nodes to the storage cluster, being able to serve more clients with higher aggregate throughput. The decrease in throughput observed with the smallest cluster sizes when increasing the number of clients is due to the saturation of the storage servers. In this configuration, and despite working on a data set composed of very small files, we measured the aggregated throughput of TýrFS as high as 36 GB / s across all clients with 64 storage servers and 1,536 concurrent client ranks.

\subsection{Performance in support of BDA applications}

For these experiments we setup a configuration according to the best practices for BDA. We deploy both the storage system and the computation on all 48 nodes of the cluster. 


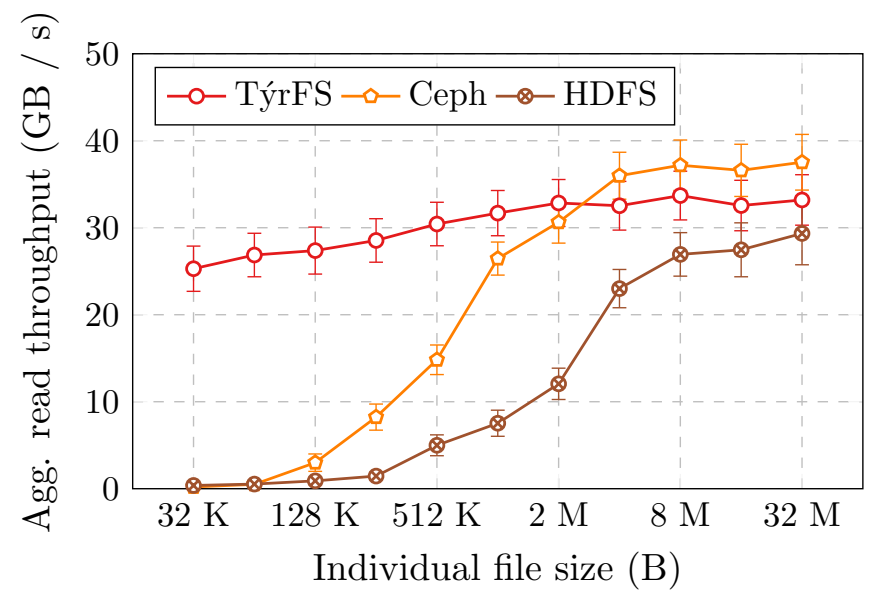

Figure 12.9: TýrFS, Ceph and HDFS read throughput for a fixed-size data set, varying the size of the individual files it is composed of.

We compare the performance of Týr with that of Ceph and HDFS. The experiments are based on a series of different Spark applications.

\subsubsection{Measuring read throughput}

We leverage SparkBench (103) to confirm that the design of TýrFS yields significant performance improvements with small files for applications showing a wide range of I/O profiles. We use exactly the same setup as in the previous experiment, and execute three read-intensive benchmarks extracted from the benchmark suite: Connected Component (CC), Grep (GR) and Decision Tree (DT). These benchmarks are intended to evaluate the I/O performance of TýrFS when faced with different workloads types, respectively graph processing, text processing and machine learning.

In Figure 12.9 we plot the aggregate throughput obtained across all nodes when varying the size of the input data set files. Overall, the results are very similar to those obtained with Lustre and OrangeFS. This is not surprising, and is mostly due to the very similar architecture of all these file systems, in which the relative cost of metadata management increases as the file size decreases. For these experiments as well, the throughput drop we observe with TýrFS when using $32 \mathrm{~KB}$ files instead of 32 MB files is only of about $25 \%$. In similar conditions, other systems show a much more significant throughput drop of more than $90 \%$. This results in a $3.8 \times$ increase of total computation time with OrangeFS, wasting computing resources. 


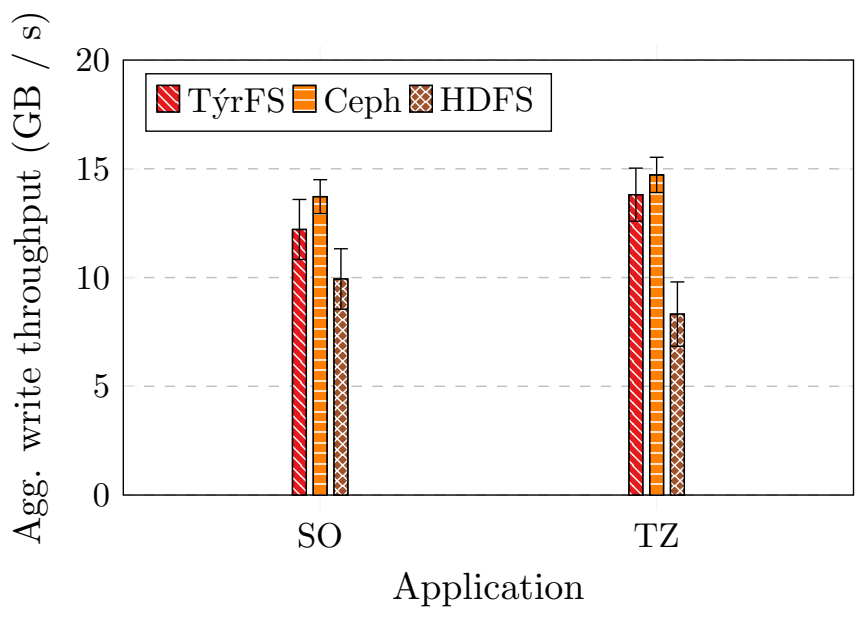

Figure 12.10: TýrFS, Ceph and HDFS write throughput with Sort (balanced I/O) and Tokenizer (write-intensive) applications.

\subsubsection{Measuring write throughput}

Not all applications are read-intensive. Although write performance is not a main target of TýrFS, it is critical to understand precisely the impact of dynamic metadata replication for balanced and write-intensive workloads as well. To do so, we add two more applications to the experiment: Sort (SO) from SparkBench, which illustrates a balanced I/O workload, and Tokenizer (TZ), which provides a write-intensive one. These applications are the same as in Chapters 5 and 11 .

In Figure 12.10 we plot the measured write throughput for Sort and Tokenizer with TýrFS, Ceph and HDFS. The performance of the write operations are similar across the two experiments. TýrFS exhibits a write performance consistent with that of Ceph. Ceph outperforms both TýrFS and HDFS for write throughput by a margin of respectively 8 and $35 \%$.

\subsubsection{Application completion time}

Considering the application as a whole, Figure 12.11 summarizes our results for Big Data applications. We measure the application completion time difference for $32 \mathrm{MB}$ files relative to that obtained with $32 \mathrm{~KB}$ files. The throughput advantage of TýrFS over Ceph and HDFS with small files is clearly visible on the application completion time, allowing it to retain at least $73 \%$ of its performance when using $32 \mathrm{~KB}$ instead of $32 \mathrm{MB}$ files. Ceph and HDFS in similar conditions retain less than $5 \%$ of their performance, hence wasting costly resources. Overall, the impact of small input files is 


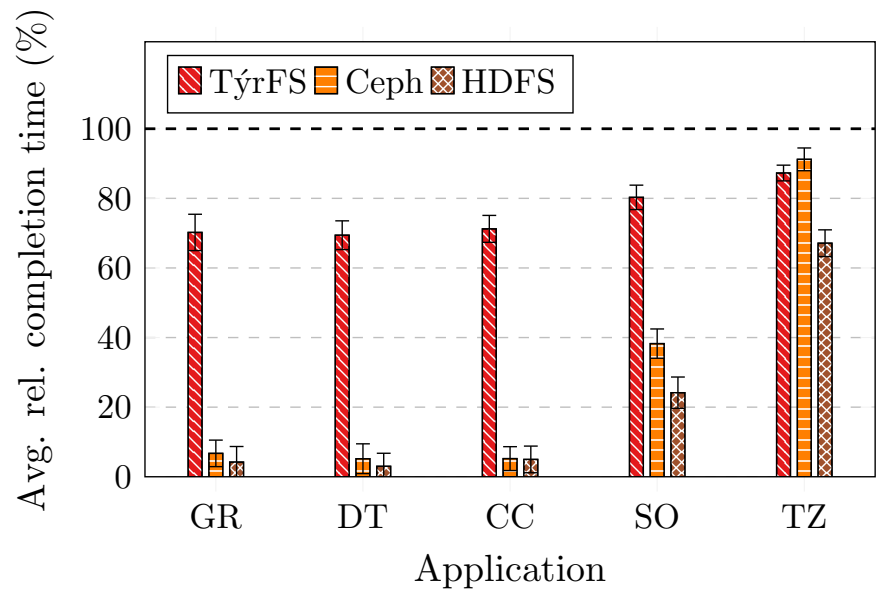

Figure 12.11: Application completion time difference with $32 \mathrm{MB}$ input files relative to that obtained with 32 KB input files, with TýrFS, Ceph and HDFS. 100\% represents the baseline performance for $32 \mathrm{MB}$ files.

significantly lower for all three systems with Sort and Tokenizer applications because of the higher influence on writes, for which all three systems show comparable performance. The write-intensive Tokenizer application gives a $4 \%$ advantage to Ceph because of its slightly higher write throughput compared to TýrFS.

\subsection{Conclusions}

We propose TýrFS as a POSIX I/O compliant file system atop Týr, especially optimized for small files. TýrFS enables clients to locate any piece of data in the cluster independently of any metadata server. A dynamic metadata replication module adapts to the workload to efficiently replicate the necessary metadata on nodes from which the associated data is frequently accessed. Clients can then read frequently accessed data directly, without involving additional servers. We did demonstrate a performance improvement of more than an order of magnitude with smaller file sizes compared to state-of-the-art storage systems.

TýrFS is specifically designed to benefit use cases involving large numbers of small files. Yet, while not the scope of this thesis, no fundamental part of its design make TýrFS unfit for large files. The metadata distribution in TýrFS will however be less effective with large files. The main reason is that the metadata for any given file is co-located only with the first chunk of the file. Reading a different chunk will trigger a remote metadata fetch for the file, which is unnecessary for small files.

These results further demonstrate the effectiveness of Týr design and principles in 
the context of storage-based convergence between HPC and BDA. In particular, TýrFS supports the needs of legacy applications expecting full POSIX-IO compliance, while enabling applications built to leverage blobs to benefit from additional performance improvements compared to traditional file-based storage. 


\section{Part IV}

\section{Conclusions: Achievements and Perspectives}





\section{Chapter 13}

\section{Conclusions}

The challenges faced for achieving convergence between HPC and BDA platforms are staggering. While the literature tackling these hard problems for data processing is rich, very few works have studied this convergence under the angle of storage. The storage stacks leveraged by HPC and BDA applications remain largely separated today, effectively preventing application portability between platforms and limiting their applicability to a new generation of large scale data processing and analytics platforms.

Furthermore, the weaknesses of existing approaches limit their applicability in the context of HPC and BDA convergence. In particular, the centralized metadata management associated with the legacy file-based storage model causes high read and write latency because of the metadata management overhead. Existing blob storage systems either lack low-latency direct read capability, which hinders read throughput, or use lock-based concurrency control which diminishes performance when facing high write concurrency. Finally, the need for write coordination is left up to the user to manage, making efficient applications very difficult to design when such features are needed.

The work presented in this thesis led to the design of Týr, a storage system that addresses many of the limitations listed above. We experimentally proved the usefulness of Týr's design in a variety of large-scale applicative contexts on both HPC and BDA platforms, showing its applicability in the context of storage-based convergence.

The main objective set at the beginning of this manuscript is thus fulfilled. In the rest of this chapter, we underline the relevance of our contributions presented so far, and show how they enabled us to solve a series of sub-objectives centered on our 
main goal. Finally, we discuss several choices we made and detail related research perspectives that are yet to be explored.

\subsection{Achievements}

Blobs as a storage model for HPC and BDA convergence. By carefully studying the state of the practice in the field of large-scale storage for HPC and BDA platforms, we proposed binary large objects (blobs) as a relevant storage model for convergence. Indeed, the semantics and access patterns they permit are close to those of file systems, hence enabling transparent support for legacy applications. By not requiring centralized metadata management, they permit a variety of protocol optimizations such as direct reads, supporting their high throughput but coming at the expense of write consistency guarantees. Finally, state-of-the-art approaches demonstrated the benefits of efficient multi-version concurrency control for applications exhibiting high write contention. Yet, providing all these benefits at the same time is challenging. We consequently noted the limitations of existing approaches to that extent, either providing low storage operation latency or high performance under concurrent access patterns, but not both.

A set of key design principles for large-scale, efficient converging storage. We proposed a set of key design principles that enable building a storage system targeted at HPC and BDA convergence by overcoming most limitations of the existing approaches. First, we advocate the relevance of predictable data and metadata distribution using consistent hashing techniques to enable direct read and write access to data without requiring centralized metadata management, while also uniformly distributing the load across the cluster. Transparent multi-version concurrency control supports high write performance for highly-contended I/O patterns that are increasingly common for a variety of applications (i.e. append logs, data aggregation). These features are leveraged to provide ACID transactional semantics to ensure data consistency while letting users orchestrate multiple storage calls without requiring complex application-level coordination such as locking. Finally, these semantics enable atomic transform operations which bring significant throughput increase to applications performing data aggregation by reducing the amount of communication required for simple update patterns. 
Týr: applying these principles into practice. We designed Týr, a distributed blob storage system implementing all of the above design principles. We define the protocols that enable Týr to deliver its promise of strong concurrency, high velocity and horizontal scalability. A family of read protocols either provide direct access to the data, consistent multi-chunk read capability or transactional read isolation. While all these protocols provide strong read consistency, Týr is able to transparently select the protocol providing the highest throughput and lowest latency in a specific request context. A lightweight transactional write protocol coordinates writes across the system, providing linearizability at scale as well as supporting high-velocity atomic transform operations. This base protocol is extended with efficient version bookkeeping that supports the background garbage collection of expired data while retaining the repeatability of transactional reads. We fully implemented all these principles and protocols in a software prototype.

Real-life benefits demonstrated through multiple applicative scenarios We successfully proved the relevance of Týr's design in four different applicative scenarios:

\section{- A storage backend for a BDA monitoring system that substantially} improves data aggregation performance. In the context of large-scale data monitoring, the efficiency of data aggregation is key to deliver relevant information in near real-time. We developed a Týr-based storage backend for a real-life monitoring system from CERN, and evaluated its performance using production data. Týr demonstrated superior performance for data aggregation compared to other systems thanks to its transactional semantics atomic transform operations while constantly providing higher consistency guarantees and solid read performance due to the predictable data distribution it leverages. This work is the result of a collaboration with María S. Pérez, Alexandru Costan, Gabriel Antoniu and Jesús Montes.

- A storage platform for computational steering that supports highvelocity data logging at very large scale. We have successfully deployed Týr in the context of data logging and computational steering at very large scale on HPC platforms. Through a series of synthetic experiments, we proved Týr's superior performance under extreme access concurrency. It provides up to $30 \%$ 
higher append performance compared to other storage systems and up to 5 orders of magnitude compared to state-of-the-art distributed file systems. We proved the horizontal scalability of Týr in this context by deploying our solution on up to 120,000 cores of the Theta supercomputer. This work was carried out during a 3-month internship at Argonne National Laboratory under the supervision of Philip Carns and Robert Ross, as well as María S. Pérez, Alexandru Costan, and Gabriel Antoniu.

- A versatile storage system for a variety of HPC and BDA applications. We demonstrated the applicability of Týr to provide storage for legacy applications through a series of thorough experiments with data-intensive HPC and BDA applications. In particular, we proved that the combination of its multi-version concurrency control and direct read access capability constantly provides highperformance access to the data despite the variety of I/O patterns exhibited. We could successfully replicate the results obtained for HPC applications at scale on the Theta supercomputer. This work is the result of a collaboration with Álvaro Brandon, Yevhen Alvorov, María S. Pérez, Alexandru Costan, Gabriel Antoniu, Michael Kuhn, Philip Carns and Thomas Ludwig.

\section{- TýrFS, a Týr-based distributed file system that improves performance} with many small files. We finally demonstrated how Týr design can serve as a base building block for other storage abstractions, taking the example of a file system optimized for the hard use-case of many small files. Indeed, highly-efficient direct read capabilities support the very low read overhead that is required to efficiently cope with such difficult I/O pattern. We combined this feature with dynamic metadata replication, showing performance improvements of more than an order of magnitude compared to other file systems from both HPC and BDA communities. This work was carried out in collaboration with María S. Pérez, Alexandru Costan and Gabriel Antoniu.

\subsection{Perspectives}

The choices we made during the development of this work favored some research lines over others. Although this thesis yielded a wide variety of achievements, there certainly 
are many directions that were left unexplored. In this section we detail the potential research areas that address new challenges related to this work.

Leveraging transactional operations in HPC contexts. In this thesis we did demonstrate how the semantics and the set of guarantees offered by Týr's transactional architecture could prove beneficial for HPC applications in distributed logging contexts. Also, we did detail in TýrFS how transactions could prove crucial for the design of high-level storage abstractions. Indeed, series of potential use-cases exist for explicit storage coordination on HPC systems. We are convinced that transactions have a case in HPC applications for collaboratively maintaining complex data structures. However, the lack of such semantics on current platforms makes it very hard to formally demonstrate the effectiveness of transactions when leveraged directly at the application level. Thus, a promising research direction is to study the impact of transactional operations and optimistic write coordination in the design of large-scale applications for next-generation HPC platforms. Such progress would obviate the need for application-level write coordination in most cases while at the same time greatly simplifying the design of several I/O framework operations, among which MPI Collective $\mathrm{I} / \mathrm{O}$.

Exploring a wider variety of transactional storage abstractions over transactional blobs. We have shown with TýrFS how transactional blobs have the capacity to serve as low-level distributed storage layer for a variety of storage abstractions. Yet, besides distributed file systems, there are many such abstractions that could potentially leverage blob storage. These include transactional key-value stores, transactional wide column databases or graph databases. A great deal of work certainly exists in the domain, but we believe the angle of convergence is yet to be considered. Indeed, such abstractions could be deployed in a wide variety of applicative contexts, from HPC and BDA communities. One could imagine proposing Týr as a base storage layer on both cloud- and HPC-oriented platforms, letting the users choose the exact interface and semantics they need without investing time and effort on low-level data management or portability across platforms.

Geo-distributed environments. A challenge closely tied with the Big Data movement is the increasing need to geographically distribute data processing and warehous- 
ing. This is due to multiple factors, two of the most important ones being the lack of sufficient resource availability in a single location for very large data sets and the natural geo-distribution of the data sources associated with the high cost of transferring such large amounts of data over large distances. The challenges associated with such distribution are even higher when considering transactional semantics and strong consistency models, which potentially incur latency that is incompatible with the near real-time requirements of many data analytics applications. A promising future research question is how transactional blob storage can fit in this large picture, both by reducing the processing latency and proposing adequate consistency models and trade-offs in the angle of HPC and BDA convergence.

Týr as a base for HPC and BDA convergence. The future work associated with HPC and BDA convergence spans far beyond storage. We do believe that this movement will lead to many unexplored and unsolved research directions, which notably include inherently convergent wide-area, multistage workflows. One could imagine HPC-like programming tools and techniques to fit in the picture, or further cloud processing systems to be adapted to the specifics of HPC platforms. We advocate that a convergent storage platform such as the one Týr provides is able to fuel portability and reduce the scope of the challenges of data processing, by proposing a single storage model for a wide variety of upcoming applications and use-cases designed from the ground up with convergence in mind. 


\section{Bibliography}

[1] Aurora. https://aurora.alcf.anl.gov, 2014. Accessed: 2017-11-20. 103, 107

[2] Marenostrum. https://www.bsc.es/marenostrum/marenostrum, 2014. Accessed: 2017-11-20. 103

[3] Mira. https://www.alcf.anl.gov/mira, 2014. Accessed: 2017-11-20. 106

[4] Sierra. https://computation.llnl.gov/computers/sierra, 2014. Accessed: 2017-11-20. 103

[5] Summit. https://www.olcf.ornl.gov/olcf-resources/compute-systems/ summit/, 2014. Accessed: 2017-11-20. 103

[6] Big Data and Extreme-scale Computing (BDEC). http://www.exascale.org/ bdec/, 2016. Accessed: 2017-11-24. 46

[7] Amazon Neptune. https://aws.amazon.com/neptune/, 2017. Accessed: 201801-07. 26

[8] FlatBuffers. http://google.github.io/flatbuffers/, 2017. Accessed: 201711-27. 88

[9] Grid'5000 - Rennes hardware. https://www.grid5000.fr/mediawiki/index. php/Rennes:Hardware, 2017. Accessed: 2017-10-14. 134

[10] KCP. https://github.com/skywind3000/kcp, 2017. Accessed: 2017-11-28. 88

[11] Running Spark on YARN. https://spark.apache.org/docs/latest/ running-on-yarn.html, 2017. Accessed: 2017-11-27. 51 
[12] Zlog.

https://nwat.xyz/blog/2014/10/26/ zlog-a-distributed-shared-log-on-ceph/, 2017. Accessed: 2017-11-28. 106

[13] Ceph: differences from POSIX. http://docs.ceph.com/docs/kraken/cephfs/ posix/, 2018. Accessed: 2018-01-07. 28, 31, 123

[14] Aamodt, K., Quintana, A. A., Achenbach, R., Acounis, S., Adamová, D., Adler, C., Aggarwal, M., Agnese, F., Rinella, G. A., Ahammed, Z., ET AL. The ALICE experiment at the CERN LHC. Journal of Instrumentation 3, 08 (2008), S08002. 5, 93,94

[15] Abramovici, A., Althouse, W. E., et Al. LigO: The laser interferometer gravitational-wave observatory. science (1992), 325-333. 124

[16] Agrawal, B., Chakravorty, A., Rong, C., and Wlodarczyk, T. W. R2time: a framework to analyse open TSDB time-series data in HBase. In Cloud Computing Technology and Science (CloudCom), 2014 IEEE 6th International Conference on (2014), IEEE, pp. 970-975. 16

[17] Agrawal, D., Butt, A., Doshi, K., Larriba-Pey, J.-L., Li, M., Reiss, F. R., RaAb, F., Schiefer, B., Suzumura, T., And Xia, Y. SparkBencha spark performance testing suite. In Technology Conference on Performance Evaluation and Benchmarking (2015), Springer, pp. 26-44. 47

[18] Akioka, S., And Muraoka, Y. Hpc benchmarks on amazon ec2. In Advanced Information Networking and Applications Workshops (WAINA), 2010 IEEE 24th International Conference on (2010), IEEE, pp. 1029-1034. 2

[19] Ali, N., Carns, P., Iskra, K., Kimpe, D., Lang, S., Latham, R., Ross, R., Ward, L., and Sadayappan, P. Scalable I/O forwarding framework for high-performance computing systems. In Cluster Computing and Workshops, 2009. CLUSTER'09. IEEE International Conference on (2009), IEEE, pp. 1-10. 21

[20] Amazon. Amazon web services. https://aws.amazon.com/, 2015. Accessed: 2017-12-10. 16, 24, 27 
[21] Anderson, J. C., Lehnardt, J., and Slater, N. CouchDB: The Definitive Guide: Time to Relax. " O'Reilly Media, Inc.", 2010. 25

[22] Bailis, P., Fekete, A., Franklin, M. J., Ghodsi, A., Hellerstein, J. M., And Stoica, I. Coordination avoidance in database systems. Proceedings of the VLDB Endowment 8, 3 (2014), 185-196. 68

[23] Bairavasundaram, L. N., Arpaci-Dusseau, A. C., Arpaci-Dusseau, R. H., Goodson, G. R., And Schroeder, B. An analysis of data corruption in the storage stack. ACM Transactions on Storage (TOS) 4, 3 (2008), 8. 31

[24] Balakrishnan, M., Malkhi, D., Prabhakaran, V., Wobber, T., Wei, M., AND Davis, J. D. Corfu: A shared log design for flash clusters. In Proceedings of the 9th USENIX conference on Networked Systems Design and Implementation (2012), USENIX Association, pp. 1-1. 106

[25] Balouek, D., Carpen Amarie, A., Charrier, G., Desprez, F., Jeannot, E., Jeanvoine, E., Lèbre, A., Margery, D., Niclausse, N., Nussbaum, L., Richard, O., Pérez, C., Quesnel, F., Rohr, C., and Sarzyniec, L. Adding virtualization capabilities to the Grid'5000 testbed. In Cloud Computing and Services Science, I. Ivanov, M. Sinderen, F. Leymann, and T. Shan, Eds., vol. 367 of Communications in Computer and Information Science. Springer International Publishing, 2013, pp. 3-20. 49

[26] Bernstein, P. A., And Goodman, N. Multiversion concurrency control-theory and algorithms. ACM Transactions on Database Systems (TODS) 8, 4 (1983), 465-483. 28

[27] Berriman, G., Good, J., Curkendall, D., et Al. Montage: An on-demand image mosaic service for the nvo. In Astronomical Data Analysis Software and Systems XII (2003), vol. 295, p. 343. 124

[28] Bloom, B. H. Space/time trade-offs in hash coding with allowable errors. Communications of the ACM 13, 7 (1970), 422-426. 81 
[29] Boichat, R., Dutta, P., Frølund, S., and Guerraoui, R. Deconstructing paxos. ACM Sigact News 34, 1 (2003), 47-67. 39

[30] Bolze, R., Cappello, F., Caron, E., Daydé, M., Desprez, F., Jeannot, E., Jégou, Y., Lanteri, S., Leduc, J., Melab, N., et Al. Grid'5000: A large scale and highly reconfigurable experimental grid testbed. The International Journal of High Performance Computing Applications 20, 4 (2006), 481-494. 124

[31] Borthakur, D., et Al. Hdfs architecture guide. Hadoop Apache Project 53 (2008). 134

[32] Branm, P. J., And Others. The Lustre storage architecture. White Paper, Cluster File Systems, Inc., Oct 23 (2003). 2

[33] BRAY, T. The javascript object notation (JSON) data interchange format. 24

[34] Bray, T., Paoli, J., Sperberg-Mcqueen, C. M., Maler, E., And Yergeau, F. Extensible markup language (XML). World Wide Web Journal 2, 4 (1997), 27-66. 24

[35] Brewer, E. A. Towards robust distributed systems. In PODC (2000), vol. 7. 40

[36] Bryan, G. H., And Fritsch, J. M. A benchmark simulation for moist nonhydrostatic numerical models. Monthly Weather Review 130, 12 (2002), 2917-2928. 124

[37] Butnaru, D., Buse, G., And Pflüger, D. A parallel and distributed surrogate model implementation for computational steering. In 2012 11th International Symposium on Parallel and Distributed Computing (June 2012), pp. 203-210. 104

[38] Byun, C., Arcand, W., Bestor, D., Bergeron, B., Hubbell, M., Kepner, J., McCabe, A., Michaleas, P., Mullen, J., O’Gwynn, D., et Al. Driving big data with big compute. In High Performance Extreme Computing (HPEC), 2012 IEEE Conference on (2012), IEEE, pp. 1-6. 25

[39] Calder, B., Wang, J., Ogus, A., Nilakantan, N., Skjolsvold, A., McKelvie, S., Xu, Y., Srivastav, S., Wu, J., Simitci, H., et al. Windows azure 
storage: a highly available cloud storage service with strong consistency. In Proceedings of the Twenty-Third ACM Symposium on Operating Systems Principles (2011), ACM, pp. 143-157. 27, 95

[40] Cao, M., Bhattacharya, S., and Ts'o, T. Ext4: The next generation of ext2/3 filesystem. In $L S F$ (2007). 129

[41] Carbone, P., Katsifodimos, A., Ewen, S., Markl, V., Haridi, S., And Tzoumas, K. Apache flink: Stream and batch processing in a single engine. Bulletin of the IEEE Computer Society Technical Committee on Data Engineering 36, 4 (2015). 46

[42] Carlson, J. L. Redis in action. Manning Publications Co., 2013. 96

[43] Carns, P., Harms, K., Kimpe, D., Ross, R., Wozniak, J., Ward, L., Curry, M., Klundt, R., Danielson, G., Karakoyunlu, C., et al. A case for optimistic coordination in hpc storage systems. In High Performance Computing, Networking, Storage and Analysis (SCC), 2012 SC Companion: (2012), IEEE, pp. 48-53. 68, 69

[44] Chang, F., Dean, J., Ghemawat, S., Hsieh, W. C., Wallach, D. A., Burrows, M., Chandra, T., Fikes, A., And Gruber, R. E. Bigtable: A distributed storage system for structured data. ACM Transactions on Computer Systems (TOCS) 26, 2 (2008), 4. 25

[45] Chodorow, K. MongoDB: The Definitive Guide: Powerful and Scalable Data Storage. " O'Reilly Media, Inc.", 2013. 2, 25

[46] Collet, Y. Lz4: Extremely fast compression algorithm. 88

[47] Connolly, T. M., And BegG, C. E. Database systems: a practical approach to design, implementation, and management. Pearson Education, 2005. 38

[48] Cook, R. L., Porter, T., and Carpenter, L. Distributed ray tracing. In ACM SIGGRAPH computer graphics (1984), vol. 18, ACM, pp. 137-145. 47

[49] Copeland, M., Soh, J., Puca, A., Manning, M., and Gollob, D. Microsoft azure and cloud computing. In Microsoft Azure. Springer, 2015, pp. 3-26. 95 
[50] Corbett, J. C., Dean, J., Epstein, M., Fikes, A., Frost, C., Furman, J. J., Ghemawat, S., Gubarev, A., Heiser, C., Hochschild, P., et al. Spanner: Google's globally distributed database. ACM Transactions on Computer Systems (TOCS) 31, 3 (2013), 8. 33, 39, 41, 67

[51] Darling, A. E., Carey, L., And Feng, W. C. The design, implementation, and evaluation of mpiBLAST. Tech. rep., Los Alamos National Laboratory, 2003. 47

[52] Das, A., Gupta, I., and Motivala, A. Swim: Scalable weakly-consistent infection-style process group membership protocol. In Dependable Systems and Networks, 2002. DSN 2002. Proceedings. International Conference on (2002), IEEE, pp. 303-312. 83, 86

[53] Das, S., El Abbadi, A., And Agrawal, D. Elastras: An elastic transactional data store in the cloud. HotCloud 9 (2009), 131-142. 36

[54] De Prisco, R., Lampson, B., And Lynch, N. Revisiting the paxos algorithm. In International Workshop on Distributed Algorithms (1997), Springer, pp. 111125. 39

[55] DeCandia, G., Hastorun, D., Jampani, M., Kakulapati, G., Lakshman, A., Pilchin, A., Sivasubramanian, S., Vosshall, P., and Vogels, W. Dynamo: Amazon's highly available key-value store. ACM SIGOPS operating systems review 41, 6 (2007), 205-220. 2, 22, 33, 62, 63

[56] Dede, E., Sendir, B., Kuzlu, P., Hartog, J., and Govindaraju, M. An evaluation of cassandra for hadoop. In Cloud Computing (CLOUD), 2013 IEEE Sixth International Conference on (2013), IEEE, pp. 494-501. 46

[57] Dorier, M., Sisneros, R., Peterka, T., Antoniu, G., and Semeraro, D. Damaris/viz: a nonintrusive, adaptable and user-friendly in situ visualization framework. In Large-Scale Data Analysis and Visualization (LDAV), 2013 IEEE Symposium on (2013), IEEE, pp. 67-75. 104

[58] Dubois, M., Scheurich, C., And Briggs, F. Memory access buffering in multiprocessors. In ACM SIGARCH Computer Architecture News (1986), vol. 14, IEEE Computer Society Press, pp. 434-442. 32 
[59] Escriva, R., And Sirer, E. G. The design and implementation of the warp transactional filesystem. In 13th USENIX Symposium on Networked Systems Design and Implementation (NSDI 16) (2016), USENIX Association. 36, 61

[60] Escriva, R., Wong, B., And Sirer, E. G. Hyperdex: A distributed, searchable key-value store. In Proceedings of the ACM SIGCOMM 2012 conference on Applications, technologies, architectures, and protocols for computer communication (2012), ACM, pp. 25-36. 33, 41

[61] Escriva, R., Wong, B., And Sirer, E. G. Warp: Lightweight multi-key transactions for key-value stores. arXiv preprint arXiv:1509.07815 (2015). 36 , 39, 41, 67, 72

[62] Fischer, P., Kruse, J., Mullen, J., Tufo, H., Lottes, J., And KerkeMEIER, S. Nek5000 - open source spectral element CFD solver. Argonne National Laboratory, Mathematics and Computer Science Division, Argonne, IL, see https://nek5000.mcs.anl.gov/index.php/MainPage (2008). 2

[63] Fox, G., Qiu, J., Jha, S., Ekanayake, S., and Kamburugamuve, S. Big data, simulations and hpc convergence. In Big Data Benchmarking. Springer, 2015, pp. 3-17. 16

[64] Fox, G. C., Shantenu, J., Judy, Q., Saliya, E., And Andre, L. Towards a comprehensive set of big data benchmarks. Advances in Parallel Computing 26 (2015), 47-66. 46

[65] George, L. HBase: the definitive guide: random access to your planet-size data. " O'Reilly Media, Inc.", 2011. 25, 61

[66] Giardino, J., Haridas, J., And Calder, B. How to get most out of windows azure tables, 2013. 26

[67] Glendenning, L., Beschastnikh, I., Krishnamurthy, A., And Anderson, T. Scalable consistency in scatter. In Proceedings of the Twenty-Third ACM Symposium on Operating Systems Principles (2011), ACM, pp. 15-28. 67

[68] GlusterFS. The Gluster web site. https://www.gluster.org/, 2015. Accessed: 2018-01-04. 20 
[69] Graves, R., Jordan, T. H., Callaghan, S., Deelman, E., et al. CyberShake: A physics-based seismic hazard model for southern california. Pure and Applied Geophysics 168, 3-4 (2011), 367-381. 124

[70] Gray, J., And Reuter, A. Transaction processing: concepts and techniques. Elsevier, 1992. 2, 67

[71] Griffies, S. M. Elements of the modular ocean model (mom). GFDL Ocean Group Tech. Rep 7 (2012), 620. 47

[72] Gropp, W., And Lusk, E. Users guide for mpich, a portable implementation of mpi. Tech. rep., Argonne National Lab., IL (United States), 1996. 49,105

[73] Grosse, F., Greenwood, N., Kreus, M., Lenhart, H., Machoczek, D., Pätsch, J., SAlt, L. A., And Thomas, H. Looking beyond stratification: A model-based analysis of the biological drivers of oxygen depletion in the North Sea. Biogeosciences Discussions (2015), 2511-2535. 47

[74] Habib, S., Morozov, V., Finkel, H., et Al. The universe at extreme scale: multi-petaflop sky simulation on the BG/Q. In Proceedings of the International Conference on High Performance Computing, Networking, Storage and Analysis (2012), IEEE Computer Society Press, p. 4. 124

[75] Haerder, T., And Reuter, A. Principles of transaction-oriented database recovery. ACM Computing Surveys (CSUR) 15, 4 (1983), 287-317. 36

[76] Han, J., Hainong, E., Le, G., And Du, J. Survey on nosql database. In Pervasive computing and applications (ICPCA), 2011 6th international conference on (2011), IEEE, pp. 363-366. 23

[77] Harms, K., Leggett, T., Allen, B., Coghlan, S., Fahey, M., Holohan, C., McPheeters, G., And Rich, P. Theta: Rapid installation and acceptance of an XC40 KNL system. Concurrency and Computation: Practice and Experience 30, 1 (2018). 103,106

[78] Hayashibara, N., Defago, X., Yared, R., and Katayama, T. The $\phi$ accrual failure detector. In Reliable Distributed Systems, 2004. Proceedings of the 23rd IEEE International Symposium on (2004), IEEE, pp. 66-78. 83 
[79] Herlihy, M. P., And Wing, J. M. Axioms for concurrent objects. In Proceedings of the 14th ACM SIGACT-SIGPLAN symposium on Principles of programming languages (1987), ACM, pp. 13-26. 33

[80] Hunt, P., Konar, M., Junqueira, F. P., And Reed, B. Zookeeper: Waitfree coordination for internet-scale systems. In USENIX annual technical conference (2010), vol. 8, Boston, MA, USA. 33, 61, 96, 110

[81] Hutto, P. W., and Ahamad, M. Slow memory: Weakening consistency to enhance concurrency in distributed shared memories. In Distributed Computing Systems, 1990. Proceedings., 10th International Conference on (1990), IEEE, pp. 302-309. 33

[82] Jayalakshmi, D., Ranjana, T. R., and Ramaswamy, S. Dynamic data replication across geo-distributed cloud data centres. In International Conference on Distributed Computing and Internet Technology (2016), Springer, pp. 182-187. 129

[83] Karger, D., Lehman, E., Leighton, T., Panigrahy, R., Levine, M., And LEwIN, D. Consistent hashing and random trees: Distributed caching protocols for relieving hot spots on the world wide web. In Proceedings of the twenty-ninth annual ACM symposium on Theory of computing (1997), ACM, pp. 654-663. 63

[84] Karmarkar, N., And Karp, R. M. The difierencing method of set partitioning. Tech. rep., Technical Report UCB/CSD 82/113, Computer Science Division, University of California, Berkeley, 1982. 26

[85] Kelly, N. Node.js asynchronous compute-bound multithreading. 88

[86] Klophaus, R. Riak core: building distributed applications without shared state. In ACM SIGPLAN Commercial Users of Functional Programming (2010), ACM, p. 14. 16, 22, 33, 62, 63

[87] Ko, S., Zhao, J., Xia, J., Afzal, S., Wang, X., Abram, G., Elmqvist, N., Kne, L., Riper, D. V., Gaither, K., Kennedy, S., Tolone, W., RibARSKY, W., AND EBERT, D. S. Vasa: Interactive computational steering of large asynchronous simulation pipelines for societal infrastructure. IEEE Transactions on Visualization and Computer Graphics 20, 12 (Dec 2014), 1853-1862. 104 
[88] Kranzlmüller, D., Kacsuk, P., and Dongarra, J. Recent advances in parallel virtual machine and message passing interface. The International Journal of High Performance Computing Applications 19, 2 (2005), 99-101. 33

[89] Kreps, J., Narkhede, N., RaO, J., et Al. Kafka: A distributed messaging system for log processing. In Proceedings of the NetDB (2011), pp. 1-7. 110

[90] Kunn, M. A semantics-aware I/O interface for high performance computing. In International Supercomputing Conference (2013), Springer, pp. 408-421. 21

[91] Laitala, J. Metadata management in distributed file systems. 128

[92] Lakshman, A., And Malik, P. Cassandra: a decentralized structured storage system. ACM SIGOPS Operating Systems Review 44, 2 (2010), 35-40. 2, 16, 22. 25,33

[93] Lamport, L. How to make a multiprocessor computer that correctly executes multiprocess programs. IEEE transactions on computers, 9 (1979), 690-691. 33

[94] Lamport, L. Fast paxos. Distributed Computing 19, 2 (2006), 79-103. 39

[95] Lamport, L., ET AL. Paxos made simple. ACM Sigact News 32, 4 (2001), 18-25. 69, 64,67

[96] Lamport, L., And Massa, M. Cheap paxos. In Dependable Systems and Networks, 2004 International Conference on (2004), IEEE, pp. 307-314. 39

[97] Latham, R., Ross, R., and Thakur, R. The impact of file systems on mpiio scalability. In European Parallel Virtual Machine/Message Passing Interface Users' Group Meeting (2004), Springer, pp. 87-96. 45

[98] Leach, P. J., AND NaIK, D. A common internet file system (CIFS/1.0) protocol. Tech. rep., Internet-Draft, IETF, 1997. 20

[99] Leavitt, N. Will nosql databases live up to their promise? Computer 43, 2 (2010). 22

[100] Lefevre, C. The cern accelerator complex. Tech. rep., 2008. 93,94 
[101] Legrand, I., Newman, H., Voicu, R., Cirstoiu, C., Grigoras, C., Dobre, C., Muraru, A., Costan, A., Dediu, M., and Stratan, C. MonAlISA: An agent based, dynamic service system to monitor, control and optimize distributed systems. Computer Physics Communications 180, 12 (2009), 24722498. 5,93

[102] Legrand, I., Voicu, R., Cirstoiu, C., Grigoras, C., Betev, L., And Costan, A. Monitoring and control of large systems with MonALISA. Communications of the ACM 52, 9 (2009), 49-55. 5.93

[103] Li, M., Tan, J., Wang, Y., Zhang, L., And Salapura, V. Sparkbench: a comprehensive benchmarking suite for in memory data analytic platform spark. In Proceedings of the 12th ACM International Conference on Computing Frontiers (2015), ACM, p. 53. 46, 47, 135,138

[104] Li, S., Lim, H., Lee, V. W., Ahn, J. H., Kalia, A., Kaminsky, M., AnDersen, D. G., Seongil, O., Lee, S., and Dubey, P. Architecting to achieve a billion requests per second throughput on a single key-value store server platform. In ACM SIGARCH Computer Architecture News (2015), vol. 43, ACM, pp. $476-488.30$

[105] Liu, N., Cope, J., Carns, P., Carothers, C., Ross, R., Grider, G., Crume, A., and Maltzahn, C. On the role of burst buffers in leadership-class storage systems. In Mass Storage Systems and Technologies (MSST), 2012 IEEE 28th Symposium on (2012), IEEE, pp. 1-11. 14

[106] Lloyd, W., Freedman, M. J., Kaminsky, M., and Andersen, D. G. Don't settle for eventual: scalable causal consistency for wide-area storage with cops. In Proceedings of the Twenty-Third ACM Symposium on Operating Systems Principles (2011), ACM, pp. 401-416. 33

[107] Logan, J., And Dickens, P. Towards an understanding of the performance of mpi-io in lustre file systems. In 2008 IEEE International Conference on Cluster Computing (Sept 2008), pp. 330-335. 106

[108] Lorkowski, I., P̈̈тsch, J., Moll, A., And Kühn, W. Interannual variability of carbon fluxes in the North Sea from 1970 to 2006-competing effects of abiotic 
and biotic drivers on the gas-exchange of $\mathrm{CO}_{2}$. Estuarine, Coastal and Shelf Science 100 (2012), 38-57. 47

[109] Mahgoub, A., Ganesh, S., Meyer, F., Grama, A., and Chaterui, S. Suitability of nosql systems - cassandra and scylladb - for iot workloads. In Communication Systems and Networks (COMSNETS), 2017 9th International Conference on (2017), IEEE, pp. 476-479. 26

[110] Matri, P., Alforov, Y., Brandon, A., Kuhn, M., Carns, P., And LudWIG, T. Could blobs fuel storage-based convergence between HPC and Big Data? In Cluster Computing (Cluster), 2017 IEEE International Conference on (2017), IEEE, pp. 81-86. 4, 6

[111] Matri, P., Carns, P., Ross, R., Costan, A., Pérez, M. S., and Antoniu, G. SLoG: A large-scale logging middleware for HPC and Big Data convergence. In Distributed Computing Systems (ICDCS), 2018 IEEE 38th International Conference on (2018), IEEE. 5, 6

[112] Matri, P., Costan, A., Antoniu, G., Montes, J., and Pérez, M. S. Towards efficient location and placement of dynamic replicas for geo-distributed data stores. In Proceedings of the ACM 7th Workshop on Scientific Cloud Computing (2016), ACM, pp. 3-9. 5, 6, 129

[113] Matri, P., Costan, A., Antoniu, G., Montes, J., And Pérez, M. S. Týr: blob storage meets built-in transactions. In High Performance Computing, Networking, Storage and Analysis, SC16: International Conference for (2016), IEEE, pp. 573-584. 4, 5, 6

[114] Matri, P., Costan, A., Antoniu, G., Montes, J., and Pérez, M. S. Týr: Efficient Transactional Storage for Data-Intensive Applications. Technical Report RT-0473, Inria Rennes Bretagne-Atlantique ; Universidad Politécnica de Madrid, Jan. 2016. 4, 5, 6

[115] Matri, P., Costan, A., Antoniu, G., And PÉrez, M. S. TýrFS: Increasing small files access performance with dynamic metadata replication. In Cluster, Cloud and Grid Computing (CCGrid), IEEE/ACM 18th International Symposium on (2018), IEEE. 5.6 
[116] Matri, P., Pérez, M. S., Costan, A., Bougé, L., and Antoniu, G. Keeping up with storage: Decentralized, write-enabled dynamic geo-replication. Future Generation Computer Systems (2017). 5, 6, 129

[117] McLay, R., James, D., Liu, S., Cazes, J., and Barth, W. A user-friendly approach for tuning parallel file operations. In SC14: International Conference for High Performance Computing, Networking, Storage and Analysis (Nov 2014), pp. 229-236. 106

[118] Mehrotra, P., Djomehri, J., Heistand, S., Hood, R., Jin, H., Lazanoff, A., SAini, S., AND Biswas, R. Performance evaluation of amazon ec2 for nasa hpc applications. In Proceedings of the 3rd workshop on Scientific Cloud Computing (2012), ACM, pp. 41-50. 1

[119] Menascé, D. A., And Nakanishi, T. Optimistic versus pessimistic concurrency control mechanisms in database management systems. Information systems 7, 1 (1982), 13-27. 37

[120] Metwally, A., Agrawal, D., And El Abbadi, A. Efficient computation of frequent and top-k elements in data streams. In International Conference on Database Theory (2005), Springer, pp. 398-412. 132

[121] Mishra, V. Titan graph databases with cassandra. In Beginning Apache Cassandra Development. Springer, 2014, pp. 123-151. 26

[122] Momjian, B. PostgreSQL: introduction and concepts, vol. 192. Addison-Wesley New York, 2001. 94

[123] Moore, M., Bonnie, D., Ligon, B., Marshall, M., Ligon, W., Mills, N., Quarles, E., Sampson, S., Yang, S., And Wilson, B. Orangefs: Advancing pvfs. FAST poster session (2011). 124, 127

[124] Mosberger, D. Memory consistency models. ACM SIGOPS Operating Systems Review 27, 1 (1993), 18-26. 32

[125] Mu, S., Cui, Y., Zhang, Y., Lloyd, W., And Li, J. Extracting more concurrency from distributed transactions. In OSDI (2014), vol. 14, pp. 479-494. 67 
[126] Namiot, D. Time series databases. In $D A M D I D / R C D L$ (2015), pp. 132-137. 94

[127] Nicolae, B., Antoniu, G., Bougé, L., Moise, D., and Carpen-Amarie, A. Blobseer: Next-generation data management for large scale infrastructures. Journal of Parallel and distributed computing 71, 2 (2011), 169-184. 28, 52, 62. 95

[128] Ongaro, D., And Ousterhout, J. K. In search of an understandable consensus algorithm. In USENIX Annual Technical Conference (2014), pp. 305-319. 39

[129] Patterson, D. A., Gibson, G., And Katz, R. H. A case for redundant arrays of inexpensive disks (RAID), vol. 17. ACM, 1988. 19

[130] Paz, J. R. G. Introduction to Azure Cosmos DB. In Microsoft Azure Cosmos DB Revealed. Springer, 2018, pp. 1-23. 25

[131] Pritchett, D. BASE: An ACID alternative. Queue 6, 3 (2008), 48-55. 37

[132] RajasekARAn, S., AND REIF, J. Handbook of parallel computing: models, algorithms and applications. CRC Press, 2007. 45

[133] Ramanathan, S., Goel, S., and Alagumalai, S. Comparison of cloud database: Amazon's SimpleDB and Google's Bigtable. In Recent Trends in Information Systems (ReTIS), 2011 International Conference on (2011), IEEE, pp. $165-168.25$

[134] Reed, D. A., And Dongarra, J. Exascale computing and big data. Communications of the ACM 58, 7 (2015), 56-68. 16

[135] ReED, D. P. Implementing atomic actions on decentralized data. ACM Transactions on Computer Systems (TOCS) 1, 1 (1983), 3-23. 68

[136] Ross, R., Carns, P., And Metheny, D. Parallel file systems. In Data Engineering. Springer, 2009, pp. 143-168. 21 
[137] Ross, R. B., Thakur, R., et AL. PVFS: A parallel file system for linux clusters. In Proceedings of the 4th annual Linux showcase and conference (2000), pp. 391-430. 2, 19, 20, 21, 33

[138] Sakr, S., Liu, A., Batista, D. M., And Alomari, M. A survey of large scale data management approaches in cloud environments. IEEE Communications Surveys \& Tutorials 13, 3 (2011), 311-336. 27

[139] Sandberg, R., Goldberg, D., Kleiman, S., Walsh, D., and Lyon, B. Design and implementation of the Sun network filesystem. In Proceedings of the Summer USENIX conference (1985), pp. 119-130. 20

[140] Schmuck, F. B., And Haskin, R. L. GPFS: A shared-disk file system for large computing clusters. In FAST (2002). 2, 20, 33, 103,107

[141] Schütt, T., Schintke, F., And Reinefeld, A. Scalaris: reliable transactional p2p key/value store. In Proceedings of the 7th ACM SIGPLAN workshop on ERLANG (2008), ACM, pp. 41-48. 36

[142] Schwan, P., ET AL. Lustre: Building a file system for 1000-node clusters. In Proceedings of the 2003 Linux symposium (2003), vol. 2003, pp. 380-386. 2, 19 , 20, 28, 33, 47, 52, 103, 107, 124, 126, 134

[143] Sen, R., Farris, A., And Guerra, P. Benchmarking apache accumulo bigdata distributed table store using its continuous test suite. In Big Data (BigData Congress), 2013 IEEE International Congress on (2013), IEEE, pp. 334-341. 26

[144] Shipman, G. M., Dillow, D. A., Oral, H. S., and Wang, F. The spider center wide file system; from concept to reality. Tech. rep., Oak Ridge National Lab.(ORNL), Oak Ridge, TN (United States); Center for Computational Sciences, 2009. 54

[145] Shvachko, K., Kuang, H., Radia, S., And Chansler, R. The hadoop distributed file system. In Mass storage systems and technologies (MSST), 2010 IEEE 26th symposium on (2010), Ieee, pp. 1-10. 15, 19, 20, 46, 52, 96

[146] Sigoure, B. Opentsdb: The distributed, scalable time series database. Proc. OSCON 11 (2010). 94 
[147] Skeen, D., And Stonebraker, M. A formal model of crash recovery in a distributed system. IEEE Transactions on Software Engineering, 3 (1983), 219228. 39

[148] Srinivasan, V., Bulkowski, B., Chu, W.-L., Sayyaparaju, S., Gooding, A., Iyer, R., Shinde, A., And Lopatic, T. Aerospike: architecture of a real-time operational DBMS. Proceedings of the VLDB Endowment 9, 13 (2016), 1389-1400. 2, 16, 22, 62

[149] Stegert, C. Modelling the annual development of a zooplankton population in the north sea. 47

[150] Stegert, C., Moll, A., and Kreus, M. Validation of the three-dimensional ecoham model in the german bight for 2004 including population dynamics of pseudocalanus elongatus. Journal of Sea Research 62, 1 (2009), 1-15. 47

[151] Stoica, I., Morris, R., Liben-Nowell, D., Karger, D. R., Kaashoek, M. F., Dabek, F., And Balakrishnan, H. Chord: a scalable peer-to-peer lookup protocol for internet applications. IEEE/ACM Transactions on Networking (TON) 11, 1 (2003), 17-32. 63

[152] Stone, J. An efficient library for parallel ray tracing and animation. Tech. rep., Intel Supercomputer Users Group Proceedings, 1995. 47

[153] Szeredi, M. FUSE: Filesystem in userspace. http://fuse.sourceforge.net/, 2010. Accessed: 2017-11-01. 53

[154] Terry, D. B., Theimer, M. M., Petersen, K., Demers, A. J., Spreitzer, M. J., AND HaUser, C. H. Managing update conflicts in Bayou, a weakly connected replicated storage system, vol. 29. ACM, 1995. 68

[155] Tesorieno, C. Getting Started with OrientDB. Packt Publishing Ltd, 2013. 26

[156] Tessier, F., Vishwanath, V., and Jeannot, E. Tapioca: An i/o library for optimized topology-aware data aggregation on large-scale supercomputers. In 2017 IEEE International Conference on Cluster Computing (CLUSTER) (Sept 2017), pp. 70-80. 106 
[157] Thakur, R., Gropp, W., And Lusk, E. Data sieving and collective i/o in romio. In Frontiers of Massively Parallel Computation, 1999. Frontiers' 99. The Seventh Symposium on the (1999), IEEE, pp. 182-189. 35

[158] Thanh, T. D., Mohan, S., Choi, E., Kim, S., and Kim, P. A taxonomy and survey on distributed file systems. In Networked Computing and Advanced Information Management, 2008. NCM'08. Fourth International Conference on (2008), vol. 1, IEEE, pp. 144-149. 19

[159] The Apache Foundation. Apache Cassandra. https://cassandra.apache. org/, 2014. Accessed: 2017-11-20. 25

[160] Thomas, R. H. A majority consensus approach to concurrency control for multiple copy databases. ACM Transactions on Database Systems (TODS) 4, 2 (1979), 180-209. 36

[161] Tiepolo, G. Getting started with rethinkdb. Packt Publishing Ltd, 2016. 25

[162] Tsujita, Y., Yoshinaga, K., Hori, A., Sato, M., Namiki, M., And IsHIKAWA, Y. Multithreaded two-phase i/o: Improving collective mpi-io performance on a lustre file system. In 2014 22nd Euromicro International Conference on Parallel, Distributed, and Network-Based Processing (Feb 2014), pp. 232-235. 106

[163] Vilayannur, M., Lang, S., Ross, R., Klundt, R., Ward, L., et al. Extending the POSIX I/O interface: a parallel file system perspective. Tech. rep., Argonne National Laboratory (ANL), 2008. 21

[164] Vora, M. N. Hadoop-hbase for large-scale data. In Computer science and network technology (ICCSNT), 2011 international conference on (2011), vol. 1, IEEE, pp. 601-605. 46, 126

[165] Vukotic, A., Watt, N., Abedrabbo, T., Fox, D., and Partner, J. Neo4j in action. Manning Publications Co., 2014. 26

[166] Wang, L., Zhan, J., Luo, C., Zhu, Y., Yang, Q., He, Y., GaO, W., Jia, Z., Shi, Y., Zhang, S., Zheng, C., Lu, G., Zhan, K., Li, X., And QıU, B. BigDataBench: A big data benchmark suite from Internet services. 
In 2014 IEEE 20th International Symposium on High Performance Computer Architecture (HPCA) (feb 2014), IEEE. 46, 47

[167] Weil, S. A., Brandt, S. A., Miller, E. L., Long, D. D., and Maltzahn, C. Ceph: A scalable, high-performance distributed file system. In Proceedings of the 7th symposium on Operating systems design and implementation (2006), USENIX Association, pp. 307-320. 2, 16, 20, 27, 28, 31, 52, 56, 106, 123, 128, 134

[168] Weil, S. A., Brandt, S. A., Miller, E. L., and Maltzahn, C. Crush: Controlled, scalable, decentralized placement of replicated data. In Proceedings of the 2006 ACM/IEEE conference on Supercomputing (2006), ACM, p. 122. 56

[169] Weil, S. A., Leung, A. W., Brandt, S. A., and Maltzahn, C. Rados: a scalable, reliable storage service for petabyte-scale storage clusters. In Proceedings of the 2nd international workshop on Petascale data storage: held in conjunction with Supercomputing'07 (2007), ACM, pp. 35-44. 52, 62, 95, 123

[170] Yang, S., Yan, X., Zong, B., And Khan, A. Towards effective partition management for large graphs. In Proceedings of the 2012 ACM SIGMOD International Conference on Management of Data (2012), ACM, pp. 517-528. 26

[171] Ye, J., McGinnis, S., And Madden, T. L. BLAST: improvements for better sequence analysis. Nucleic acids research 34, suppl_2 (2006), W6-W9. 47

[172] Yi, H., Rasquin, M., FAng, J., And Bolotnov, I. A. In-situ visualization and computational steering for large-scale simulation of turbulent flows in complex geometries. In 2014 IEEE International Conference on Big Data (Big Data) (Oct 2014), pp. 567-572. 104

[173] Zaharia, M., Chowdhury, M., Das, T., Dave, A., Ma, J., McCauley, M., Franklin, M. J., Shenker, S., And Stoica, I. Resilient distributed datasets: A fault-tolerant abstraction for in-memory cluster computing. In Proceedings of the 9th USENIX conference on Networked Systems Design and Implementation (2012), USENIX Association, pp. 2-2. 46, 47, 135 
[174] Zhang, Y., Power, R., Zhou, S., Sovran, Y., Aguilera, M. K., And Li, J. Transaction chains: achieving serializability with low latency in geo-distributed storage systems. In Proceedings of the Twenty-Fourth ACM Symposium on Operating Systems Principles (2013), ACM, pp. 276-291. 39

[175] Zheng, Q., Ren, K., Gibson, G., Settlemyer, B. W., and Grider, G. DeltaFS: Exascale file systems scale better without dedicated servers. In Proceedings of the 10th Parallel Data Storage Workshop (2015), ACM, pp. 1-6. 2

[176] Ziegeler, S., Atkins, C., Bauer, A., And Pettey, L. In situ analysis as a parallel i/o problem. In Proceedings of the First Workshop on In Situ Infrastructures for Enabling Extreme-Scale Analysis and Visualization (New York, NY, USA, 2015), ISAV2015, ACM, pp. 13-18. 104 